\title{
Ref-1 Redox Activity Alters Cancer Cell Metabolism in Pancreatic Cancer: Exploiting This Novel Finding as a Potential Target
}

\section{Silpa Gampala}

Indiana University School of Medicine

\section{Fenil Shah}

Indiana University School of Medicine

\section{Xiaoyu Lu}

Indiana University School of Medicine

Hye-ran Moon

Purdue University

\section{George Sandusky}

Indiana University School of Medicine

\section{Emily Hulsey}

Indiana University School of Medicine

\section{Amber Mosley}

Indiana University School of Medicine

\section{Bumsoo Han}

Purdue University

Mircea Ivan

Indiana University School of Medicine

Mark R Kelley

Indiana University School of Medicine

\section{Chi Zhang}

Indiana University School of Medicine

Melissa L Fishel (D mfishel@iu.edu )

Indiana University https://orcid.org/0000-0002-9436-6382

\section{Research}

Keywords: scRNA-seq, Ref-1, Redox function, Metabolism, OXPHOS, Cancer associated fibroblasts (CAFs), pancreatic cancer, mitochondria

Posted Date: December 15th, 2020 
DOl: https://doi.org/10.21203/rs.3.rs-125304/v1

License: (c) (1) This work is licensed under a Creative Commons Attribution 4.0 International License. Read Full License

Version of Record: A version of this preprint was published at Journal of Experimental \& Clinical Cancer Research on August 10th, 2021. See the published version at https://doi.org/10.1186/s13046-021-02046$\mathrm{x}$. 


\section{Abstract}

Background: Pancreatic cancer is a complex disease with a desmoplastic stroma, extreme hypoxia, and inherent resistance to therapy. Understanding the signaling and adaptive response of such an aggressive cancer is key to making advances in therapeutic efficacy. Redox factor-1 (Ref-1), a redox signaling protein, regulates the DNA binding activity of several transcription factors (TFs), including HIF-1a, STAT3 and NFKB . The conversion of these TFs from an oxidized to reduced state lead to enhancement of their DNA binding. In our previously published work, knockdown of Ref-1 under normoxia resulted in altered gene expression patterns on pathways including EIF2, protein kinase A, and mTOR. In this study, single cell RNA sequencing (scRNA-seq) and proteomics were used to explore the effects of Ref-1 on metabolic pathways under hypoxia.

Methods: scRNA-seq comparing pancreatic cancer cells expressing less than $20 \%$ of the Ref- 1 protein was analyzed using left truncated mixture Gaussian model and validated using proteomics and qRT-PCR. The impact of knocking down Ref-1 under hypoxia was dramatic on the mitochondria and metabolic pathways. Ref-1's role in mitochondrial function was confirmed using mitochondrial function assays and qRT-PCR. Further, the effect of Ref-1 redox function inhibition against pancreatic cancer metabolism was assayed using 3D co-culture in vitro and xenograft studies in vivo and compared to Devimistat, a drug that disrupts mitochondrial metabolism.

Results: Distinct transcriptional variation in central metabolism, cell cycle, apoptosis, immune response, and genes downstream of a series of signaling pathways and transcriptional regulatory factors were identified in Ref-1 knockdown vs Scrambled control from the scRNA-seq data. Mitochondrial DEG subsets downregulated with Ref-1 knockdown were significantly reduced following Ref-1 redox inhibition and more dramatically in combination with Devimistat in vitro. Mitochondrial function assays demonstrated that Ref-1 knockdown and Ref-1 redox signaling inhibition decreased utilization of TCA cycle substrates and slowed the growth of pancreatic cancer co-culture spheroids. In vivo xenograft studies demonstrated that tumor reduction was potent with Ref-1 redox inhibitor similar to Devimistat.

Conclusion: Ref-1 redox signaling inhibition conclusively alters cancer cell metabolism by causing TCA cycle dysfunction while also reducing the pancreatic tumor growth in vitro as well as in vivo.

\section{Background}

Pancreatic Ductal Adenocarcinoma (PDAC) is a devastating disease that has a five-year survival rate of $\sim 10 \% .{ }^{1}$ Associated with the actual tumor epithelial cell is a complex stroma composed of cancerassociated fibroblasts (CAFs), immune and endothelial cells, and a rich extracellular matrix (ECM) ${ }^{2,3}$ It is predominantly characterized by severe hypoxia, a complex tumor micro-environment (TME) and altered metabolism that favors anabolic mechanisms. ${ }^{4-6}$ PDAC cells exhibit altered cell metabolism to meet their bioenergetic, biosynthetic and reduction-oxidation (redox) needs. ${ }^{7}$ For example, one of the above mentioned anabolic pathways is pentose phosphate pathway, which supplies intermediates for 
nucleotide biosynthesis. ${ }^{8}$ Other biomolecules like glutamine and aspartate which are necessary building blocks for tumor biomass are accumulated from the byproducts of the tricarboxylic acid (TCA) cycle. ${ }^{9,10}$

The regulation of many tumor suppressor genes and oncogenes is rewired to facilitate these new demands of the growing tumor. Genes such as TP53, KRAS and $M Y C^{8}$ can all be involved in the rewiring of the tumor's metabolic capacity. Smolkova et al., demonstrated four 'waves' of gene expression changes during carcinogenesis that involve reversible shifting between glycolysis and OXPHOS (oxidative phosphorylation) with the assistance of oncogenic signaling and mitochondrial gene reprogramming. These changes are the major alterations responsible for tumor initiation and metastasis. ${ }^{11}$ The majority of PDAC cells harbor KRAS mutations which further enable tumor cells to survive under stress conditions like the lack of oxygen through stabilization of Hypoxia Inducible Factors (HIFs). Stabilized HIFs shift the balance between glycolysis and OXPHOS further away from OXPHOS towards glycolysis, partly by decreasing the activity of pyruvate dehydrogenase $(\mathrm{PDH}) .{ }^{12-14}$

Redox factor 1/Apurinic-apyrimidinic endonuclease 1 (Ref-1/APE1) (henceforth referred to as Ref-1) is a protein potentially impacting mitochondrial function through two of its major functions. Ref-1's function as an endonuclease in base excision repair (BER) pathway could impact mitochondria as it repairs damaged mitochondrial DNA. ${ }^{15-18}$ Ref-1 also possesses redox signaling promoting the binding of transcription factors (TFs) such as HIF-1a, STAT3, NF-kB, and AP-1, among others to DNA. ${ }^{19-24}$ Ref-1's regulation of HIF-1a along with other TFs may contribute to the metabolic rewiring that is observed in many cancers. ${ }^{24,25}$ As HIF stabilization would shift the cells toward glycolysis and away from oxygenrequiring OXPHOS, blockade of Ref-1 under hypoxia leads to a downregulation of glycolysis as HIF1 cannot be fully activated when Ref- 1 is blocked. Surprisingly, we also observed dramatic gene expression changes in the TCA cycle genes as well as genes within the complexes of the ETC. There are reports in cancer including PDAC that depending on the glucose and oxygen conditions that OXPHOS can be utilitized especially under extreme oxygen conditions. ${ }^{26,27}$ These effects can be driven by ROS and even Akt and NFkB signaling pathways and impact upon metabolism. ${ }^{28}$ Multiple cancers including PDAC exhibit increased Ref-1 expression levels that concomitantly associate with their resistance to radiation and chemotherapy and potentially to a metabolic shift, leading to poorer patient prognosis. ${ }^{29,30}$

Due to Ref-1's role in regulating multiple TFs associated with cancer-related pathways, Ref- 1 has become a novel target for anti-cancer therapy in PDAC. ${ }^{25,31,32}$ Although the redox signaling functions of Ref-1 have been studied in a number of biological contexts, its interacting TF partner(s) critical for regulation of metabolic pathways in PDAC is still not well understood. We previously published single-cell RNA Sequencing (scRNA-seq) data in low passage patient-derived PDAC cells following Ref- 1 knockdown under normoxia. ${ }^{25}$ In order to better understand the role of Ref-1 on hypoxia signaling in PDAC, we used scRNA-seq coupled with proteomics to further investigate the role of Ref-1 under hypoxic conditions. In our current study, bioinformatic analysis of hypoxia scRNA-seq data demonstrated that knockdown of Ref-1 led to distinct variation in metabolic pathways including TCA cycle (top downregulated pathway), glycolysis and OXPHOS, HIF regulated genes, apoptosis, immune response, and a series of signaling 
pathways and transcriptional regulatory factors. In addition to the expected decrease in glycolysis, dramatic decreases in gene expression in TCA cycle and complexes within the electron transport chain were also observed. Using Ref-1 siRNA and specific Ref-1 redox inhibitors, we validated these findings using qRT-PCR on a panel of mitochondrial differentially expressed genes (DEGs) and quantitation of mitochondrial substrates. We also extended these studies to cancer-associated fibroblasts (CAFs) which constitute a crucial component of the PDAC TME. In order to ascertain the clinical relevance of our findings, we performed in vivo studies using mice with tumor cells co-injected with CAFs treated with Ref1 inhibitor, APX2009 or the comparator compound, Devimistat, also known as CPI-613. APX2009 is a second-generation Ref-1 redox signaling inhibitor. ${ }^{33-36}$ Devimistat is a first-in-class dehydrogenase inhibitor targeting the TCA cycle. ${ }^{37-39}$ In summary, our findings demonstrate a newly discovered role for Ref- 1 in TCA cycle and expression of genes within the mitochondrial complexes even under hypoxia as well as confirm that Ref-1 redox function has a role in regulating PDAC hypoxia signaling pathways as HIF regulated genes and glycolysis were also affected. Additionally, we identified a potential PDAC therapeutic approach using APX2009 and Devimistat in multiple 3D co-culture models containing both pancreatic cancer cells as well as CAFs which was also validated in vivo.

\section{Methods}

\section{Cell culture}

Pa03C, Panc10.05, and CAF19 cells were obtained from Dr. Anirban Maitra at The Johns Hopkins University ${ }^{40}$ and were then transduced with TdTomato for tumor cells or EGFP for CAFs as previously described. ${ }^{41}$ CAF02-hTERT cells were isolated using the outgrowth method as previously described. ${ }^{42,43}$ Cells were maintained at $37{ }^{\circ} \mathrm{C}$ in $5 \% \mathrm{CO} 2$ and grown in DMEM (Invitrogen; Carlsbad, CA) with $10 \%$ Serum (Hyclone; Logan, UT), or under hypoxic conditions of $1 \% \mathrm{O}_{2} / 5 \% \mathrm{CO}_{2}$ using a Ruskinn Invivo 200 hypoxia work station. Cell line identity was confirmed by DNA fingerprint analysis (IDEXX BioResearch, Columbia, MO) for species and baseline short-tandem repeat analysis testing. Cell lines were $100 \%$ human and a nine-marker short tandem repeat analysis exists on file. They were also confirmed to be mycoplasma free.

\section{Single cell RNA sequencing (scRNA-seq)}

scRNA-seq was performed as previously reported. ${ }^{25}$ Libraries were prepared by The Purdue Genomics Facility (Purdue University, West Lafayette, IN) using a Nextera kit (Illumina, San Diego, CA, USA). Unstranded $2 \times 100$ bp reads were sequenced using the HiSeq2500 (Illumina, San Diego, CA, USA) on rapid run mode in one lane.

\section{scRNA-seq Data Analysis}

FastQC was applied to evaluate the quality of the single cell RNA sequencing data. Counts were called for each cell sample by using STAR alignment pipeline against human GRCh38 reference genome. Cells with less than 250 or more than 10,000 non-zero expressed genes were excluded from the analysis. Cells with 
more than $15 \%$ counts mapped to the mitochondrial genome were excluded as low quality cells, resulting in 40 Ref-1 knockdown (KD) and 48 Scr cells under hypoxia condition and 27 Ref- 1 KD and 46 Scr cells under normoxia condition for further analysis.

Unsupervised cell clustering was performed using Seurat v3.0 with the variedly expressed genes identified by default parameters. Cell clusters were annotated by the experimental condition and their uniquely highly expressed genes. Differentially expressed genes and gene expression states were identified by using the left truncated mixture Gaussian model based test, with FDR $<0.05$ as the significant cutoff. ${ }^{44}$ Gene co-regulation modules were identified by using our inhouse developed Boolean matrix decomposition method MEBF. ${ }^{45}$ Pathway enrichment of the differentially expressed genes and the genes in each co-regulation module were analyzed by a hypergeometric test against the canonical gene sets and transcriptional regulatory factor targets retrieved from MsigDB v6 ${ }^{46}$, with $p<0.001$ as the significant cutoff.

\section{Quantitative global proteomic comparison of protein levels}

Sample preparation, mass spectrometry analysis, bioinformatics and data evaluation were performed in collaboration with the Proteomics Core Facility (Indiana University School of Medicine, Indianapolis, IN).

Detailed method can be found in supplementary information and was adapted from literature reports ${ }^{47,48}$ and vendor provided protocols. Briefly, cells were lysed using urea lysis buffer. Protein isolated and trypsin/Lys-C digested for peptides. Peptides were fractionated using Pierce ${ }^{\mathrm{TM}} \mathrm{High} \mathrm{pH}$ reversed-phase peptide fractionation spin columns and then subjected to Nano-LC-MS/MS analysis.

\section{Transfection with Ref-1 and Scrambled siRNA}

The siRNAs used were: Scrambled (Scr) (5'-CCAUGAGGUCAGCAUGGUCUG-3', 5'GACCAUGCUGACCUCAUGGAA-3') and siRef-1 (5'-GUCUGGUACGACUGGAGUACC-3', 5'UACUCCAGUCGUACCAGACCU-3'). All siRNA transfections were performed as previously

described. ${ }^{41,49-52}$ Briefly, $1 \times 10^{5}$ cells are plated per well of a 6-well plate and allowed to attach overnight. The next day, Lipofectamine RNAiMAX reagent (Invitrogen, Carlsbad, CA) was used to transfect in the Ref1 and Scr siRNA at concentrations of $10 \mathrm{nM}$ following the manufacturer's indicated protocol. Opti-MEM, siRNA, and Lipofectamine was left on the cells for $16 \mathrm{~h}$ and then regular DMEM media with $10 \%$ Serum was added. Cells were assayed for RNA and protein expression 3 days following transfection.

\section{Inhibitor treatment}

APX3330 and APX2009 were provided by Apexian Pharmaceuticals (20 N. Meridian, Indianapolis, IN 46204, USA) and prepared as previously described. ${ }^{35,36,53}$ Devimistat (CPI-613) was purchased from APExBio (Boston, MA) and reconstituted in $100 \%$ DMSO as a $50 \mathrm{mM}$ stock for in vitro drug treatments. Both agents are in clinical trials (clinicaltrials.gov; NCT03375086 and NCT03435289 respectively).

Pa03C cells $\left(2 \times 10^{5}\right)$ were plated into 6-well plates and allowed to attach overnight. The following day, APX3330, APX2009, or Devimistat in low serum (5\%) DMEM media were added to the wells. DMSO was 
used as the vehicle control. Cells were treated for 24 hours, after which they were collected for analysis.

\section{Mitochondrial Function Assay}

S-1 Mitoplates (Biolog, Hayward, CA) were used to investigate mitochondrial function. Assays were performed as per manufacturer's protocol. Briefly, plates were activated by adding the Assay Mix to the wells to dissolve the substrates, for at least 60 minutes at $37^{\circ} \mathrm{C}$. Following siRef- 1 transfection or drug treatment, cells were collected, counted, resuspended in provided buffer and plated at $5 \times 10^{4}$ cells $/$ well. This resuspension was added to the plate, which was immediately read at $590 \mathrm{~nm}$ kinetically at $5 \mathrm{~min}$ intervals for 4 hours at $37^{\circ} \mathrm{C}$. Data was analyzed using Graphpad Prism 8, and statistical significance was determined using the 2-way ANOVA and p-values $<0.05$ were considered statistically significant.

\section{Western Blot Analysis}

For whole cell lysates, cells were harvested, lysed in RIPA buffer (Santa Cruz Biotechnology, Santa Cruz, $\mathrm{CA}$ ), and protein was quantified and electrophoresed. Immunoblotting was performed using the following antibodies: Ref-1 (1:1000, Novus Biologicals, Littleton, CO) and Vinculin (1:1000, Sigma, St. Louis, MO). For subsequent experiments, Ref- 1 expression needed to be decreased by at least $80 \%$ compared to scrambled control in order to be considered for further analysis.

\section{qRT-PCR}

qRT-PCR was used to measure the mRNA expression levels of the various genes identified from the scRNA-seq analysis. Following transfection, total RNA was extracted from cells using the Qiagen RNeasy Mini kit (Qiagen, Valencia, CA) according to the manufacturer's instructions. First-strand cDNA was obtained from RNA using random hexamers and MultiScribe reverse transcriptase (Applied Biosystems, Foster City, CA). Quantitative PCR was performed using SYBR Green Real Time PCR master mix (Applied Biosystems, Foster City, CA) in a CFX96 Real Time detection system (Bio-Rad, Hercules, CA). The relative quantitative mRNA level was determined using the comparative Ct method using actin as the reference gene. The primers used for qRT-PCR are detailed in Supplement Table 2. Experiments were performed in at least triplicate for each sample. Statistical analysis performed using the $2^{-}{ }^{\Delta} C_{T}$ method and analysis of covariance (ANCOVA) models, as previously published. ${ }^{23}$

In Vivo Studies

All animal studies were conducted under the guidelines of the National Institutes of Health and were approved by the Institutional Animal Care and Use Committee of Indiana University School of Medicine. NOD.Cg-Prkdcscid II2rgtm1 Wjl/SzJ (NOD/SCIDy(-/-))mice (or other strains) were obtained from the In Vivo Therapeutics Core of the Indiana University Simon Cancer Center. Animals were maintained under pathogen-free conditions and a $12 \mathrm{~h}$ light-dark cycle. NOD scid gamma (NOD.Cg-Prkdcsid $/ 12 \mathrm{rg}^{\text {tm }} \mathrm{WWj} / \mathrm{SzJ}$ ) or NSG mice were subcutaneously implanted with $2.5 \times 10^{6} \mathrm{Pa03C}$ cells or with $5 \times 10^{6}+15 \times 10^{6}$ Panc10.05 + CAF19 cells in the hind flank using a $200 \mu \mathrm{l}$ volume of 50:50 solution of Matrigel:DMEM medium. When tumor volumes reached $\sim 100 \mathrm{~mm}^{3}$, the mice were randomized into 4 groups of $8-9$ mice 
before commencing treatment $(\sim 11$ days for Pa03C and $\sim 24$ days for Panc10.05 + CAF19 post implantation). The treatment regimen consisted of oral administration of either vehicle (Propylene Glycol, Kolliphor HS15, Tween 80 (PKT) as previously reported ${ }^{34}$ ) or $35 \mathrm{mg} / \mathrm{kg}$ APX2009 or $50 \mathrm{mg} / \mathrm{kg}$ Devimistat dosed twice a day for 15 (for Pa03C xenografts) or 20 (for Panc10.05 + CAF19 xenografts) days. Tumor volumes were measured twice a week and mice were weighed once a week. Data was analyzed using Graphpad Prism 8. Statistical significance was determined using the one-way ANOVA and p-values $<0.05$ were considered statistically significant.

\section{D Co-Culture Assays}

Ultra-low attachment 96-well plates (Corning Inc., Life Sciences) were used to generate 3-dimensional tumor spheroids in the presence of CAFs as reported in the Results and as described previously. ${ }^{41,54,55}$ Following plating, cells were treated on Days 4,7 , and 10 with media containing $5 \%$ serum, $3 \%$ growth factor reduced Matrigel, and inhibitors as indicated. On Days 4, 7, 10, and 14, spheroids were analyzed using Thermo ArrayScan high-content imaging system. ${ }^{56}$ Images of 3D structures were captured by ArrayScan using a 2.5x objective for TdTomato and EGFP; then 2D projections were processed to quantify differences in total intensity of both CAFs and tumor.

\section{Interstitial tumor-microenvironment-on-chip (iT-MOC) Assay}

The interstitial tumor-microenvironment-on-chip (iT-MOC) is a 3D in vitro microfluidic platform having two layers of microchannels interfaced with a porous membrane in between. Details of fabrication and preparation were described previously. ${ }^{57-59}$ Briefly, pancreatic cancer cells (Panc10.05) and cancerassociated fibroblasts (CAF19) were mixed at a 1:1 cell ratio into the cell-collagen mixture. The initial cell concentration was $2 \times 10^{6}$ cells $/ \mathrm{mL}$ for each cell type. After loading, the devices were incubated at $37^{\circ} \mathrm{C}$ for $1 \mathrm{hr}$ for collagen gelation. Then, the culture medium was perfused by pressurizing the interstitial channel.

In order to assess drug efficacy, the PDAC iT-MOC was cultured for 48 hrs before treatment. On day 2, drugs were perfused through the capillary channel. The drug solutions were prepared as $0 \mu \mathrm{M}$ (control), $30 \mu \mathrm{M}$ of APX2009, $25 \mu \mathrm{M}$ of Devimistat, and the combination of APX2009 $(30 \mu \mathrm{M})$ and Devimistat $(25 \mu \mathrm{M})$ in the culture medium. On day 5 , the channels were washed with drug-free medium and reperfused with the same drug-containing medium. On day 8, the iT-MOC platforms were washed and cultured in normal culture medium for $24 \mathrm{hrs}$ before the viability assay. Drug efficacy was analyzed in two ways, cell growth and cell survival as detailed in the supplementary methods.

\section{Statistics}

All the experiments were performed at least three independent times. The data obtained were expressed as 'Mean + Standard Error'. Significance was calculated as per either 2-way ANOVA or unpaired t-test wherever applicable using Graph Pad Prism Version 8. For iT-MOC assays, Drug efficacy differences of each drug control were statistically analyzed by Tukey post hoc multiple comparison test provided in ANOVA. The difference was considered statistically significant when $p$-value $<0.05$. For qRT-PCR, analysis 
of covariance models was performed to test the Ct difference of each target gene value between treatment with APX2009, Devimistat (CPI-613), and vehicle (DMSO) or siRNA and scrambled control after standardization by reference gene (RPL6/Actin) using ANCOVA as previously described ${ }^{23}$. A p-value of at least $<0.05$ was considered statistically significant.

To test the tumor growth rate following treatment (i.e., the regression slope for a particular treatment) and differences in tumor growth rates between treatments (i.e., the difference in regression slopes between two treatments) in the in vivo tumor model, mixed effect repeated measure regression models with random intercept were used. ${ }^{60}$ Tumor weights over time were estimated and compared between treatments from the regression models. To be considered statistically significant, a p-value of at least 0.05 was used. All statistical analysis was conducted using SAS 9.4 (SAS, Inc., Cary, NC, 2016).

\section{Results}

\section{Transcriptomic variation caused by Ref-1 inhibition under hypoxia}

A scRNA-seq experiment was conducted on a patient-derived pancreatic cancer line, Pa03C under the following conditions: Ref-1 knockdown (siRef-1), Scrambled control (Scr), Hypoxia (H), and Normoxia (N). The Fluidigm C1 Smart Seq2 protocol was used for the scRNA-seq as described in Methods. This platform enabled a saturated measure of the transcriptome in each single cell, resulting in high sensitivity in the identification of the genes, biological pathways or transcriptional regulatory modules regulated by Ref-1. ${ }^{61,62}$ In total, 40 cells transfected with Ref- 1 siRNA and $48 \mathrm{Scr}$ control cells were collected after hypoxia exposure and after removing low quality cells. We combined this dataset with 27 cells of Ref- 1 siRNA and $25 \mathrm{Scr}$ control collected under normoxia from our previous work. In total, 18,204 genes with a significant none zero expression were detected in this data set. It is noteworthy the major goal of this study was not for cell type identification. Instead, we focused on identification of the distinct functional changes that are due to the inhibition of Ref-1 and/or perturbed oxygen level, i.e. biologically explainable gene sets that show distinct expression variation in a subset of the sample. Our previous studies demonstrated these cell numbers provide enough statistical power for the analysis of differentially expressed genes and functional modules. ${ }^{44,63}$

The expression level of Ref-1 (APEX1) in the single cells demonstrated the gene was successfully knocked down in the siRef-1 group (Fig. 1G). Unsupervised cell clustering analysis was first conducted on the cells under all conditions (See Methods). Six cell clusters were identified, which were all highly associated with the experimental groups (Fig. 1A, B). Genes overly expressed in each cluster were identified by using left truncated mixture Gaussian model. The cell clusters were further annotated by the experimental condition and their specifically expressed genes and pathways. Two clusters within the hypoxia Scr control cells were identified. Pathway enrichment analysis of the marker genes of each cluster suggested one group has high expression of HIF-1a-regulated genes while the other has 
specifically elevated lactate production. Within the siRef-1 cells under hypoxia, two clusters were identified; one cluster corresponded to consistently down-regulated genes that were downstream of HIF$1 \mathrm{a}$ which would be expected based on Ref-1 redox regulation of HIF-1a. The other cluster corresponded to genes related to upregulated translation (Fig. 1B).

Figure 1C illustrates the heatmap of the top variably expressed genes in all the tumor cells. Distinct clusters of the cells associated with different conditions were observed. Dendrogram derived by a hierarchical clustering analysis demonstrated dramatic changes in gene expression caused by hypoxia. DEGs between siRef-1 and Scr control were identified using left truncated mixture Gaussian model. ${ }^{44}$ In hypoxia vs normoxia cells, we have identified 3,521 upregulated and 472 downregulated genes $(F D R<$ 0.05). Similarly, there are significant changes in gene expression upon comparison of siRef- 1 and scrambled control under the hypoxia condition, where 386 upregulated and 1,251 downregulated genes were identified. Matched samples were also collected for bulk proteomic analysis of the four conditions, totaling 6,931 proteins measured. Proteomic analysis confirmed significant differences between hypoxia and normoxia (2,155 upregulated and 1,825 downregulated proteins, $p<0.01$ by Mann Whitney test) as well as siRef- 1 and scrambled control under hypoxia condition (513 upregulated and 390 downregulated proteins, $p<0.01$ by Mann Whitney test). Complete lists of the dysregulated genes and proteins were provided in Supplementary Tables S3.

To characterize the Ref-1-regulated genes under hypoxia, we focused on the 386 upregulated and 1,251 downregulated genes in siRef- 1 vs Scr control under $1 \% \mathrm{O}_{2}$ levels. Pathway analysis suggested the upregulated genes significantly $(p<0.001)$ enrich mRNA processing and transcription, cell cycle, DNA replication, MYC signaling, HIV infection, and protein metabolism. PDAC cells with lower Ref- 1 expression proliferate much slower compared to Scr control and the cell cycle genes that were upregulated were mainly nucleoporins and some proteosome genes, but not cyclins. The downregulated genes mainly $(\mathrm{p}<$ 0.001) enrich in central metabolic pathways (glycolysis, TCA cycle, pentose phosphate, oxidative phosphorylation, amino acids, protein and lipid metabolism), with HIF-1a and PDGF signaling pathways also affected (Fig. 1D). For comparison under normoxia, there were also more genes downregulated than upregulated identified in siRef-1 vs Scr control (447 vs. 120 , FDR < 0.05). Pathway enrichment analysis revealed the upregulated genes significantly $(p<0.001)$ enrich to transcription, HIV infection and immune response pathways, while the downregulated genes significantly $(p<0.001)$ enrich to central metabolic pathways (glycolysis, pentose phosphate, oxidative phosphorylation), immune response (antigen presentation, T cell receptor and IL6 signaling), and multiple signaling pathways (p53, MAPK, MET and HIF-1a). We also integrated the scRNA data analysis with the proteomic analysis and determined that the differentially expressed genes and pathways identified from the scRNA-seq data are highly consistent to the significant proteins observed in the proteomics data, especially for the upregulated cell cycle (nucleoporins) and transcription pathways and downregulated metabolic, apoptosis and signaling pathways under hypoxia condition (Fig. 1D,F). Figure 1G shows selected genes associated with each condition and specific cell clusters within the siRef- 1 hypoxia group. Complete lists of the differentially 
expressed pathways were provided in Supplmentary Table S4. Interestingly, the top downregulated pathway under hypoxia was the TCA cycle with glycolysis and OXPHOS also in the top five.

One of Ref-1's major functions is its redox activity in which Ref-1 converts an oxidized transcription factor into a reduced transcription factor which leads to an increase in its DNA binding and functional activity. We quantitated the activity of various transcription factors after Ref-1 knockdown by identifying the gene co-regulation modules that are associated with different experimental conditions, i.e. hypoxia. ${ }^{44,64}$ Specifically, gene-wise expression states were first inferred by the left truncated mixture Gaussian model. Modules of genes that show consistent activated or suppressed expression in a subset of cells were identified using a non-negative matrix factorization method namely MEBF. ${ }^{45}$ We further evaluated the enrichment of the genes in each module against known targets of transcriptional regulatory factors, and the association of the cells of each module with the experimental conditions. Supplementary Table S5 lists the predicted transcriptional regulatory factors of each identified module (see details in Methods). Specifically, downregulation of the gene modules possibly regulated by CTCF, SP1, POLR2A, MAX, CEBPB, REST, MYC, JUN, JUND, NFKB1, STAT3, STAT1, HIF1A, FOXA1, and CREB1 were identified in siRef-1 vs Scr control under both hypoxia and normoxia condition, confirming interactions between Ref- 1 and its known transcriptional regulators.

\section{Metabolic shifts following treatment with Ref-1 inhibitor observed from scRNA-seq data}

Noting the scRNA-seq and proteomics data consistently revealed downregulated central metabolism pathways in siRef-1 vs Scr control under both hypoxia and normoxia, we specifically focused on characterizing the gene expression alterations in central metabolic pathways. These pathways included glycolysis, pentose phosphate pathway, lactate production, TCA cycle, oxidative phosphorylation, glutaminolysis, and other amino acid metabolism that can fuel the production of key metabolites succinate, fumarate, malate and oxaloacetate in the TCA cycle.

scRNA seq data presented in Fig. 2 illustrates the dysregulated enzymes involved in the selected central metabolic pathways in siRef-1 vs Scr control under hypoxia (Fig. 2A) and normoxia (Fig. 2B). Glycolysis $(p=5.7 e-6)$, TCA cycle $(p=1.1 e-7)$ and oxidative phosphorylation $(p=1.7 e-8)$ pathways were significantly enriched in downregulated genes in siRef-1 vs Scr control under hypoxia. In addition, the enzymes phosphogluconate dehydrogenase (PGD) and transaldolase (TALD01) that catalyze the first steps of glucose flow into pentose phosphate were significantly downregulated in siRef-1 vs Scr control. Significant downregulation of lactate dehydrogenase A (LDHA) was also observed. The enzymes asparagine synthetase (ASNS), argininosuccinate lyase (ASL), and adenylosuccinate lyase (ADSL) involved in the metabolism of aspartate to fumarate and oxaloacetate were also significantly downregulated. Under normoxia, the glycolysis $(p=7.5 e-7)$ and oxidative phosphorylation $(p=6.2 e-4)$ pathways were also significantly enriched in downregulated genes in siRef- 1 vs Scr control but were not as dramatically reduced compared to hypoxia (Fig. 2A, B). Although the TCA cycle, lactate production, and aspartate metabolic genes are downregulated under normoxia, the effects are more robust under 
hypoxia, and no significant changes in genes involved in glutaminolysis were observed when Ref-1 levels are reduced under either hypoxia or normoxia.

With the reduction in expression of genes within glycolysis, TCA cycle, and oxidative phosphorylation pathways, genes involved in the mitochondrial respiratory complexes I-IV as well as ATP synthases were also investigated. Figure $2 \mathrm{C}$ and $2 \mathrm{D}$ show the dramatic downregulation of genes in the mitochondrial respiratory complexes I-IV and ATP synthases. When Ref-1 is knocked down and the cells are exposed to hypoxia, significant downregulation of the mitochondrial complex I $(p=0.001)$, complex III $(p=0.03)$ and ATP synthase $(p=0.0004)$ genes were observed. Similarly, statistically significant but less downregulated mitochondrial complex I $(p=0.009)$ genes were observed under normoxia. Figure 2E highlights the expression profile of selected genes involved in these central metabolic pathways under the conditions of hypoxia/normoxia and Ref-1 knockdown.

Ref-1 inhibition directly effects mitochondrial OXPHOS complex genes.

Following the scRNA-seq and proteomic bioinformatic analysis in Figs. 1 and 2, gene expression changes were validated in $\mathrm{PaO3C}$ cells using a panel of six genes that are involved in OXPHOS within the various complexes of the electron transport chain (ETC). Knockdown of Ref-1 significantly downregulated the expression of this panel of six genes, confirming the scRNA-seq data (Fig. 3A). These six genes were not strongly induced by hypoxia ( 1.3-fold), but the downregulation was consistent when Ref-1 levels were reduced under both conditions. In order to delineate which function of Ref-1 was driving the decrease in gene expression of the mitochondrial complex genes seen in Fig. 3A, Pa03C cells were treated with APX2009 $(10 \mu \mathrm{M})$, a second-generation Ref-1 redox specific inhibitor, which has no effect on Ref-1 DNA repair endonuclease activity. ${ }^{65}$ All six genes were reduced following Ref- 1 redox inhibition in $\mathrm{Pa} 03 \mathrm{C}$ cells grown in monolayer, however NDUFS4 was not as dramatic as the results with Ref-1 knockdown (Fig. 3B). As 3D spheroid cultures of PDAC cells mimic in vivo tumor hypoxic regions, further evaluation of these genes was done using Pa03C spheroids. Similar downregulation was observed for mitochondrial complex genes as shown in Fig. 3C. In stark contrast, cancer-associated fibroblast (CAF) cells that would be found within the tumor microenvironment did not demonstrate the same changes in gene expression of these mitochondrial genes as was observed in the tumor cells under either hypoxia or normoxia (Fig. 3D).

Using an established panel of twelve genes that were significantly changed with Ref-1 siRNA treatment under normoxia, we evaluated the expression of these genes as markers of Ref- 1 inhibition under conditions of hypoxia and treatment with Ref-1 redox signaling inhibitor, APX2009. ${ }^{25}$ Under conditions of hypoxia, expression of CIRBP, ITGA1, NOTCH3, TAPBP, and CA9 was upregulated and then significantly reduced following Ref-1 redox inhibition with APX2009 with the exception of ITGA1 (Fig. 3E). Expression of ABCG2, COMMD7, ISYNA1, RAB3D, SIPA1, TAPBP, TNFAIP2, and BIRC5 was significantly reduced following Ref-1 redox inhibition with APX2009 regardless of the oxygen conditions. PPIF was downregulated under hypoxia and was upregulated with Ref-1 inhibition under normoxia as well as hypoxia. Two genes, ABCG2 and ITGA1 went opposite to that observed with Ref-1 knockdown ${ }^{25}$ (ABCG2 
was downregulated and ITGA1 was upregulated with APX2009 treatment, Fig. 3E). BIRC5 (Survivin) and CA9 were used as controls and were previously published as markers of Ref-1 redox inhibition (Fig. 3E). ${ }^{21,41}$

Again using 3D spheroid cultures of PDAC cells, further evaluation of these Ref-1 regulated genes was done. Similar yet more pronounced downregulation was observed for Ref-1-regulated gene subsets, with the exception of NOTCH3 (Fig. 3F). As seen previously, PPIF gene expression was significantly upregulated with Ref-1 redox inhibition using APX2009. Representative pictures of the 3D spheroids following treatment with vehicle (DMSO) or APX2009 are shown in Fig. 3G.

Functional validation of the defect in metabolic pathways following Ref-1 knockdown shows reduced TCA cycle substrates.

The effect of glucose levels in the growth conditions of both Scr control and siRef-1 was tested using 3D spheroid assays incorporating both patient-derived tumor cells as well as CAFs (Supplemental Fig. S1). $\mathrm{Pa03C}$ cells were transfected with Ref- 1 or Scr siRNA and cultured with or without CAFs in regular or lowglucose (LG) media. In presence and absence of CAFs, the Scr control spheroids grew significantly slower in the LG media compared to regular growth media. The cultures containing CAFs were less affected by the low glucose media, suggesting that the CAFs are providing that necessary tumor growth support (Supplemental Fig. S1B). However, when Ref-1 was knocked down, there was no difference in growth regardless of the media, further demonstrating that the main effect on growth of the spheroids was the lack of Ref-1 expression.

To confirm the gene expression and proteomic effects on TCA cycle and also investigate effects on fatty acid oxidation with functional data, a plate-based mitochondrial function assay that measures the electron flow through the electron transport chain was conducted (Supplemental Fig. S2A). This colorimetric assay measured the utilization rate of different metabolic substrates following knockdown of Ref-1 compared to Scr control. Multiple substrates involved in the TCA cycle exhibited significantly reduced rates of reaction after transfection with $10 \mathrm{nM}$ siRef-1 (efficiency of Ref-1 knockdown (99\%) shown in Fig. 4C) in comparison to $10 \mathrm{nM}$ Scr siRNA (Fig. 4A \& B, Supplemental Fig. S2C). Succinic Acid was reduced by $24 \%$, Fumaric Acid reduced by $22 \%$, and Malic Acid was down by $33 \%$. Changes in fatty acid oxidation was not observed under these conditons with these mitochondrial plates (not shown). The kinetic curves at $37^{\circ} \mathrm{C}$ for the four TCA cycle substrates within a representative experiment are shown in Figure S2.

Functional validation of the defect in metabolic pathways following pharmacological inhibition of Ref-1 redox activity and metabolic inhibitor, Devimistat.

In order to determine whether Ref-1 redox activity was responsible for the decrease in TCA substrate activity, mitochondrial function assays were performed following APX2009 treatment at 5 and $10 \mu \mathrm{M}$. The four TCA cycle substrates (a-Keto-Glutaric Acid, Succinic Acid, Fumaric Acid, and Malic Acid, Supplemental Fig. S2A, C) displayed significantly reduced rates of reaction $(30-72 \%)$ in response to $5 \mu \mathrm{M}$ 
APX2009 treatment, and further dose-dependent reduction was observed when treated with $10 \mu \mathrm{M}$ APX2009. These results confirm that Ref- 1 redox activity plays a role in tumor cells' ability to utilize TCA cycle substrates (Fig. 4D, E and S2C). As a negative control for this redox inhibition, an inactive analog of APX2009, RN7-58, was evaluated for its ability to block mitochondrial function, and no inhibition of TCA substrates was observed (Fig. 4F, G and S2D). ${ }^{66}$ We also used a metabolic inhibitor, Devimistat as a positive control to compare and validate the effects on the TCA cycle by APX2009. Devimistat was able to significantly reduce two of the TCA cycle substrates (Malic acid by $20-30 \%$ and Fumaric Acid by $35 \%$ at $50 \mu \mathrm{M}$, Fig. $4 \mathrm{H}$ and S2F). Similar to changes in mitochondrial complex gene expression in CAFs, APX2009 did not affect mitochondrial function within CAF02 cells (Fig. 4I and S2G). Line graphs showing kinetic curves at $37^{\circ} \mathrm{C}$ for the four TCA cycle substrates are represented by Supplemental Figures S2D-G.

Ref-1 redox inhibitor APX2009 and metabolic inhibitor Devimistat exhibit similar tumor reduction in in vivo xenograft studies

To determine whether the inhibition of Ref-1 leading to a reduction in mitochondrial gene expression and TCA substrate utilization would translate to a blockade of tumor growth in vivo, Pa03C cells were implanted in mice and treated with either APX2009 $(35 \mathrm{mg} / \mathrm{Kg})$ or metabolic inhibitor, Devimistat $(50 \mathrm{mg} / \mathrm{Kg})$. Both treatments resulted in a significant decrease in tumor volume over time starting at Day $17(p<0.0001$, Fig. $5 A)$. Growth rate per day of tumor volume among the groups was determined to be significantly different using the repeated measure regression model $(p<0.0001)$. Tumor weights also showed a significant decrease compared to the vehicle control group $(p<0.05)$ (Fig. 5B), with APX2009 and Devimistat showing a 57\% reduction in tumor weight. Both treatments were well tolerated as there was no significant change in body weights (Fig. 5C).

Due to the importance of the CAFs in the microenvironment and their impact on response to therapy, a tumor model with co-implantation of low passage pancreatic cancer cells, Panc10.05 and CAF19 cells was utilized. Similar to the results in Fig. 5A, treatment with either APX2009 (35 mg/ $\mathrm{Kg}$ ) or Devimistat $(50 \mathrm{mg} / \mathrm{Kg})$ resulted in a significant decrease in tumor volume $(p<0.0001$, Fig. 5D). At the time of harvest, while both treatments showed a decrease in tumor weight, only APX2009 resulted in a statistically significant decrease by $\sim 61 \%$ ( $p<0.05$, Fig. $5 \mathrm{E}$ ). No significant change in body weights was observed (Fig. 5F). Tumors were collected and stained for H\&E and Vimentin. Vimentin staining shows the presence of CAFs even after tumor shrinkage with APX2009 treatment (Fig. 5G and Supplemental Fig. S3).

Inhibition of Ref-1 redox activity in combination with metabolic inhibitor Devimistat significantly reduces tumor growth in two 3D models with simulated PDAC microenvironment

To assess whether the combination of APX2009 and Devimistat would synergize to prevent further tumor growth, two 3D co-culture assays were utilized. These included a 3D co-culture spheroid assay and an interstitial tumor-microenvironment-on-a-chip (iT-MOC) assay. Our group previously published doses of APX compounds that block spheroid growth in the 3D co-culture model. ${ }^{53}$ Hence, the dose response of 
Devimistat as a single agent is shown in Pa03C (Fig. 6B, left panel) and in Panc10.05 cells (Fig. 6E, left panel). APX2009, parent drug APX3330 as well as additional analog APX2014, were used to inhibit Ref-1 redox activity as single agent and in combination with Devimistat (Fig. 6B, C, E, F and Supplemental Figure S4A). Single agent Devimistat or APX2009 resulted in significant reduction in spheroid growth as visualized through fluorescence intensity (Fig. 6A-F). However, combination treatment of Ref-1 inhibitor plus Devimistat resulted in further reduction of spheroid growth of the tumor cells (Fig. 6B \& 6E). APX2009 + Devimistat had combination index $(\mathrm{Cl})$ values ranging from 0.37-0.74 indicating moderate to strong synergy (see Supplemental Table 6 for all values). Importantly, the single agent and combination treatments had little to no effect on CAF19 growth (Fig. 6A-F, with the exception of combination treatment in the 10.05 + CAF19 co-cultures (Fig. 6F, right panel, S4A). Supplemental Figure S4A shows similar results with the Ref-1 redox inhibitors, APX3330 (completed Phase I clinical trial) and APX2014 in combination with Devimistat.

Combination drug experiments were also performed using the iT-MOC assays with the co-culture of Panc10.05 and CAF19 cells. ${ }^{67}$ This assay was utilized due to its ability to reconstitute the interstitial transport in a 3D matrix (Fig. 6G). The capillary channel mimics blood-borne drug transport along a capillary vessel. The porous membrane simulates transvascular transport. The interstitial channel where pancreatic cancer cells and CAFs are co-cultured in the 3D matrix aims to reconstitute the interstitial transport. Two side drainage channels correspond to lymphatic drainage. The drug transport is achieved by applying a hydrostatic pressure difference between the capillary channel and lymphatic channel, simulating elevated interstitial fluid pressure (IFP) range in PDAC. Transport properties of iT-MOC have been measured and compared with in vivo tumors previously. ${ }^{57,58}$

Figure $6 \mathrm{H}$ represents the treatment timeline for iT-MOC assay. Representative fluorescence micrographs of iT-MOC samples during the experiment are shown in Fig. 6l. In the control group, both cell types proliferated significantly over time as indicated by red and green fluorescence (Fig. 6I, S4B). In terms of cell growth (Fig. S4B) and survival (Fig. 6J), no notable difference is observed in cancer cell growth among the experimental groups until Day 5. After the second treatment at Day 5, tumor cells treated with a combination of APX2009 and Devimistat grew much slower at $48 \%$ of the control group compared to either single agent $(p<0.05$, Fig. $6 \mathrm{~J} \& S 4 \mathrm{~B})$. In all experimental groups, no notable difference in the proliferation of CAFs is noted (Fig. 6J \& S4C) indicating that the combination treatment was more dramatically affecting the tumor even in the presence of the CAFs and was similar in both 3D co-culture assays.

Inhibition of Ref-1 redox activity in combination with metabolic inhibitor Devimistat reduces mitochondrial gene expression and causes TCA cycle disruption

Based on the 3D data demonstrating efficacy of the combination treatment, we further investigated the effects on gene expression of combination treatment in $\mathrm{Pa} 03 \mathrm{C}$ spheroids. Of the six previously tested OXPHOS mitochondrial genes found from the scRNA-seq data, the combination treatment (blue bars) significantly reduced the levels of UQCRC1, SURF1, COX15 and NNT compared to either of the single 
agents used (Fig. 7A). Within the Ref-1-regulated 12 gene panel, combination treatment reduced the levels of NOTCH3 (which was not affected by either single agent) and RAB3D while increasing the levels of PPIF significantly compared to either singles. The effects of combination treatment on ABCG2, CIRBP, COMMD7, ISYNA1, PRDX5 and TNFAIP2 were significantly down compared to either APX2009 alone or Devimistat alone, however the combination effects were most dramatic in NOTCH3 and RAB3D. BIRC5 and CA9 were used as controls (Fig. 7B).

Following this, we analyzed whether the reduced gene expression with combination treatment had a further functional effect on the TCA cycle. For this, a combination of Devimistat $(50 \mu \mathrm{M})$ with APX2009 $(5$ or $10 \mu \mathrm{M}$ ) was used. Devimistat with APX2009 at $10 \mu \mathrm{M}$ had a greater effect on reducing the rate of reaction of all four TCA substrates compared to APX2009 or Devimistat alone (a-Keto-Glutaric Acid $42 \%$ or $58 \%$, Succinic Acid $-30 \%$ or $64 \%$, Fumaric Acid $-25 \%$ or $12.5 \%$, and Malic Acid $-32 \%$ or $46 \%$ ) (Fig. 7C). Representative picture of the reduction in colorimetric assay shown in Fig. 7D. The effects were also dose-dependent. As the amount of APX2009 increased, the TCA substrate utilization was decreased. Line graphs showing kinetic curves (Supplemental Figures S5) at $37^{\circ} \mathrm{C}$ for the four TCA cycle substrates are represented.

\section{Discussion}

A hallmark of PDAC is its capability to reprogram metabolism. Pancreatic cancer's ability to adapt to nutrient and oxygen fluctuation results in severe therapeutic resistance. Communicating with its tumor microenvironment (TME) through reciprocal upregulation or downregulation of several metabolic pathways like aerobic glycolysis, oxidative phosphorylation, glutaminolysis, lipogenesis and lipolysis, autophagic status, and anti-oxidative stress enables PDAC to thrive under nutrient deficiency and low oxygen conditions, as well as evade therapeutic death. KRAS oncogenic driver mutations are responsible for these tumor cells highly relying on OXPHOS for survival (by supplying ATP) as well as drug resistance (via multidrug transporters) ${ }^{68,69}$ Current options for the treatment of PDAC patients are FOLFIRINOX and gemcitabine + nab-paclitaxel. However these multi-agent options are still not adequate to cure pancreatic cancer and are often toxic to the patients. ${ }^{70}$ Hence, this study investigates the targeting of metabolic pathways through Ref- 1 inhibition as an option for therapy.

Our scRNA-seq analysis of $\mathrm{Pa} 03 \mathrm{C}$ cells transfected with Scr or siRef- 1 under hypoxia identified novel pathways and transcriptional modules regulated by Ref-1, thereby opening up new avenues to treat PDAC. As represented in the Fig. 1D panel 2, pathway enrichment analysis revealed TCA cycle, lipid metabolism, glycolysis, OXPHOS and HIF1a pathways as the top five to be downregulated with Ref-1 knockdown under hypoxia. Further detailed analysis combining scRNA-seq, proteomics, and functional assays revealed the enzymes involved in those selected pathways to be clustered to TCA cycle and OXPHOS (Fig. 2). With the intent of validating these effects, we selected a set of genes representive of the above pathways (Fig. 3A) and observed a significant reduction in their levels with Ref- 1 knockdown as well as Ref-1 redox signaling inhibition with APX2009. Similar to previous studies surveying pathways affected by Ref-1 silencing in cancer cells, mitochondrial function was found as one of the most 
significantly changed, including our previous scRNA seq study in PDAC cells. ${ }^{25,71,72}$ What was not clear from these studies was whether the DNA BER function or the redox signaling function of Ref- 1 was responsible for this perturbation of mitochondrial function. The results presented here demonstrate that a second generation, more potent Ref-1 redox inhibitor, APX2009, significantly downregulated all OXPHOSassociated genes (Fig. 3B). This points toward the transcriptional regulation of OXPHOS through Ref-1 redox signaling as the main determinant of the effects on mitochondrial function when Ref- 1 is knocked down.

Another interesting gene expression change is the upregulation of PPIF (peptidylprolyl isomerase $\mathrm{F}$ or cyclophilin D). PPIF is part of the mitochondrial permeability transition pore (PTP) that is invoved in the induction of cell death. ${ }^{73}$ Interestingly, under hypoxia PPIF is significantly downregulated, presumably one of the many transcriptional changes that allow cancer cells to survive hypoxic stress. However, with APX2009 or Devimistat treatment or combination of the two, the levels of PPIF are increased about 5-10fold (Fig. 7B) indicating that inhibition of TCA is likely increasing tumor cell death. The inhibition of TCA cycle activity was confirmed with APX2009, Devimistat, or combination therapy using a functional assay. Either agent used alone affected the TCA substrates with APX2009 being more potent than Devimistat. These data indicate that Ref- 1 redox signaling inhibition reduces the activity of the TCA cycle similar to Devimistat, but at a 10 fold lower concentration, leading to cell death.

CAFs, which are most abundant in the TME, form a critical component of the PDAC milieu. CAFs produce lactate from glycolysis that fuels OXPHOS in the PDAC cells for ATP production via alpha-ketoglutarate, isocitrate dehydrogenase and pyruvate dehydrogenase enzymes. The production of L-lactate, ketones, free fatty acids, and glutamine by CAFs may also drive tumor cell growth through metabolic coupling and enabling the tumor to utilize OXPHOS. HIF1a, NFKB, and loss of Cav-1 have been implicated in the signaling that drives autophagy and catabolism in CAFs that can directly affect tumor growth. Thus, tumors switch between OXPHOS and glycolysis under diverse microenvironmental conditions. ${ }^{74}$ Both transcription factors, HIF1a and NFKB are targets of Ref- 1 redox signaling activity. ${ }^{75-77}$ Hence, targeting both OXPHOS in cancer cells as well as glycolysis in CAFs may prove effective, with the caveat that toxicity to non-cancerous cells must be considered. ${ }^{76,78}$ Ref-1 redox inhibition showed minimal effects on the expression of mitochondria complex genes and on mitochondria function in the CAFs (Fig. 3D, 4I). Noticeably, the effects of Ref-1 inhibition on mitochondrial function is not the same between tumor and CAFs, pointing again toward a different mechanism of metabolic reprogramming regarding tumor and CAF cells (Fig. 4I). Furthermore, the effects of Devimistat as a single agent or in combination therapy in the 3D models shown in Fig. 6 demonstrate that targeting of the TCA cycle is more cytotoxic to the tumor cells than the CAFs. The 3D co-culture spheroids consisting of both tumor cells and CAFs demonstrate regions of hypoxia and growth factor gradients. ${ }^{53} 3 \mathrm{D}$ coculture assays demonstrated that the combination treatment was not only more effective than the single agents, but that the treatments in general targeted the tumor cells more dramatically than the surrounding microenvironment with minimal effects on the CAFs (Fig. 6A-F). In the iT-MOC system, fluorescence from both cell types significantly increased over time, while in the 3D spheroid based assay the CAFs are less proliferative than the tumors 
(Fig. 6 and Supplementary Fig. S4). Likewise, combination of APX2009 with Devimistat proved to significantly reduce the expression of all six mitochondrial complex genes when compared to single agents in 3D spheroids consisting of Pa03C cells (Fig. 7A). Devimistat is inhibiting PDH (pyruvate dehydrogenase) and aKGDH (a-ketoglutarate dehydrogenase) and thereby blocking TCA cycle activity, whereby inhibition of Ref- 1 appears to dramatically affect the expression of several genes involved in various aspects of metabolism including glycolysis, TCA cycle, and ETC, with TCA cycle being the most downregulated pathway following knockdown in hypoxia (Fig. 1D). Future studies will further define the metabolic pathways that are utilized during varying oxygen levels in the presence and absence of Ref-1 and whether there is a shift to TCA cycle at extreme oxygen deprivation as described in ${ }^{26}$. The ROS levels generated during hypoxia as well as Ref- 1 knockdown are of interest, as well as activaton of NFKB. ${ }^{28}$ Ostensibly, the combination therapy is further shutting down the tumor cells' ability to generate energy and leading to a decrease in 3D spheroid tumor growth. Tumor-selective killing is desirable in PDAC therapeutic approaches, as attempts to modulate the PDAC stroma to improve treatment response have not been well translated and, in fact, have shown to hinder therapeutic approaches. Due to an unanticipated anti-tumor role of select stroma components, the ablation of CAFs or targeting of certain ECM proteins in the stroma led to changes that actually accelerated tumor growth and impaired treatment outcome. ${ }^{79-81}$

To demonstrate that inhibition of Ref-1 redox activity blocks tumor growth in vivo and also assess the effects on the CAFs, low passage PDAC cells were implanted alongside CAFs into NSG mice. Treatment with either APX2009 or Devimistat which should lead to a decrease in cancer cell metabolism did indeed translate to a significant decrease in tumor volume (Fig. 5A \& 5D) as well as tumor weight (Fig. 5B \& 5E) and did not obliterate the CAFs as indicated by vimentin positivity. These in vivo xenograft results suggest that targeting the metabolic capacity of PDAC tumors will slow their growth rate without killing the associated stroma have strong translational implications.

Several of the transcription factors that interact with Ref- 1 have a profound impact on metabolic reprogramming in cancer, including HIF1-a, STAT3, and NRF2 (nuclear factor erythroid 2-related factor 2). ${ }^{82,83} \mathrm{NRF} 2$ is a transcription factor that can act in a protective mechanism in cancer cells from oxidative and chemical stresses by controlling their redox balance, regulation of antioxidant genes, and metabolic reprogramming. ${ }^{83}$ Interestingly, Ref-1 redox signaling activity activates HIF1-a and STAT3 while the opposite is true of NRF2. Conceivably by blocking Ref-1 redox activity, the tumor cells' ability to switch to aerobic glycolysis is attenuated and thereby the tumor cells are less proliferative. Our previously published work demonstrated that, selective inactivation of Ref-1 leads to upregulation of NRF2 target genes like HMOX-1. ${ }^{23}$ This could be the cellular response to the blockade by Ref- 1 on the TCA cycle. Taken together from our current work, it is clear that more research is needed to fully understand the intersection of Ref- 1 signaling and transcriptional regulation of metabolism and its impact on cancer cell growth.

\section{Conclusion}


To summarize, this is the first study to identify Ref-1's role in regulating glycolysis and mitochondrial metabolism through the integration of single cell RNA seq, proteomics, and mitochondrial function under hypoxia and Ref-1 deficient redox signaling conditions. Ref-1's redox signaling / regulatory role plays a crucial function in mitochondrial metabolism, specifically altering the TCA cycle and gene expression within OXPHOS / ETC thereby enabling the survival of PDAC even under nutrient and oxygen stress. We used 3D spheroids in monoculture as a mechanistic model or as co-culture with CAFs as a means to demonstrate growth regulation by Ref-1 both in vitro as well as in vivo. In these models, we determined that Ref-1 inhibition leads to significant reduction in PDAC growth and in regulation of gene expression that leads to the dysregulation of many genes within Complex 1 of the ETC especially under hypoxia (Fig. 2C).

As mitochondria can also generate reactive oxygen species (ROS) that damage nuclear and mitochondrial DNA, the role of Ref-1's BER function was hypothesized to also be important in the observed phenotype, as seen in the experiments with Ref-1 siRNA knockdown. Several studies including ours demonstrated that the repair role of Ref-1 (APE1) is essential to maintain the integrity of mitochondria after oxidative stress. ${ }^{24,84,85}$ Ref- 1 's redox regulation of transcription factors leading to gene expression changes that ultimately regulate mitochondrial function has been less explored. APX3330 and its analogs, APX2009 and others, specifically target Ref-1's redox signaling activity, but not the DNA repair function. ${ }^{65}$ Therefore, this study in addition to exploring the consequences of targeting mitochondrial dysfunction in PDAC, demonstrates that the redox activity of Ref-1 plays a novel role in metabolic signaling through disruption of the TCA cycle and the ETC. Although many studies have shown mitochondrial perturbation following Ref- 1 blockade, we are the first to implicate the redox signaling function in this phenotype.

\section{Abbreviations}

AP-1

Activator protein-1

BER

Base excision repair

CAFs

cancer-associated fibroblasts

DEGs

differentially expressed genes

DMSO

dimethylsulfoxide

ECM

extracellular matrix

ETC

electron transport chain 
HIF1-a

hypoxia inducible factor 1 - alpha

HMOX-1

heme oxygenase 1

IFP

interstitial fluid pressure

iT-MOC

Interstitial tumor-microenvironment-on-chip

NFkB

nuclear factor kappa b

NSG

NOD scid gamma (NOD.Cg-Prkdc $c^{s c i d} / / 2 \operatorname{rg}^{t m 1} 1 \mathrm{Wj} / \mathrm{SzJ}$ )

NRF2

Nuclear factor erythroid 2-related factor 2

OXPHOS

oxidative phosphorylation

PDAC

pancreatic ductal adencarcinoma

$\mathrm{PDH}$

pyruvate dehydrogenase

PKT

Propylene Glycol, Kolliphor HS15, Tween 80

qRT-PCR

quantitative real time-polymerase chain reaction

Ref-1/APE1

Redox factor 1/Apurinic-apyrimidinic endonuclease 1

ROS

reactive oxygen species

$\mathrm{Scr}$

Scrambled

scRNA-seq

single cell RNA sequencing

STAT3

signal transducer and activator of transcription 3

TCA cycle

tricarboxylic acid cycle

TF

transcription factors

TME

tumor microenvironment 


\section{Declarations}

Acknowledgements:

Work in the IUSM Proteomics Core was supported, in part, with support from the Indiana Clinical and Translational Sciences Institute which is funded by Award Number UL1TR002529 from the National Institutes of Health, National Center for Advancing Translational Sciences, Clinical and Translational Sciences Award. Acquisition of the IUSM Proteomics core instrumentation used for this project, the Orbitrap Fusion Lumos, was provided by the Indiana University Precision Health Initiative. IUSCCC also supports the proteomics core on the CCSG grant.

\section{Authors' contributions:}

Designed the study - F.S., S.G., M.R.K., H.M., B.H., C.Z., and M.L.F.; Performed experiments, validation and analysis - S.G., F.S., X.L., H.M., A.L.M., B.H., C.Z. and M.L.F; Imaging (investigation and data analysis) G.S. and E.H.; Help with drug combination analysis - L.A.; Writing (Original Draft) - S.G., F.S., C.Z. and M.L.F.; Writing (Review \& Editing) - S.G., F.S., H.M., B.H., C.Z., M.R.K. and M.L.F.; Consultation - M.I.; Supervision and Funding Acquisition - B.H., M.R.K. and M.L.F.

\section{Funding:}

M.L.F., M.R.K., A.L.M., C.Z. supported by IUSCC Cancer Center Support grant (P30 CA082709). B.H. and H.M. supported by a Challenge Award from the Purdue Center for Cancer Research (P30 CA023168). M.R.K. and M.L.F. were supported by grants from the National Institute of Health and National Cancer Institute R01CA167291 and R01CA167291-S1. Dr. Kelley was also supported by NIH/NCl grants R01CA205166, R01CA231267 and R01HL140961. Dr. Fishel and Dr. Han were supported by NIH/NCl grant U01HL143403 and CTSI grant UL1 TR002529. Dr. Fishel was also supported by R01CA211098 and R01NF180045. C.Z. was supported through NIGMS (1R01GM131399). M.L.F. and M.R.K. were additionally supported by the Riley Children's Foundation.

\section{Availability of data and materials:}

The previously published scRNA-seq data under normoxia can be found in the GEO, accession number GSE99305. The mass spectrometry proteomics data have been deposited to the ProteomeXchange Consortium via the PRIDE ${ }^{86}$ partner repository with the dataset identifier PXD020515 and $10.6019 /$ PXD 020515. The single cell hypoxia data is currently being submitted to the GEO database.

\section{Ethics approval and consent to participate:}

All animal studies were conducted under the guidelines of the National Institutes of Health and were approved by the Institutional Animal Care and Use Committee of Indiana University School of Medicine.

\section{Consent for publication:}


Not applicable.

\section{Competing interests:}

Mark R. Kelley has licensed APX3330 through Indiana University Research and Technology Corporation to Apexian Pharmaceuticals LLC. APX2009 and APX2014 are second generation compounds from Apexian Pharmaceuticals. Apexian Pharmaceuticals had neither control nor oversight of the studies, interpretation, or presentation of the data in this manuscript.

\section{References}

1 Siegel, R. L., Miller, K. D. \& Jemal, A. Cancer statistics, 2019. CA Cancer J Clin69, 7-34, doi:10.3322/caac.21551 (2019).

2 Bijlsma, M. F. \& van Laarhoven, H. W. The conflicting roles of tumor stroma in pancreatic cancer and their contribution to the failure of clinical trials: a systematic review and critical appraisal. Cancer Metastasis Rev, doi:10.1007/s10555-014-9541-1 (2015).

3 Longati, P. et al. 3D pancreatic carcinoma spheroids induce a matrix-rich, chemoresistant phenotype offering a better model for drug testing. BMC Cancer13, 95, doi:10.1186/1471-2407-13-95 (2013).

4 Chang, Q., Jurisica, I., Do, T. \& Hedley, D. W. Hypoxia predicts aggressive growth and spontaneous metastasis formation from orthotopically grown primary xenografts of human pancreatic cancer. Cancer Res71, 3110-3120, doi:10.1158/0008-5472.CAN-10-4049 (2011).

5 Rucki, A. A. et al. Heterogeneous Stromal Signaling within the Tumor Microenvironment Controls the Metastasis of Pancreatic Cancer. Cancer Res, doi:10.1158/0008-5472.CAN-16-1383 (2016).

6 Chaika, N. V. et al. Differential expression of metabolic genes in tumor and stromal components of primary and metastatic loci in pancreatic adenocarcinoma. PLoS One7, e32996, doi:10.1371/journal.pone.0032996 (2012).

7 Ngoi, N. Y. L. et al. Targeting Cell Metabolism as Cancer Therapy. Antioxid Redox Signa/32, 285-308, doi:10.1089/ars.2019.7947 (2020).

8 Biancur, D. E. \& Kimmelman, A. C. The plasticity of pancreatic cancer metabolism in tumor progression and therapeutic resistance. Biochim Biophys Acta Rev Cancer1870, 67-75, doi:10.1016/j.bbcan.2018.04.011 (2018).

9 Zhang, J., Pavlova, N. N. \& Thompson, C. B. Cancer cell metabolism: the essential role of the nonessential amino acid, glutamine. EMBO J.36, 1302-1315, doi:10.15252/embj.201696151 (2017).

10 DeBerardinis, R. J. \& Cheng, T. Q's next: the diverse functions of glutamine in metabolism, cell biology and cancer. Oncogene29, 313-324, doi:10.1038/onc.2009.358 (2010). 
11 Smolkova, K. et al. Waves of gene regulation suppress and then restore oxidative phosphorylation in cancer cells. Int J Biochem Cell Bio/43, 950-968, doi:10.1016/j.biocel.2010.05.003 (2011).

12 Fan, J. et al. Glutamine-driven oxidative phosphorylation is a major ATP source in transformed mammalian cells in both normoxia and hypoxia. Mol. Syst. Biol.9, 712, doi:10.1038/msb.2013.65 (2013).

13 Seagroves, T. N. et al. Transcription factor HIF-1 is a necessary mediator of the pasteur effect in mammalian cells. Mol. Cell. Biol.21, 3436-3444, doi:10.1128/MCB.21.10.3436-3444.2001 (2001).

14 Robin, E. D., Murphy, B. J. \& Theodore, J. Coordinate regulation of glycolysis by hypoxia in mammalian cells. J Cell Physio/118, 287-290, doi:10.1002/jcp.1041180311 (1984).

15 Fung, H. \& Demple, B. A vital role for Ape1/Ref1 protein in repairing spontaneous DNA damage in human cells. Mol Cel/17, 463-470 (2005).

16 Izumi, T. et al. Two essential but distinct functions of the mammalian abasic endonuclease. Proc Natl Acad Sci U S A102, 5739-5743, doi:10.1073/pnas.0500986102 (2005).

17 Jiang, Y. et al. Role of APE1 in differentiated neuroblastoma SH-SY5Y cells in response to oxidative stress: use of APE1 small molecule inhibitors to delineate APE1 functions. DNA Repair (Amst)8, 12731282, doi:S1568-7864(09)00214-6 [pii] 10.1016/j.dnarep.2009.08.003 (2009).

18 Kelley, M. R., Logsdon, D. \& Fishel, M. L. Targeting DNA repair pathways for cancer treatment: what's new? Future Onco/10, 1215-1237, doi:10.2217/fon.14.60 (2014).

19 Gaiddon, C., Moorthy, N. C. \& Prives, C. Ref-1 regulates the transactivation and pro-apoptotic functions of p53 in vivo. EMBO J18, 5609-5621, doi:10.1093/emboj/18.20.5609 (1999).

20 Lando, D., Pongratz, I., Poellinger, L. \& Whitelaw, M. L. A redox mechanism controls differential DNA binding activities of hypoxia-inducible factor (HIF) 1alpha and the HIF-like factor. J Biol Chem275, 46184627 (2000).

21 Cardoso, A. A. et al. APE1/Ref-1 Regulates STAT3 Transcriptional Activity and APE1/Ref-1-STAT3 Dual-Targeting Effectively Inhibits Pancreatic Cancer Cell Survival. PLoS ONE7, e47462, doi:10.1371/journal.pone.0047462 (2012).

22 Kelley, M. R., Georgiadis, M. M. \& Fishel, M. L. APE1/Ref-1Role in Redox Signaling: Translational Applications of Targeting the Redox Function of the DNA Repair/Redox Protein APE1/Ref-1. Current molecular pharmacology5, 36-53 (2012).

23 Fishel, M. L. et al. Apurinic/apyrimidinic endonuclease/redox factor-1 (APE1/Ref-1) redox function negatively regulates NRF2. J Biol Chem290, 3057-3068, doi:10.1074/jbc.M114.621995 (2015). 
24 Shah, F. et al. Exploiting the Ref-1-APE1 node in cancer signaling and other diseases: from bench to clinic. npj Precision Oncology1, doi:10.1038/s41698-017-0023-0 (2017).

25 Shah, F. et al. APE1/Ref-1 knockdown in pancreatic ductal adenocarcinoma - characterizing gene expression changes and identifying novel pathways using single-cell RNA sequencing. Molecular oncology11, 1711-1732, doi:10.1002/1878-0261.12138 (2017).

26 Eales, K. L., Hollinshead, K. E. \& Tennant, D. A. Hypoxia and metabolic adaptation of cancer cells. Oncogenesis5, e190, doi:10.1038/oncsis.2015.50 (2016).

27 Vaziri-Gohar, A., Zarei, M., Brody, J. R. \& Winter, J. M. Metabolic Dependencies in Pancreatic Cancer. Front Onco/8, 617, doi:10.3389/fonc.2018.00617 (2018).

28 Tang, K. et al. Hypoxia-reprogrammed tricarboxylic acid cycle promotes the growth of human breast tumorigenic cells. Oncogene38, 6970-6984, doi:10.1038/s41388-019-0932-1 (2019).

29 Sharbeen, G., McCarroll, J., Goldstein, D. \& Phillips, P. Exploiting Base Excision Repair to Improve Therapeutic Approaches for Pancreatic Cancer. Frontiers in Nutrition2, doi:10.3389/fnut.2015.00010 (2015).

30 Caston, R. A. et al. The multifunctional APE1 DNA repair-redox signaling protein as a drug target in human disease. Drug discovery today, doi:10.1016/j.drudis.2020.10.015 (2020).

31 Fishel, M. L. et al. Impact of APE1/Ref-1 redox inhibition on pancreatic tumor growth. Mol Cancer Ther10, 1698-1708, doi:1535-7163.MCT-11-0107 [pii] 10.1158/1535-7163.MCT-11-0107 (2011).

32 Jiang, Y., Zhou, S., Sandusky, G. E., Kelley, M. R. \& Fishel, M. L. Reduced expression of DNA repair and redox signaling protein APE1/Ref-1 impairs human pancreatic cancer cell survival, proliferation, and cell cycle progression. Cancer Investigation28, 885-895 (2010).

33 Kelley, M. R. et al. Identification and Characterization of New Chemical Entities Targeting Apurinic/Apyrimidinic Endonuclease 1 for the Prevention of Chemotherapy-Induced Peripheral Neuropathy. J Pharmacol Exp Ther359, 300-309, doi:10.1124/jpet.116.235283 (2016).

34 Mcllwain, D. W., Fishel, M. L., Boos, A., Kelley, M. R. \& Jerde, T. J. APE1/Ref-1 redox-specific inhibition decreases survivin protein levels and induces cell cycle arrest in prostate cancer cells. Oncotarget 9 , 10962-10977 (2018).

35 Sardar Pasha, S. P. B. et al. Ref-1/APE1 Inhibition with Novel Small Molecules Blocks Ocular Neovascularization. J Pharmacol Exp Ther367, 108-118, doi:10.1124/jpet.118.248088 (2018).

36 Fishel, M. L. et al. Antitumor Activity and Mechanistic Characterization of APE1/Ref-1 Inhibitors in Bladder Cancer. Mol Cancer Ther18, 1947-1960, doi:10.1158/1535-7163.MCT-18-1166 (2019). 
37 Alistar, A. et al. Safety and tolerability of the first-in-class agent CPI-613 in combination with modified FOLFIRINOX in patients with metastatic pancreatic cancer: a single-centre, open-label, dose-escalation, phase 1 trial. Lancet Oncol, doi:10.1016/S1470-2045(17)30314-5 (2017).

38 Lee, K. C. et al. Translational assessment of mitochondrial dysfunction of pancreatic cancer from in vitro gene microarray and animal efficacy studies, to early clinical studies, via the novel tumor-specific anti-mitochondrial agent, CPI-613. Ann Trans/ Med2, 91, doi:10.3978/j.issn.2305-5839.2014.05.08 (2014).

39 Stuart, S. D. et al. A strategically designed small molecule attacks alpha-ketoglutarate dehydrogenase in tumor cells through a redox process. Cancer Metab2, 4, doi:10.1186/2049-3002-2-4 (2014).

40 Jones, S. et al. Core signaling pathways in human pancreatic cancers revealed by global genomic analyses. Science321, 1801-1806, doi:1164368 [pii]

10.1126/science.1164368 (2008).

41 Logsdon, D. P. et al. Regulation of HIF1alpha under Hypoxia by APE1/Ref-1 Impacts CA9 Expression: Dual Targeting in Patient-Derived 3D Pancreatic Cancer Models. Mol Cancer Ther15, 2722-2732, doi:10.1158/1535-7163.MCT-16-0253 (2016).

42 Richards, K. E. et al. Cancer-associated fibroblast exosomes regulate survival and proliferation of pancreatic cancer cells. Oncogene, doi:10.1038/onc.2016.353 (2016).

43 Principe, D. R. et al. Long-Term Gemcitabine Treatment Reshapes the Pancreatic Tumor Microenvironment and Sensitizes Murine Carcinoma to Combination Immunotherapy. Cancer Res, doi:10.1158/0008-5472.CAN-19-2959 (2020).

44 Wan, C. et al. LTMG: a novel statistical modeling of transcriptional expression states in single-cell RNA-Seq data. Nucleic Acids Res, doi:10.1093/nar/gkz655 (2019).

45 Wan, C. et al. in Association for the Advancement of Artificial Intelligence (2019).

46 Subramanian, A. et al. Gene set enrichment analysis: a knowledge-based approach for interpreting genome-wide expression profiles. Proc Natl Acad Sci U S A102, 15545-15550, doi:10.1073/pnas.0506580102 (2005).

47 Peck Justice, S. A. et al. Mutant thermal proteome profiling for characterization of missense protein variants and their associated phenotypes within the proteome. $\mathrm{J} \mathrm{Biol} \mathrm{Chem,}$ doi:10.1074/jbc.RA120.014576 (2020).

48 Levasseur, E. M. et al. Hypusine biosynthesis in beta cells links polyamine metabolism to facultative cellular proliferation to maintain glucose homeostasis. Sci Signa/12, doi:10.1126/scisignal.aax0715 (2019). 
49 Fan, Z. et al. Cleaving the oxidative repair protein Ape1 enhances cell death mediated by granzyme A. Nat Immunol4, 145-153 (2003).

50 Wang, D., Luo, M. \& Kelley, M. R. Human apurinic endonuclease 1 (APE1) expression and prognostic significance in osteosarcoma: enhanced sensitivity of osteosarcoma to DNA damaging agents using silencing RNA APE1 expression inhibition. Mol Cancer Ther3, 679-686 (2004).

51 Fishel, M. L. et al. Knockdown of the DNA repair and redox signaling protein Ape1/Ref-1 blocks ovarian cancer cell and tumor growth. DNA Repair (Amst)7, 177-186, doi:S1568-7864(07)00330-8 [pii] 10.1016/j.dnarep.2007.09.008 (2008).

52 Fishel, M. L., Colvin, E. S., Luo, M., Kelley, M. R. \& Robertson, K. A. Inhibition of the Redox Function of APE1/Ref-1 in Myeloid Leukemia Cell Lines Results in a Hypersensitive Response to Retinoic Acidinduced Differentiation and Apoptosis. Exp Hemato/38, 1178-1188, doi:S0301-472X(10)00402-9 [pii] 10.1016/j.exphem.2010.08.011 (2010).

53 Logsdon, D. P. et al. Blocking HIF signaling via novel inhibitors of CA9 and APE1/Ref-1 dramatically affects pancreatic cancer cell survival. Sci Rep8, 13759, doi:10.1038/s41598-018-32034-9 (2018).

54 Sempere, L. F., Gunn, J. R. \& Korc, M. A novel 3-dimensional culture system uncovers growth stimulatory actions by TGFbeta in pancreatic cancer cells. Cancer Biol Ther12, 198-207 (2011).

55 Arpin, C. C. et al. Applying Small Molecule Signal Transducer and Activator of Transcription-3 (STAT3) Protein Inhibitors as Pancreatic Cancer Therapeutics. Mol Cancer Ther15, 794-805, doi:10.1158/15357163.MCT-15-0003 (2016).

56 Lindblom, P. et al. Tesaglitazar, a dual PPAR-alpha/gamma agonist, hamster carcinogenicity, investigative animal and clinical studies. Toxicol Patho/40, 18-32, doi:10.1177/0192623311429972 (2012).

57 Ozcelikkale, A. et al. Differential response to doxorubicin in breast cancer subtypes simulated by a microfluidic tumor model. Journal of controlled release : official journal of the Controlled Release Society266, 129-139, doi:10.1016/j.jconrel.2017.09.024 (2017).

58 Kwak, B., Ozcelikkale, A., Shin, C. S., Park, K. \& Han, B. Simulation of complex transport of nanoparticles around a tumor using tumor-microenvironment-on-chip. Journal of controlled release : official journal of the Controlled Release Society194, 157-167, doi:10.1016/j.jconrel.2014.08.027 (2014).

59 Shin, C. S., Kwak, B., Han, B. \& Park, K. Development of an in vitro 3D tumor model to study therapeutic efficiency of an anticancer drug. Mol Pharm10, 2167-2175, doi:10.1021/mp300595a (2013).

60 Fitzmaurice, G. M., Laird, N. M. \& Ware, J. H. in Statistics in Medicine (Wlley Online Library, 2004). 
61 Baran-Gale, J., Chandra, T. \& Kirschner, K. Experimental design for single-cell RNA sequencing. Brief Funct Genomics17, 233-239, doi:10.1093/bfgp/elx035 (2018).

62 Ziegenhain, C. et al. Comparative Analysis of Single-Cell RNA Sequencing Methods. Mol Cel/65, 631643 e634, doi:10.1016/j.molcel.2017.01.023 (2017).

63 Zhang, Y. et al. M3S: a comprehensive model selection for multi-modal single-cell RNA sequencing data. BMC bioinformatics20, 672, doi:10.1186/s12859-019-3243-1 (2019).

64 Xie, J. et al. QUBIC2: a novel and robust biclustering algorithm for analyses and interpretation of largescale RNA-Seq data. Bioinformatics36, 1143-1149, doi:10.1093/bioinformatics/btz692 (2020).

65 Luo, M. et al. Role of the multifunctional DNA repair and redox signaling protein Ape1/Ref-1 in cancer and endothelial cells: small-molecule inhibition of the redox function of Ape1. Antioxid Redox Signa/10, 1853-1867, doi:10.1089/ars.2008.2120 (2008).

66 Kelley, M. R. et al. Functional Analysis of Novel Analogs of E3330 That Block the Redox Signaling Activity of the Multifunctional AP Endonuclease/Redox Signaling Enzyme APE1/Ref-1. Antioxid Redox Signa/14, 1387-1401, doi:10.1089/ars.2010.3410 (2011).

$67 \mathrm{Moon}, \mathrm{H}$. R. et al. An engineered pancreatic cancer model with intra-tumoral heterogeneity of driver mutations. Lab on a chip20, 3720-3732, doi:10.1039/d0lc00707b (2020).

68 Zhou, Y. et al. Intracellular ATP levels are a pivotal determinant of chemoresistance in colon cancer cells. Cancer Res72, 304-314, doi:10.1158/0008-5472.CAN-11-1674 (2012).

69 Viale, A. et al. Oncogene ablation-resistant pancreatic cancer cells depend on mitochondrial function. Nature514, 628-632, doi:10.1038/nature13611 (2014).

70 Alistar, A. et al. Safety and tolerability of the first-in-class agent CPI-613 in combination with modified FOLFIRINOX in patients with metastatic pancreatic cancer: a single-centre, open-label, dose-escalation, phase 1 trial. The Lancet Oncology18, 770-778, doi:10.1016/s1470-2045(17)30314-5 (2017).

71 Vascotto, C. et al. Genome-wide analysis and proteomic studies reveal APE1/Ref-1 multifunctional role in mammalian cells. Proteomics9, 1058-1074, doi:10.1002/pmic.200800638 (2009).

72 Illuzzi, J. L. et al. Tumor-associated APE1 variant exhibits reduced complementation efficiency but does not promote cancer cell phenotypes. Environ Mol Mutagen, doi:10.1002/em.22074 (2017).

73 Amanakis, G. \& Murphy, E. Cyclophilin D: An Integrator of Mitochondrial Function. Frontiers in physiology11, 595, doi:10.3389/fphys.2020.00595 (2020).

74 Chiu, H. Y., Tay, E. X. Y., Ong, D. S. T. \& Taneja, R. Mitochondrial Dysfunction at the Center of Cancer Therapy. Antioxid Redox Signa/32, 309-330, doi:10.1089/ars.2019.7898 (2020). 
75 Martinez-Outschoorn, U. E. et al. Energy transfer in "parasitic" cancer metabolism: mitochondria are the powerhouse and Achilles' heel of tumor cells. Cell Cycle10, 4208-4216, doi:10.4161/cc.10.24.18487 (2011).

76 Martinez-Outschoorn, U. E., Lisanti, M. P. \& Sotgia, F. Catabolic cancer-associated fibroblasts transfer energy and biomass to anabolic cancer cells, fueling tumor growth. Semin Cancer Bio/25, 47-60, doi:10.1016/j.semcancer.2014.01.005 (2014).

77 Martinez-Outschoorn, U. E. et al. Autophagy in cancer associated fibroblasts promotes tumor cell survival: Role of hypoxia, HIF1 induction and NFkappaB activation in the tumor stromal microenvironment. Cell Cycle9, 3515-3533, doi:10.4161/cc.9.17.12928 (2010).

78 Fiaschi, T. et al. Reciprocal metabolic reprogramming through lactate shuttle coordinately influences tumor-stroma interplay. Cancer Res72, 5130-5140, doi:10.1158/0008-5472.CAN-12-1949 (2012).

79 Rhim, A. D. et al. Stromal elements act to restrain, rather than support, pancreatic ductal adenocarcinoma. Cancer Cel/25, 735-747, doi:10.1016/j.ccr.2014.04.021 (2014).

80 Ozdemir, B. C. et al. Depletion of carcinoma-associated fibroblasts and fibrosis induces immunosuppression and accelerates pancreas cancer with reduced survival. Cancer Cel/25, 719-734, doi:10.1016/j.ccr.2014.04.005 (2014).

81 Jiang, H. et al. Pancreatic ductal adenocarcinoma progression is restrained by stromal matrix. J Clin Invest, doi:10.1172/JCl136760 (2020).

82 Chun, K. S., Jang, J. H. \& Kim, D. H. Perspectives Regarding the Intersections between STAT3 and Oxidative Metabolism in Cancer. Cells9, doi:10.3390/cells9102202 (2020).

83 Smolkova, K. et al. Nuclear Factor Erythroid 2-Related Factor 2 in Regulating Cancer Metabolism. Antioxid Redox Signa/33, 966-997, doi:10.1089/ars.2020.8024 (2020).

84 Bazzani, V. et al. Mitochondrial apurinic/apyrimidinic endonuclease 1 enhances mtDNA repair contributing to cell proliferation and mitochondrial integrity in early stages of hepatocellular carcinoma. BMC Cancer20, 969, doi:10.1186/s12885-020-07258-6 (2020).

85 Barchiesi, A., Wasilewski, M., Chacinska, A., Tell, G. \& Vascotto, C. Mitochondrial translocation of APE1 relies on the MIA pathway. Nucleic Acids Res43, 5451-5464, doi:10.1093/nar/gkv433 (2015).

86 Wang, X. et al. APE1/Ref-1 regulates 5-FU resistance in colon cancer cells through its redox and endonuclease activity. Int J Clin Exp Med12, 3870-3878 (2019).

\section{Figures}


A

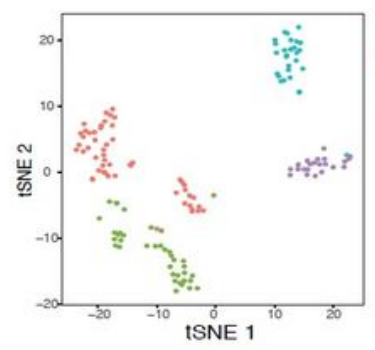

C

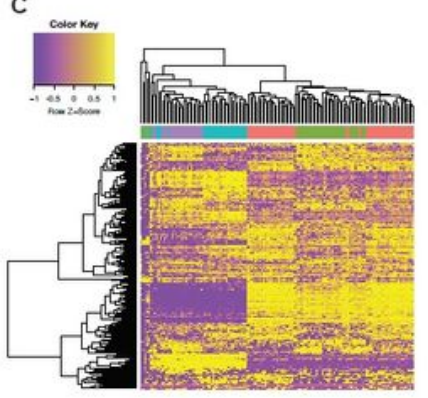

E

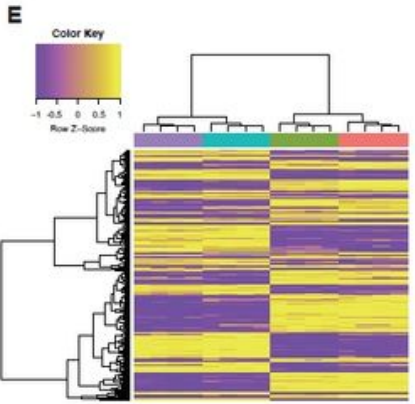

- Anowic cansol

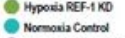
- Nomoria REF. 1 KO

B
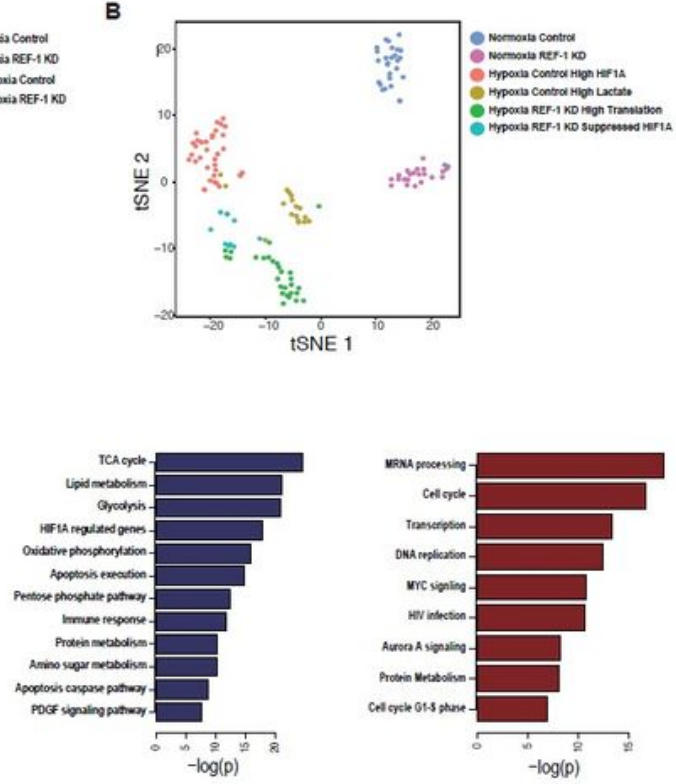

F
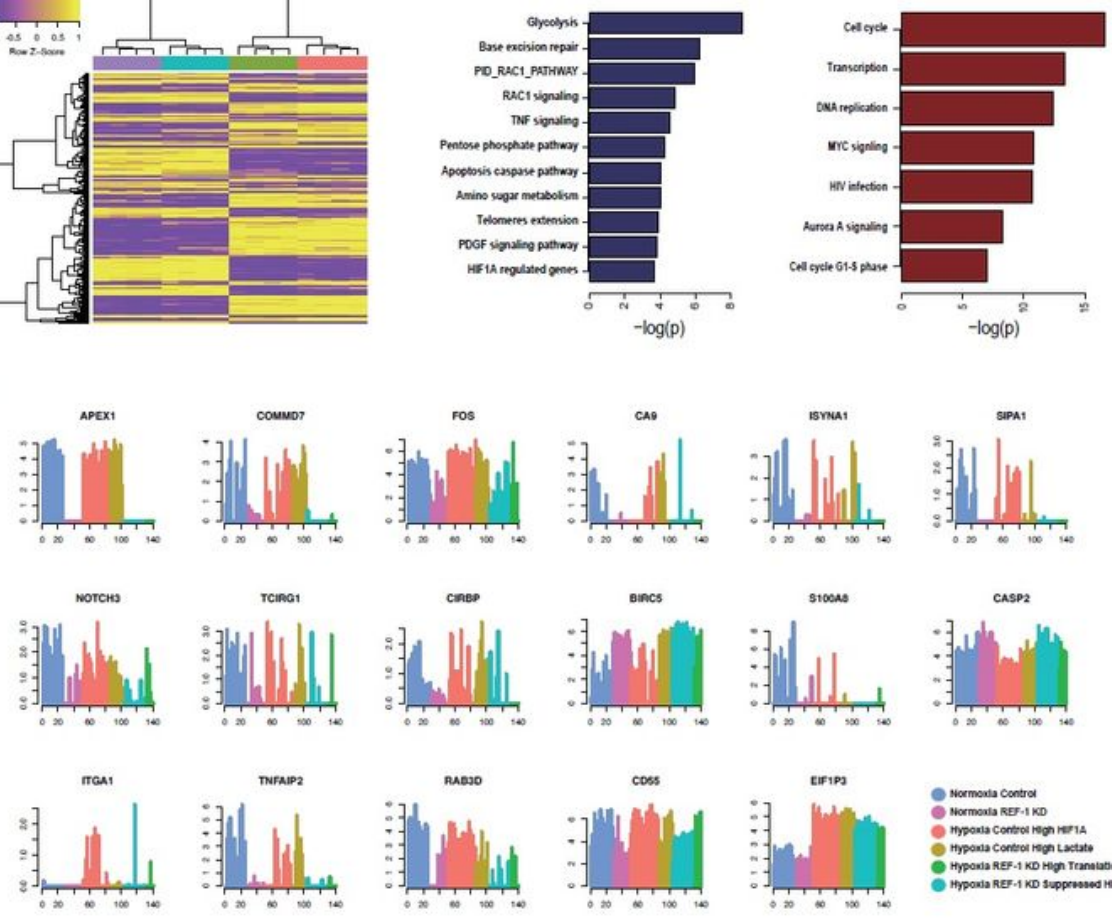

- Mermosta cantra

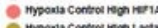

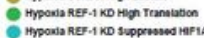

\section{Figure 1}

Integration of scRNA seq and proteomics following Ref- 1 downregulation under hypoxia. TSNE plot of the scRNA-seq data colored by experimental conditions $(A)$ and inferred cell clusters from unsupervised cell clustering (B). C. Heatmap of the top 250 genes with largest dispersion in the scRNA-seq data. High and low expression are colored by yellow and purple, respectively. The column color code represents the experimental condition of each cell annotated in (A). D. The top pathways in scRNA-seq data enriched by 
the down- (blue) and up- (red) regulated genes in Ref-1 KD vs Scr control under hypoxia. The x-axis is $\log$ (p.value) assessed by hypergeometric test. E. Heatmap of the proteomics data with the same color code as in C. F. The top pathways observed in the proteomics data enriched by the down- (blue) and up(red) regulated proteins in siRef-1 vs Scr control under hypoxia. G. Gene expression profile of selected genes with significant differential expression through the cell clusters. The color code is the same as the cell clusters annotated in (B).

A

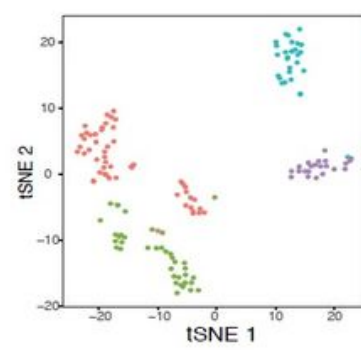

C

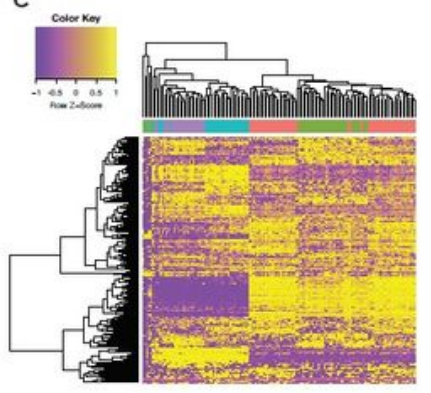

$\mathbf{E}$

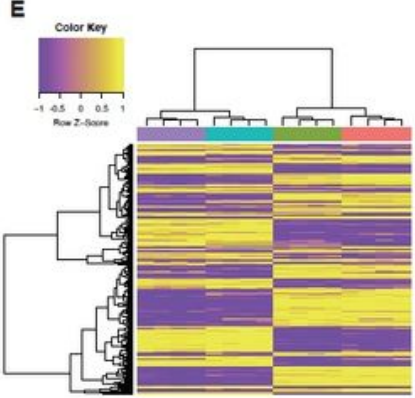

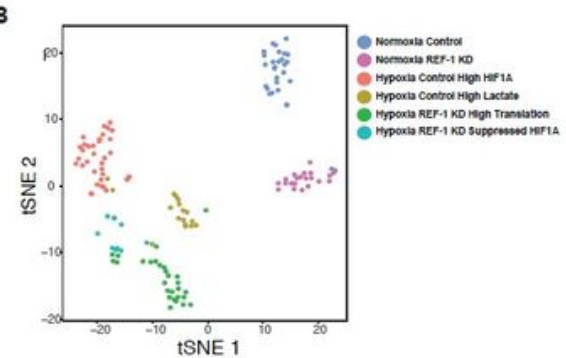

D

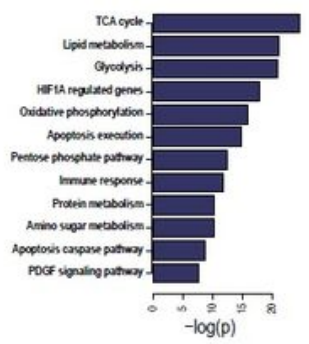

$\mathbf{F}$
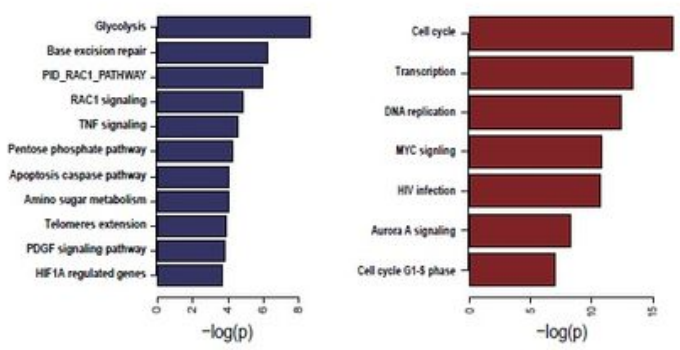

G
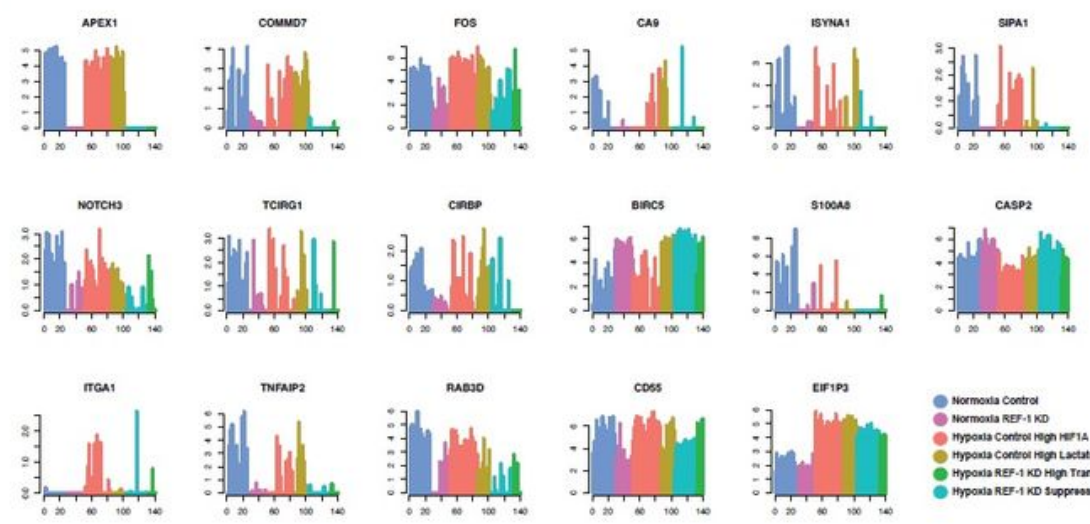

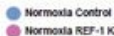

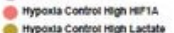

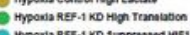

Figure 1 
Integration of scRNA seq and proteomics following Ref-1 downregulation under hypoxia. TSNE plot of the scRNA-seq data colored by experimental conditions $(A)$ and inferred cell clusters from unsupervised cell clustering (B). C. Heatmap of the top 250 genes with largest dispersion in the scRNA-seq data. High and low expression are colored by yellow and purple, respectively. The column color code represents the experimental condition of each cell annotated in (A). D. The top pathways in scRNA-seq data enriched by the down- (blue) and up- (red) regulated genes in Ref-1 KD vs Scr control under hypoxia. The x-axis is $\log (p . v a l u e)$ assessed by hypergeometric test. E. Heatmap of the proteomics data with the same color code as in C. F. The top pathways observed in the proteomics data enriched by the down- (blue) and up(red) regulated proteins in siRef-1 vs Scr control under hypoxia. G. Gene expression profile of selected genes with significant differential expression through the cell clusters. The color code is the same as the cell clusters annotated in (B). 
A

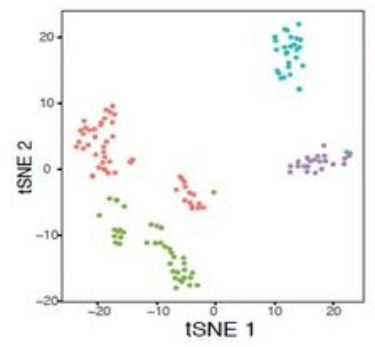

C
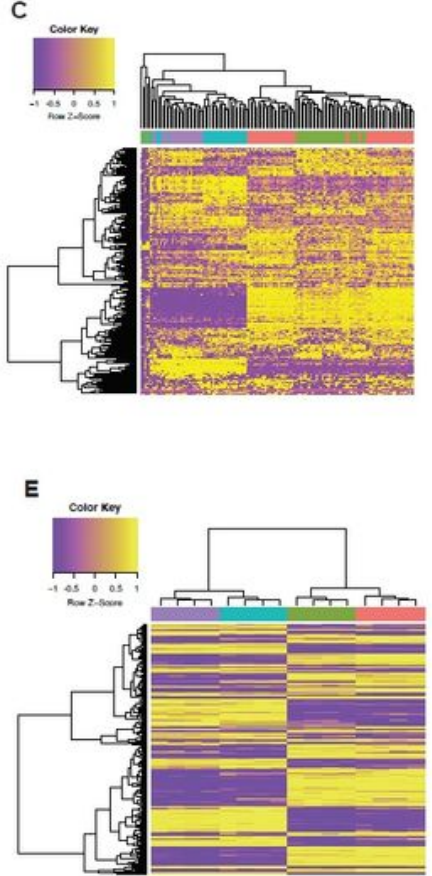

- Anposic canol

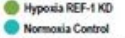
- Nomosid REF.1KO

B
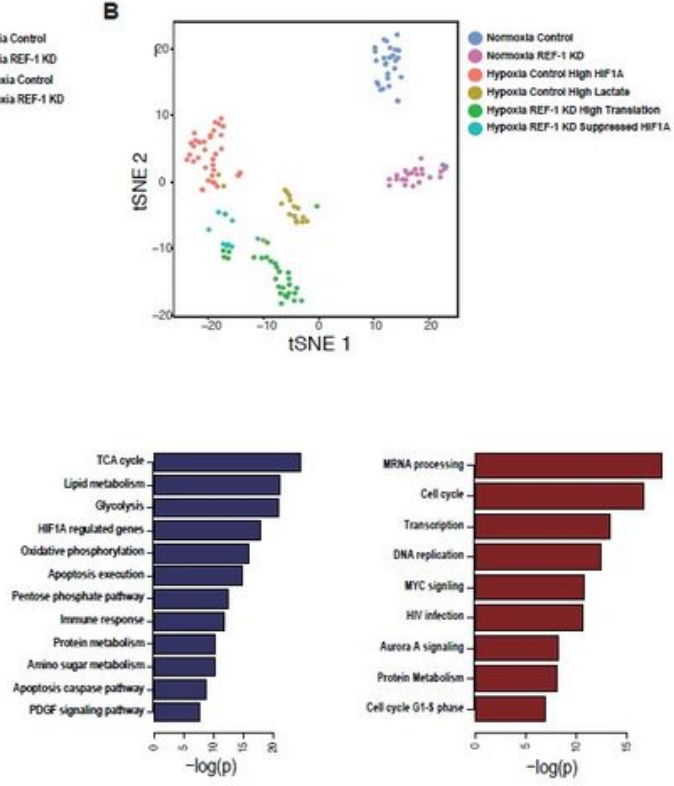

F
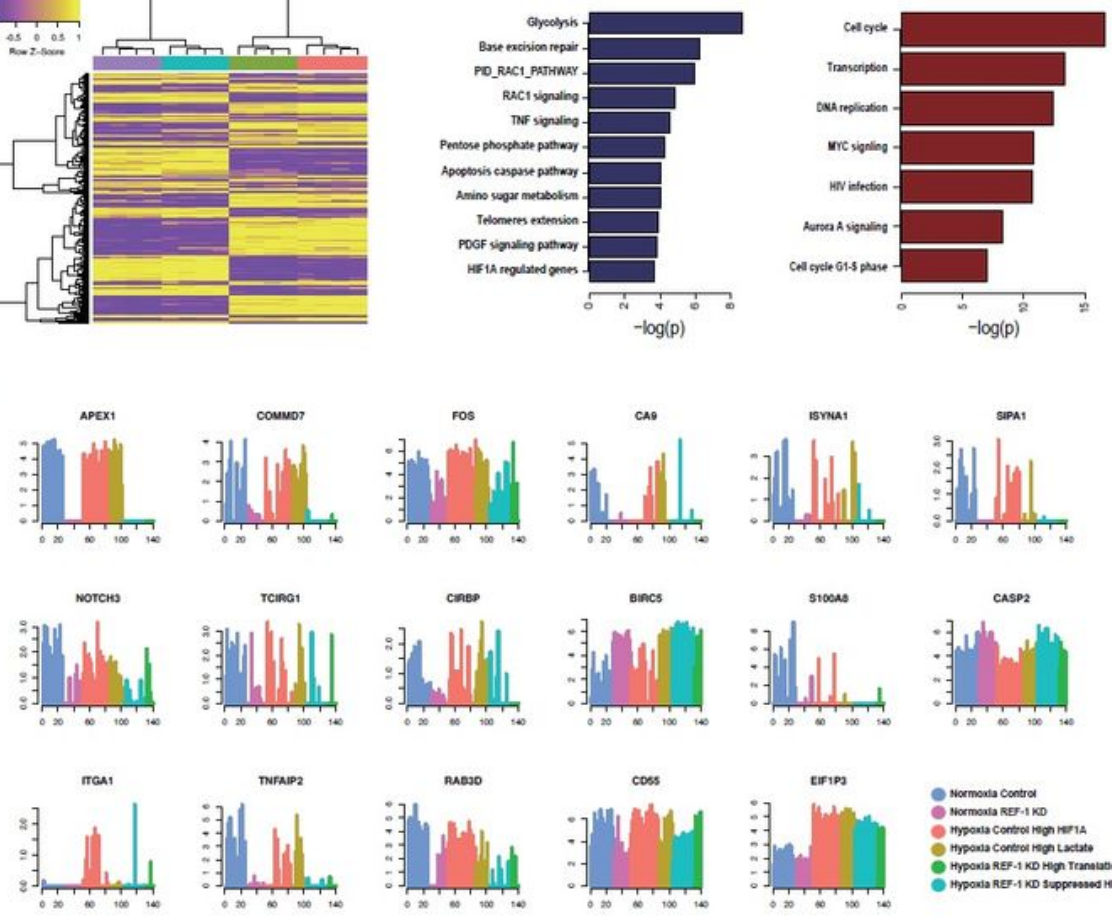

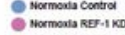

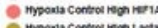

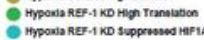

\section{Figure 1}

Integration of scRNA seq and proteomics following Ref- 1 downregulation under hypoxia. TSNE plot of the scRNA-seq data colored by experimental conditions $(A)$ and inferred cell clusters from unsupervised cell clustering (B). C. Heatmap of the top 250 genes with largest dispersion in the scRNA-seq data. High and low expression are colored by yellow and purple, respectively. The column color code represents the experimental condition of each cell annotated in (A). D. The top pathways in scRNA-seq data enriched by 
the down- (blue) and up- (red) regulated genes in Ref-1 KD vs Scr control under hypoxia. The x-axis is $\log$ (p.value) assessed by hypergeometric test. E. Heatmap of the proteomics data with the same color code as in C. F. The top pathways observed in the proteomics data enriched by the down- (blue) and up(red) regulated proteins in siRef-1 vs Scr control under hypoxia. G. Gene expression profile of selected genes with significant differential expression through the cell clusters. The color code is the same as the cell clusters annotated in (B).

A

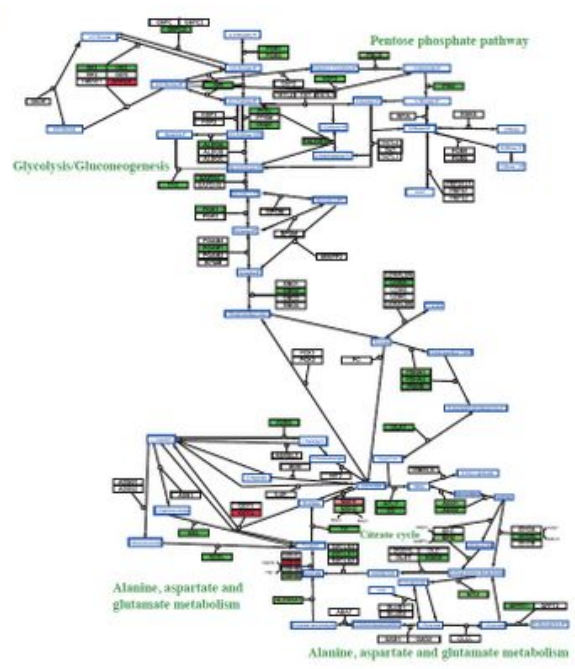

C

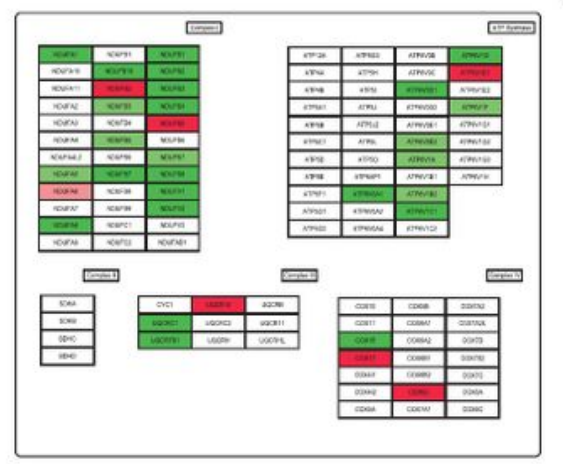

$\mathrm{E}$
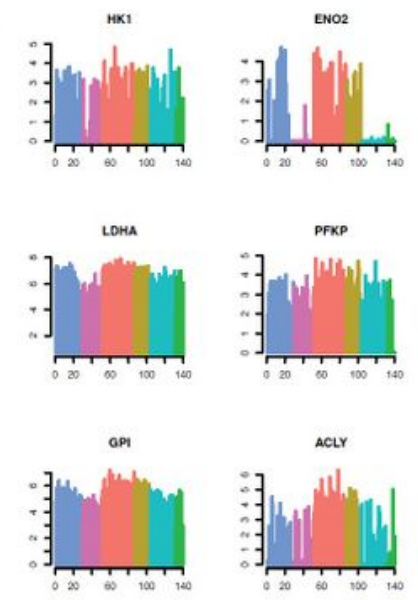
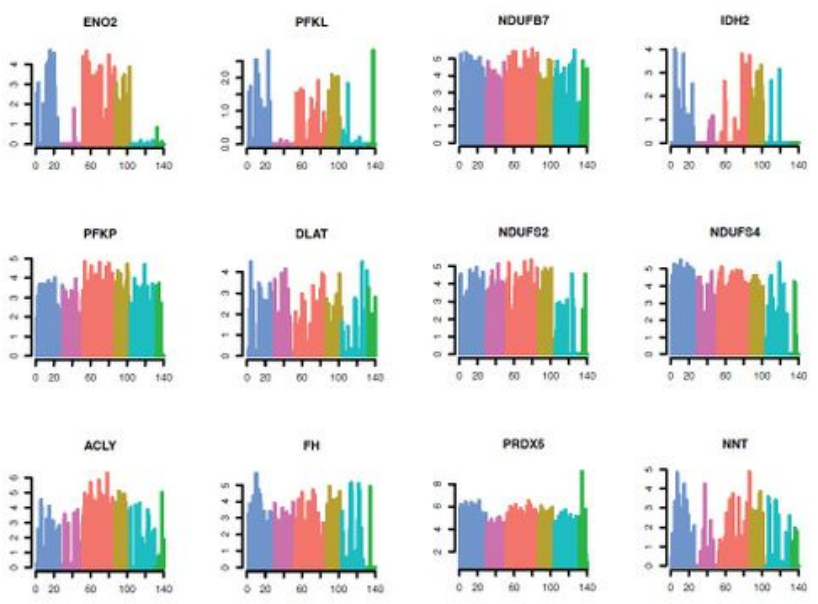

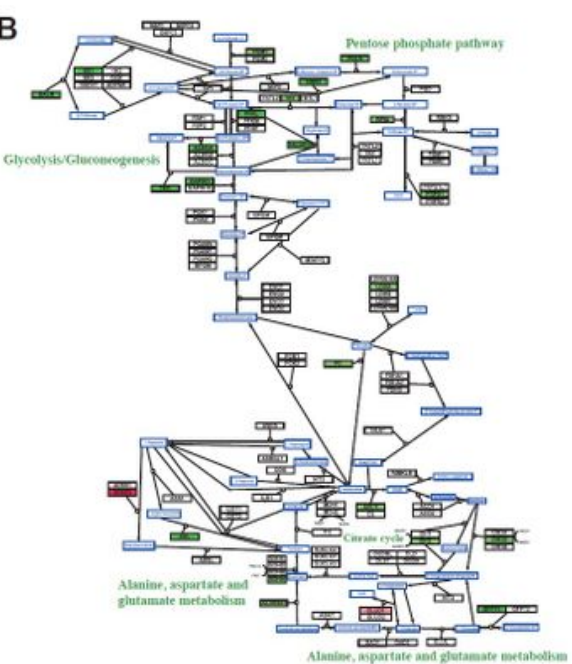

D

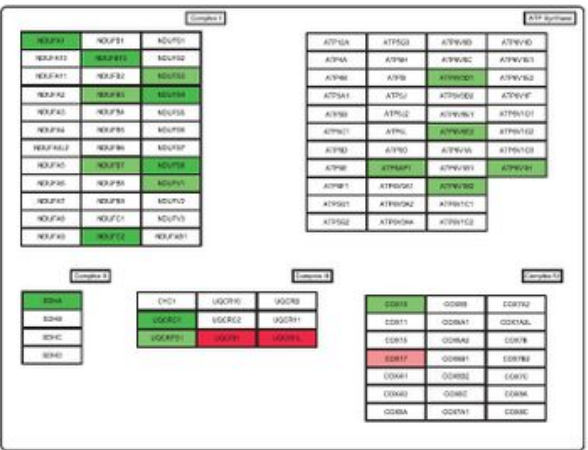

Nermoxia Controf
Nermoxia REF $-1 \mathrm{~K}$

Hypoxac Contol Hagh Hif $1 \mathrm{~A}$

Hyposes control hibh Lactate

Hyporta REE. 1 KO Hogh Tramsiston

Figure 2 
Blockade of Ref-1 under hypoxia causes downregulation of metabolic pathways. Differentially expressed central metabolic genes in siRef-1 vs Scr Control under hypoxia (A) and normoxia (B) conditions. The upand down- regulated genes were colored by light $(0.001<p<0.05)$ or dark $(p<0.001)$ red and green, respectively. Differentially expressed mitochondrial complex and ATP synthase genes in siRef-1 vs Scr control under hypoxia (C) and normoxia (D). E. Gene expression profile of key genes involved in the central metabolism through different cell groups. The color code is the same as Figure 1G.

A

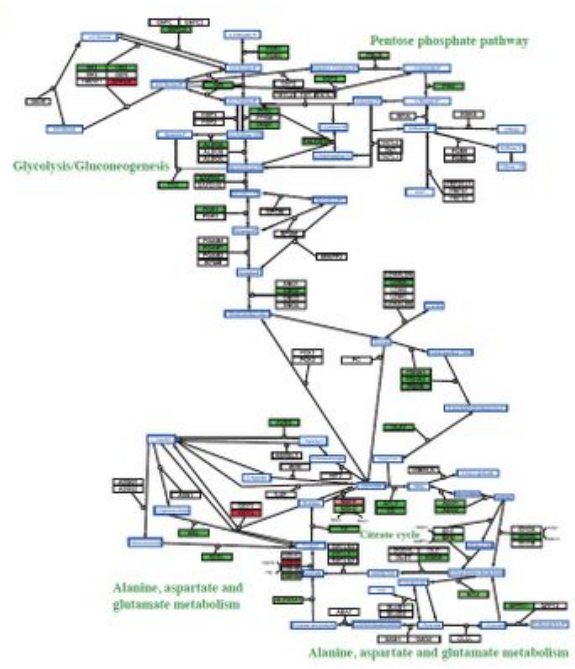

C

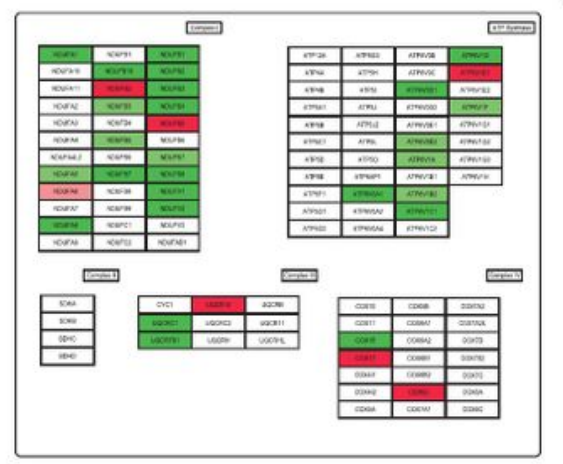

$\mathrm{E}$
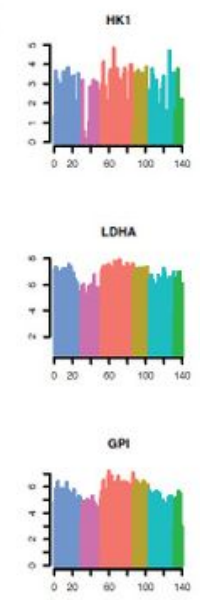
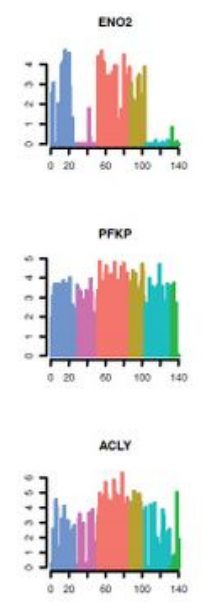
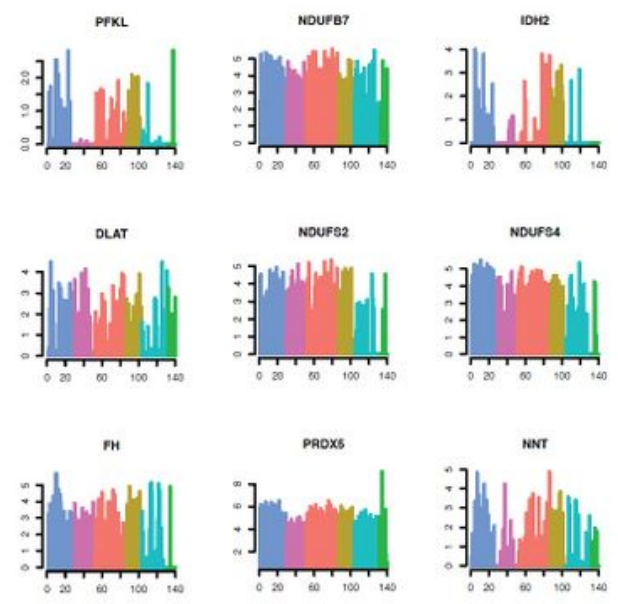
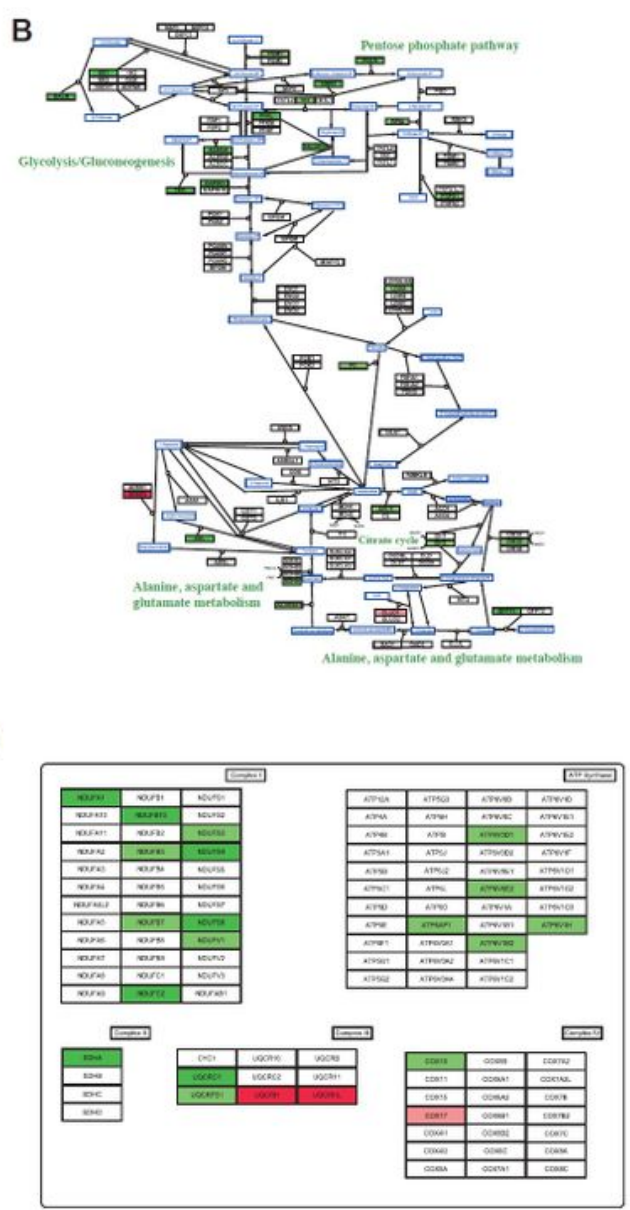
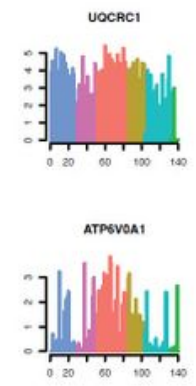

Nermoxia Consolot
Nermoxta REF $-1 \mathrm{KD}$ Hypoxala Control Hagh Hif $1 \mathrm{~A}$ Hypoxas control High Lactate Iyporta REE. 1 KO HSgh Tramsition 
Blockade of Ref-1 under hypoxia causes downregulation of metabolic pathways. Differentially expressed central metabolic genes in siRef-1 vs Scr Control under hypoxia (A) and normoxia (B) conditions. The upand down- regulated genes were colored by light $(0.001<p<0.05)$ or dark $(p<0.001)$ red and green, respectively. Differentially expressed mitochondrial complex and ATP synthase genes in siRef-1 vs Scr control under hypoxia (C) and normoxia (D). E. Gene expression profile of key genes involved in the central metabolism through different cell groups. The color code is the same as Figure 1G.

A

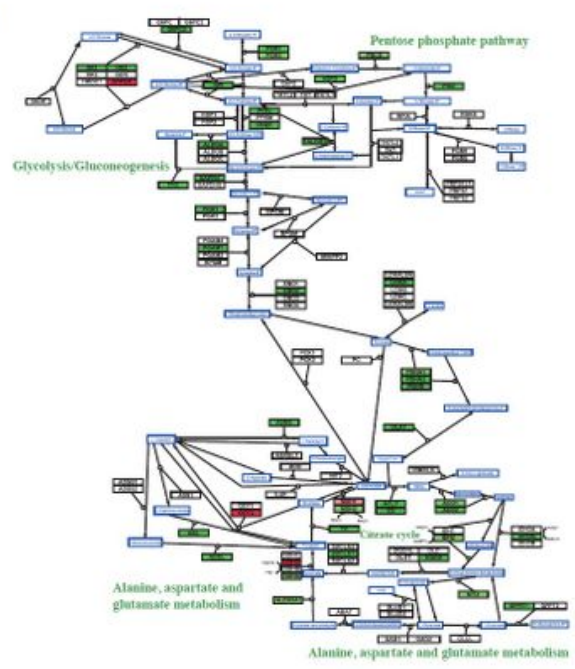

C

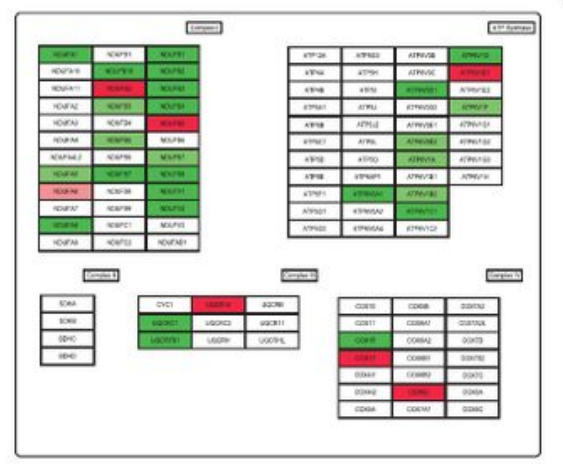

$\mathrm{E}$
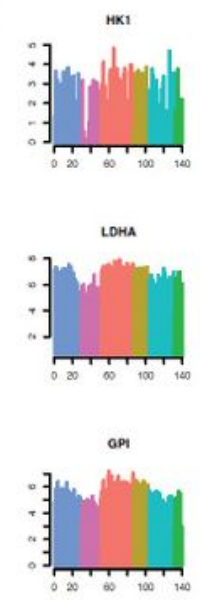
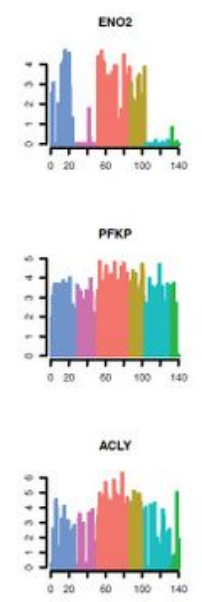
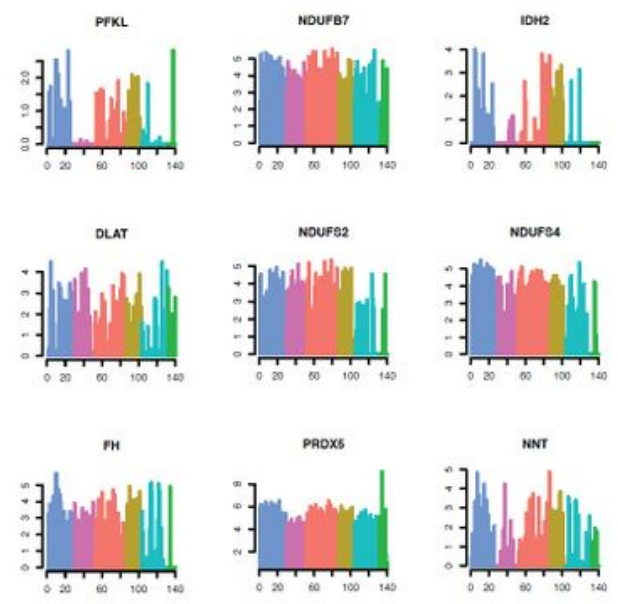
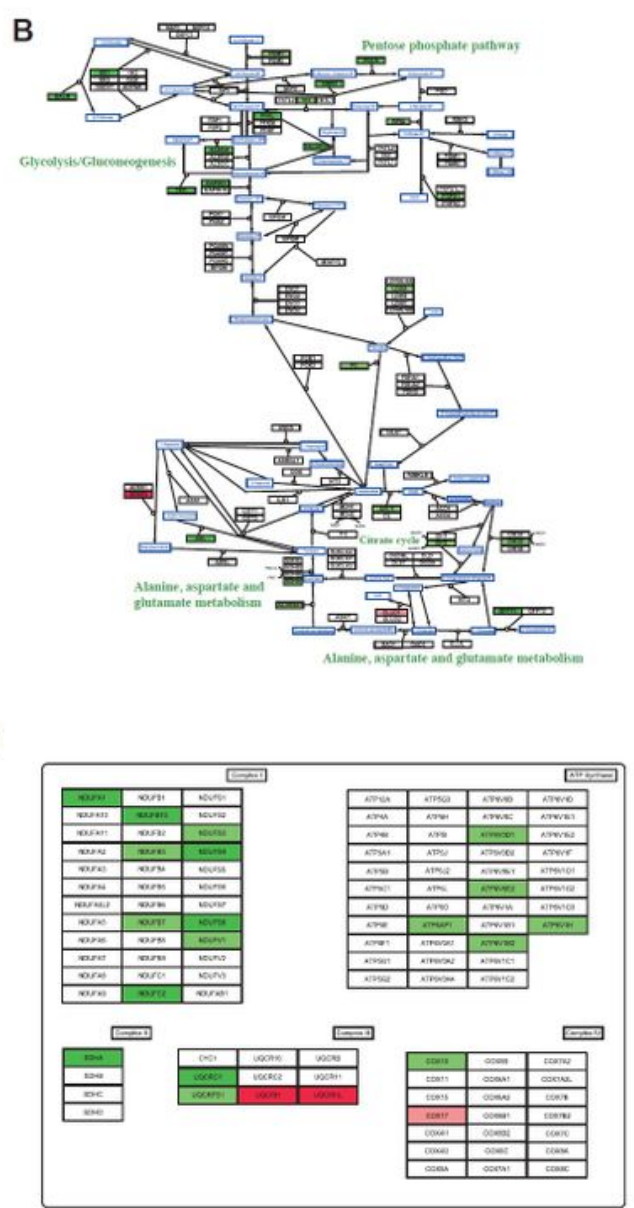
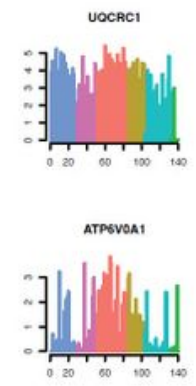

Nermoxia Consolot
Nermoxta REF $-1 \mathrm{KD}$ Hypoxala Control Hagh Hif $1 \mathrm{~A}$ Hypoxas control High Lactate Iyporta REE. 1 KO HSgh Tramsition 
Blockade of Ref-1 under hypoxia causes downregulation of metabolic pathways. Differentially expressed central metabolic genes in siRef-1 vs Scr Control under hypoxia (A) and normoxia (B) conditions. The upand down- regulated genes were colored by light $(0.001<p<0.05)$ or dark $(p<0.001)$ red and green, respectively. Differentially expressed mitochondrial complex and ATP synthase genes in siRef-1 vs Scr control under hypoxia (C) and normoxia (D). E. Gene expression profile of key genes involved in the central metabolism through different cell groups. The color code is the same as Figure 1G.
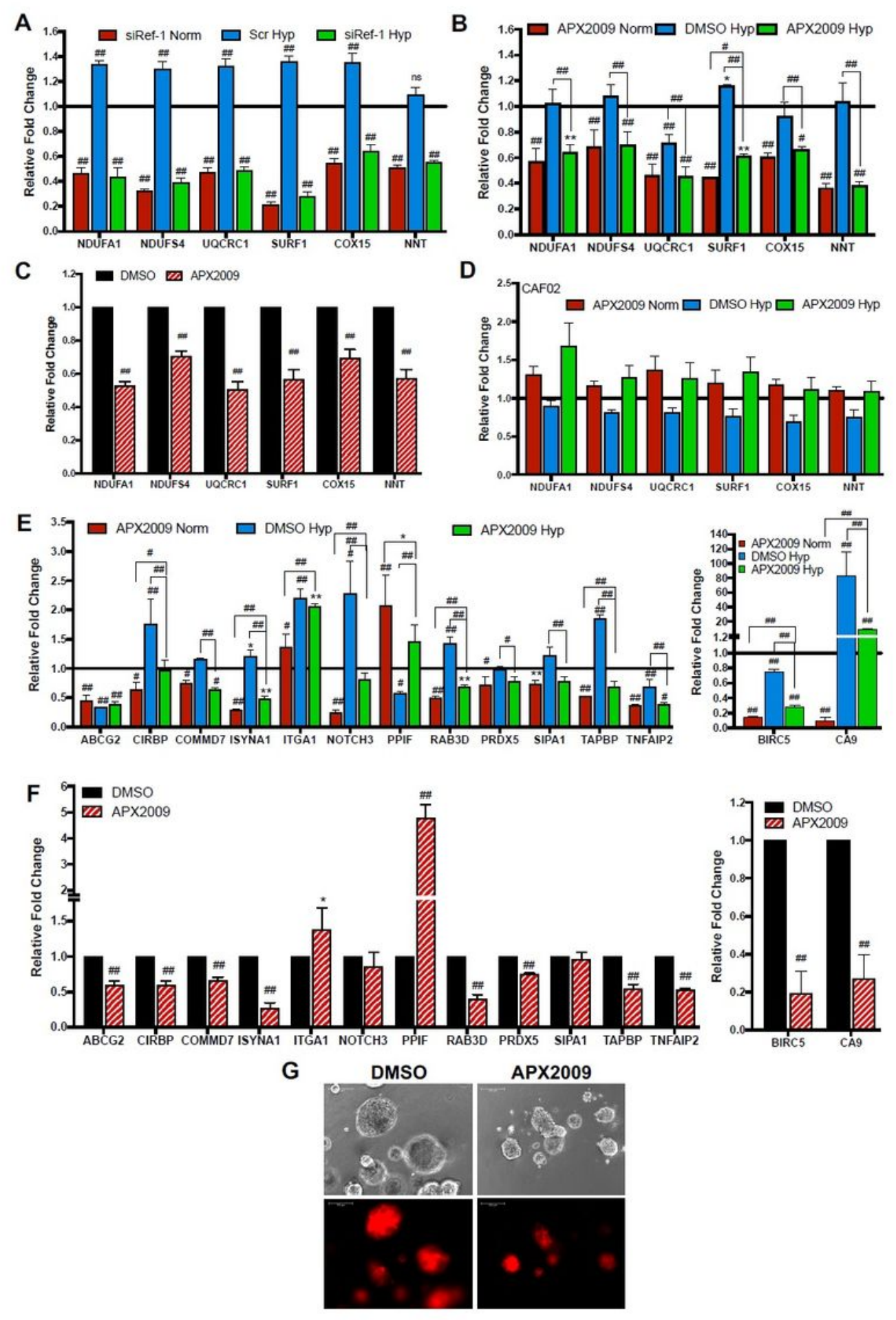

Figure 3 
Ref-1 inhibition downregulates mitochondrial complex genes as well as Ref-1 PD marker genes. A. Validation of selected mitochondrial complex genes from the scRNA-seq data using qRT-PCR (Scr/siRef-1 - 10nM, 1\% hypoxia for $24 \mathrm{~h}, \mathrm{n}=3, \mathrm{p}<0.0001$ ). Expression of mitochondrial complex genes (B) and Ref-1 regulated genes $(E)$ after treatment with Ref-1 redox inhibitor, APX2009 at $10 \mu \mathrm{M}$ under hypoxia $(1 \% 02)$ in Pa03C cells $(n=2, p<0.05-0.0001)$. D. Mitochondrial complex gene expression following Ref- 1 redox inhibition and hypoxia in CAFs. Mitochondrial complex gene panel (C) and Ref-1 regulated gene panel (F) after treatment with Ref-1 redox inhibitor (APX2009, 5 $\mu$ M) compared to vehicle control (DMSO) in Pa03C 3D spheroids ( $n=3, p<0.05-0.0001)$. BIRC5 (survivin) and CA9 were used as positive controls for Ref- 1 inhibition and hypoxia induction. G. Images representing the spheroids of $\mathrm{Pa} 03 \mathrm{C}$ cells used in the gene expression in Panels $C$ and F. (Scale Bar $-100 \mu \mathrm{m}, 10 \mathrm{X}$ mag). Relative fold change refers to the gene expression changes when compared to normoxia Scr or vehicle-treated cells. 

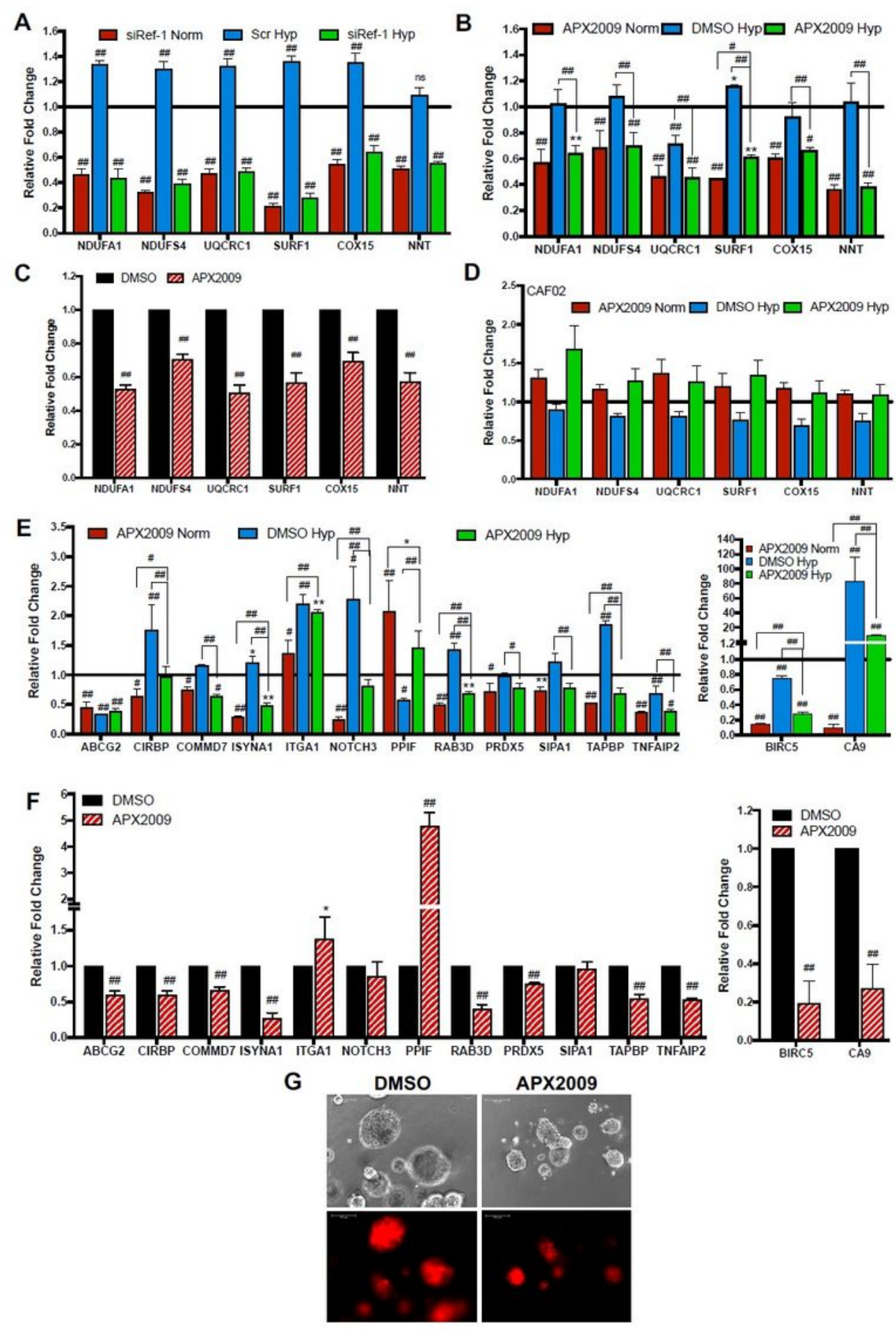

\section{Figure 3}

Ref-1 inhibition downregulates mitochondrial complex genes as well as Ref-1 PD marker genes. A. Validation of selected mitochondrial complex genes from the scRNA-seq data using qRT-PCR (Scr/siRef-1 - 10nM, 1\% hypoxia for $24 \mathrm{~h}, \mathrm{n}=3, \mathrm{p}<0.0001$ ). Expression of mitochondrial complex genes (B) and Ref-1 regulated genes $(E)$ after treatment with Ref-1 redox inhibitor, APX2009 at 10 $\mu \mathrm{M}$ under hypoxia $(1 \% 02)$ in Pa03C cells $(n=2, p<0.05-0.0001)$. D. Mitochondrial complex gene expression following Ref-1 redox 
inhibition and hypoxia in CAFs. Mitochondrial complex gene panel $(C)$ and Ref- 1 regulated gene panel $(F)$ after treatment with Ref-1 redox inhibitor (APX2009, 5 $\mu \mathrm{M}$ ) compared to vehicle control (DMSO) in Pa03C 3D spheroids ( $n=3, p<0.05-0.0001)$. BIRC5 (survivin) and CA9 were used as positive controls for Ref-1 inhibition and hypoxia induction. G. Images representing the spheroids of $\mathrm{Pa} 03 \mathrm{C}$ cells used in the gene expression in Panels $C$ and F. (Scale Bar $-100 \mu \mathrm{m}, 10 \mathrm{X}$ mag). Relative fold change refers to the gene expression changes when compared to normoxia Scr or vehicle-treated cells.
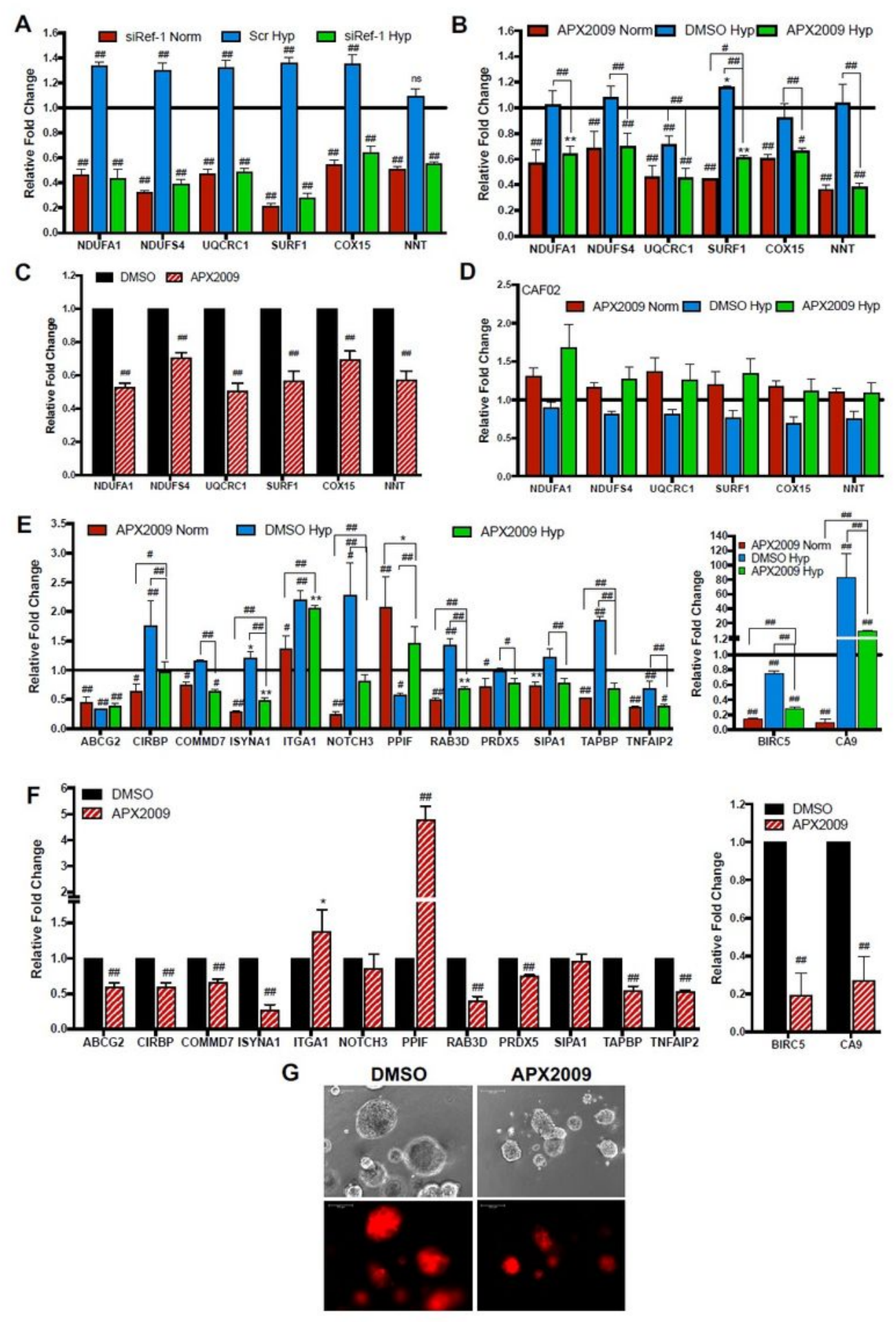

Figure 3 
Ref-1 inhibition downregulates mitochondrial complex genes as well as Ref-1 PD marker genes. A. Validation of selected mitochondrial complex genes from the scRNA-seq data using qRT-PCR (Scr/siRef-1 - 10nM, 1\% hypoxia for $24 \mathrm{~h}, \mathrm{n}=3, \mathrm{p}<0.0001$ ). Expression of mitochondrial complex genes (B) and Ref-1 regulated genes $(E)$ after treatment with Ref-1 redox inhibitor, APX2009 at $10 \mu \mathrm{M}$ under hypoxia $(1 \% 02)$ in Pa03C cells $(n=2, p<0.05-0.0001)$. D. Mitochondrial complex gene expression following Ref- 1 redox inhibition and hypoxia in CAFs. Mitochondrial complex gene panel (C) and Ref-1 regulated gene panel (F) after treatment with Ref-1 redox inhibitor (APX2009, 5 $\mu$ M) compared to vehicle control (DMSO) in Pa03C 3D spheroids ( $n=3, p<0.05-0.0001)$. BIRC5 (survivin) and CA9 were used as positive controls for Ref- 1 inhibition and hypoxia induction. G. Images representing the spheroids of $\mathrm{Pa} 03 \mathrm{C}$ cells used in the gene expression in Panels $C$ and F. (Scale Bar $-100 \mu \mathrm{m}, 10 \mathrm{X}$ mag). Relative fold change refers to the gene expression changes when compared to normoxia Scr or vehicle-treated cells. 

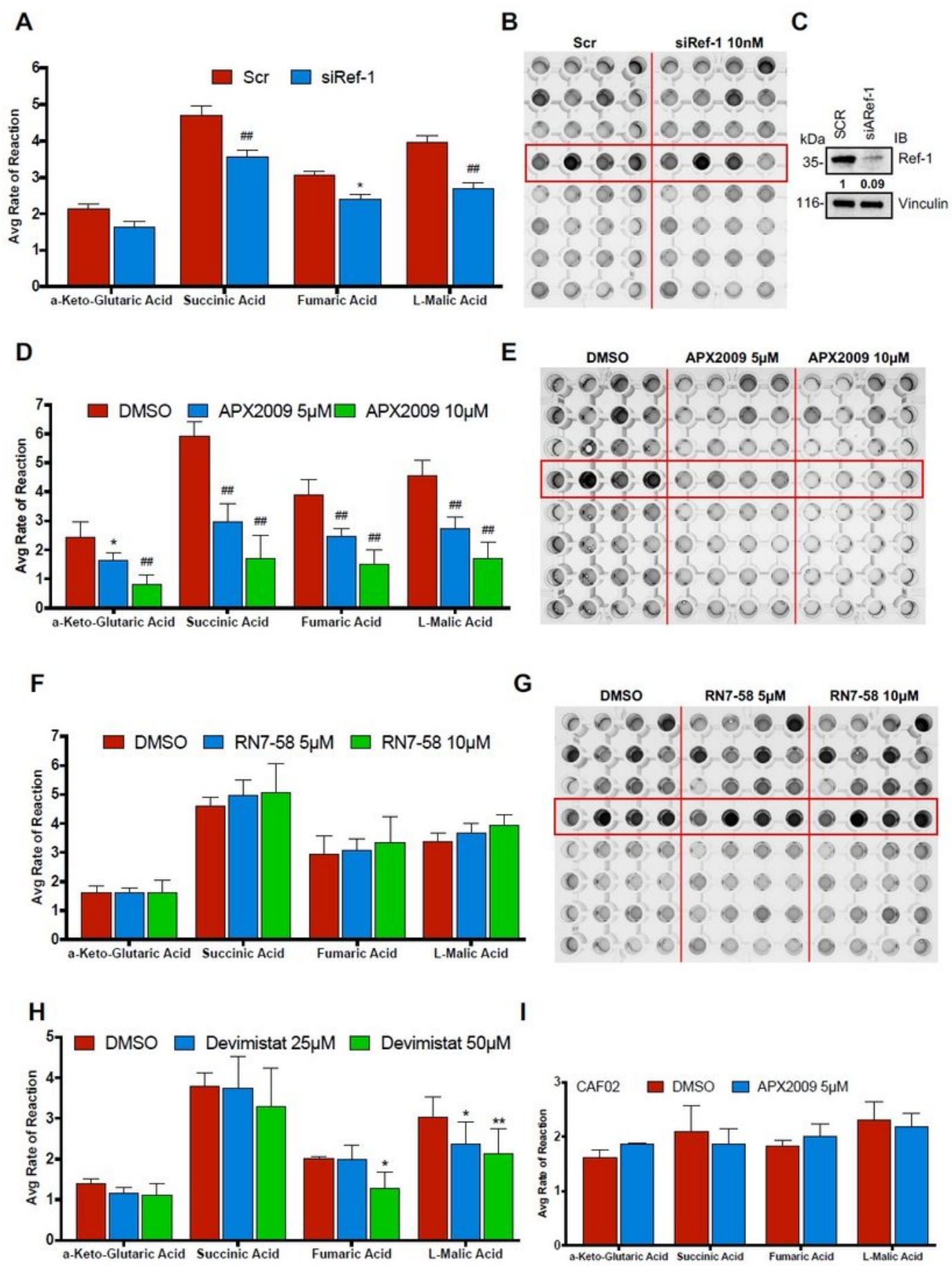

\section{Figure 4}

Ref-1 genetic or pharmacological inhibition reduces TCA cycle substrates. Mitochondrial functional assays in Pa03C cells transfected with Scr vs $10 n M$ siRef-1 (A, $\left.n=3,{ }^{*}<<0.05, \# \# 0.0001\right)$ and a representative image of the plate (B). Avg rate of reaction refers to slope of absorbance at 590 vs time. Western blot image of the Pa03C cells after transfection with Ref-1 or Scr siRNA (C). Vinculin is used as the loading control. Average rate of reaction in Pa03C cells treated with Ref- 1 redox inhibitor for $24 \mathrm{~h}$ (D, 
$\mathrm{n}=3$, * $\mathrm{p}<0.05, \# \# 0.0001)$, inactive Ref-1 redox inhibitor analog (F, RN7-58, n=2), metabolic inhibitor Devimistat $\left(H, n=3,{ }^{*} p<0.05,{ }^{*} 0.01\right)$ and representative plate images $(E, G)$. I. Average rate of reaction in CAF02 cells treated with $5 \mu$ M APX2009 for $24 \mathrm{~h}(\mathrm{n}=3)$. All data represent Mean \pm SE.
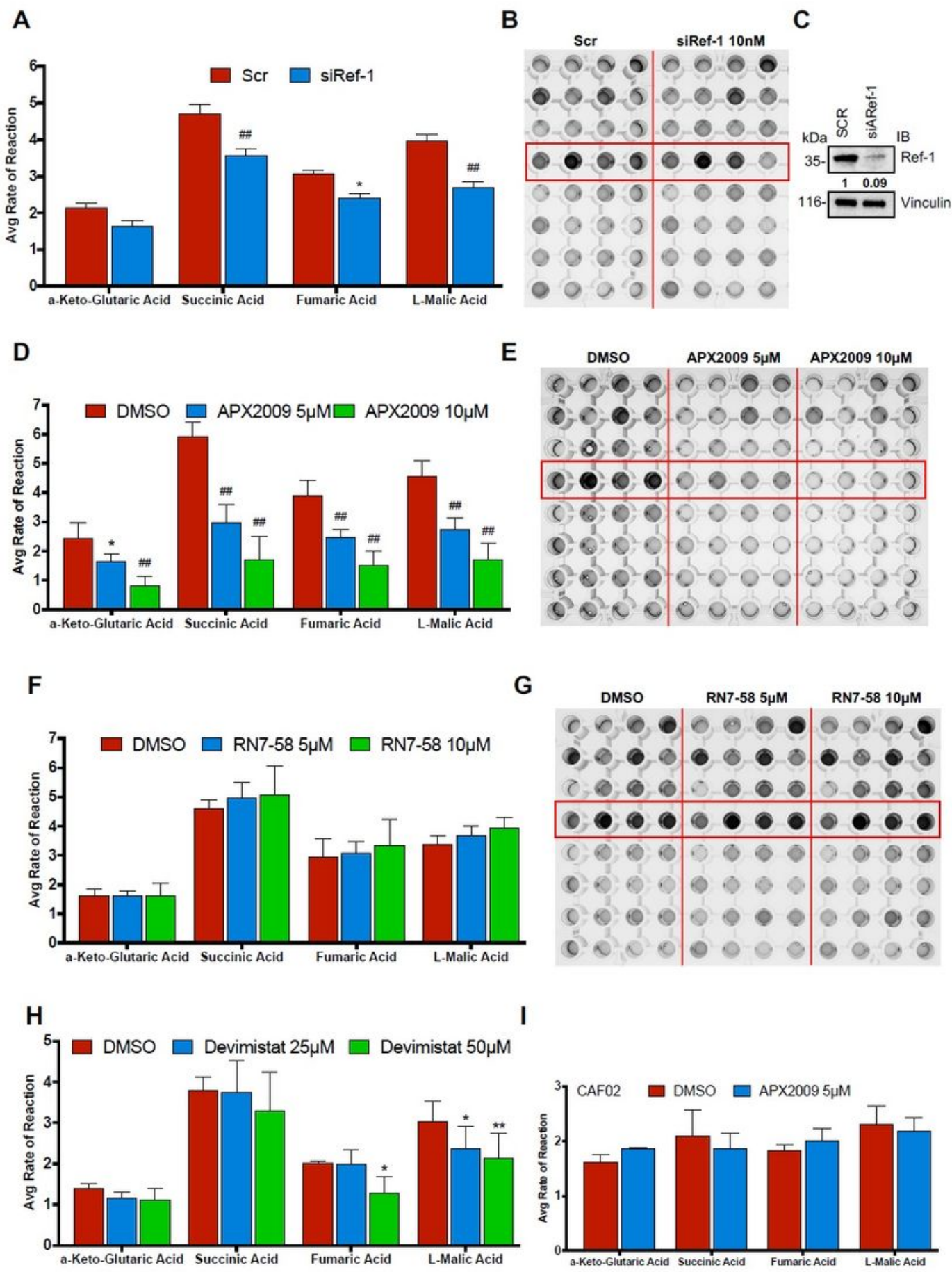

Figure 4

Ref-1 genetic or pharmacological inhibition reduces TCA cycle substrates. Mitochondrial functional assays in Pa03C cells transfected with Scr vs 10nM siRef-1 ( $\left.A, n=3,{ }^{*} p<0.05, \# \# 0.0001\right)$ and a 
representative image of the plate (B). Avg rate of reaction refers to slope of absorbance at 590 vs time. Western blot image of the Pa03C cells after transfection with Ref-1 or Scr siRNA (C). Vinculin is used as the loading control. Average rate of reaction in Pa03C cells treated with Ref- 1 redox inhibitor for $24 \mathrm{~h}(\mathrm{D}$, $\mathrm{n}=3, * \mathrm{p}<0.05, \# \# 0.0001)$, inactive Ref-1 redox inhibitor analog ( $F, R N 7-58, \mathrm{n}=2)$, metabolic inhibitor Devimistat $\left(H, n=3,{ }^{*} p<0.05, * \star 0.01\right)$ and representative plate images $(E, G)$. I. Average rate of reaction in CAF02 cells treated with $5 \mu$ M APX2009 for $24 \mathrm{~h}(n=3)$. All data represent Mean \pm SE.
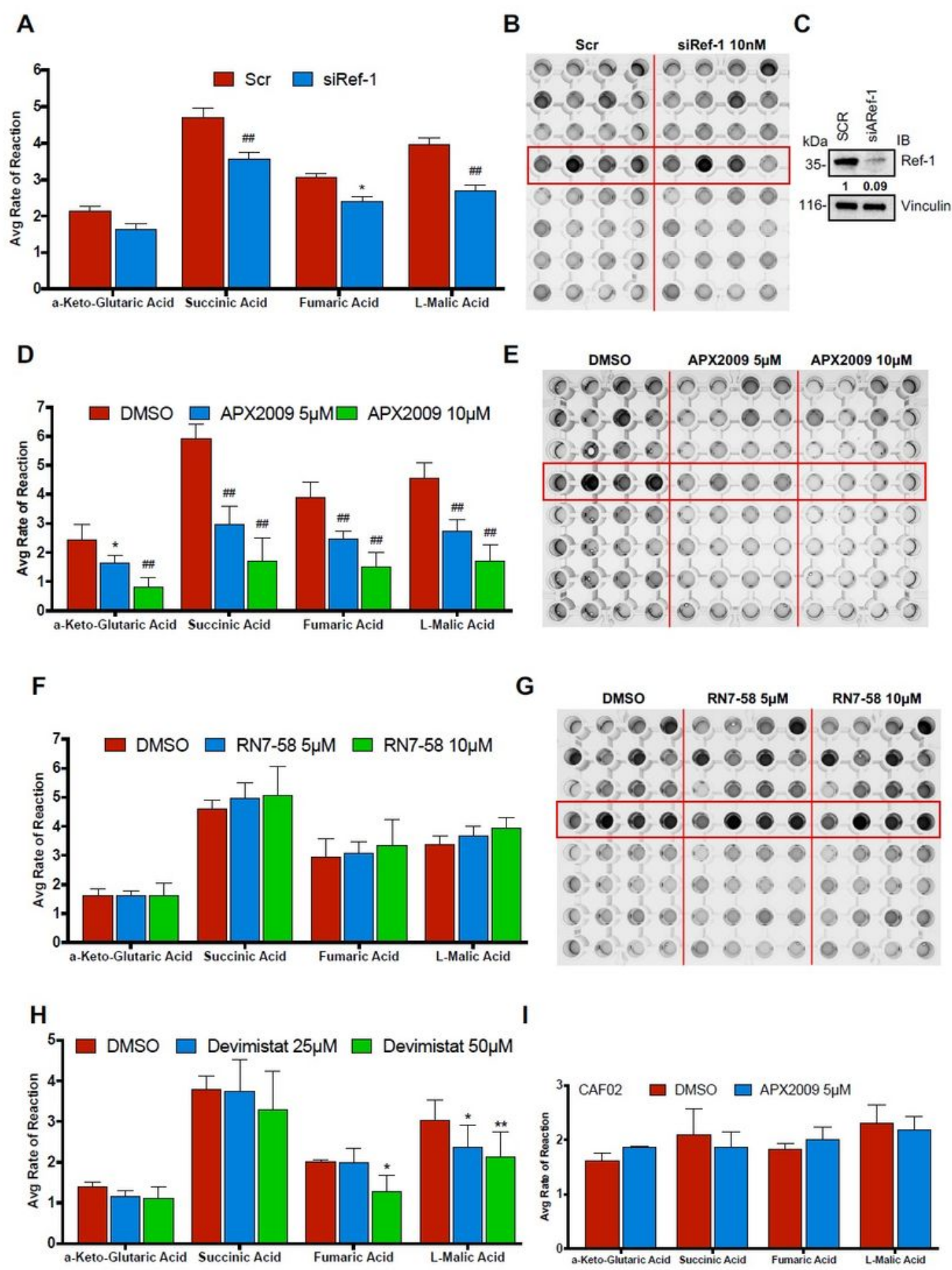

Figure 4 
Ref-1 genetic or pharmacological inhibition reduces TCA cycle substrates. Mitochondrial functional assays in Pa03C cells transfected with Scr vs $10 n M$ siRef-1 (A, $\left.n=3,{ }^{*} p<0.05, \# \# 0.0001\right)$ and a representative image of the plate (B). Avg rate of reaction refers to slope of absorbance at 590 vs time. Western blot image of the Pa03C cells after transfection with Ref-1 or Scr siRNA (C). Vinculin is used as the loading control. Average rate of reaction in Pa03C cells treated with Ref-1 redox inhibitor for $24 \mathrm{~h}$ ( $D$, $n=3, * p<0.05, \# \# 0.0001)$, inactive Ref-1 redox inhibitor analog ( $F, R N 7-58, n=2)$, metabolic inhibitor Devimistat $\left(H, n=3,{ }^{\star} p<0.05,{ }^{*} 0.01\right)$ and representative plate images $(E, G)$. I. Average rate of reaction in CAF02 cells treated with $5 \mu$ M APX2009 for $24 \mathrm{~h}(n=3)$. All data represent Mean \pm SE. 
A

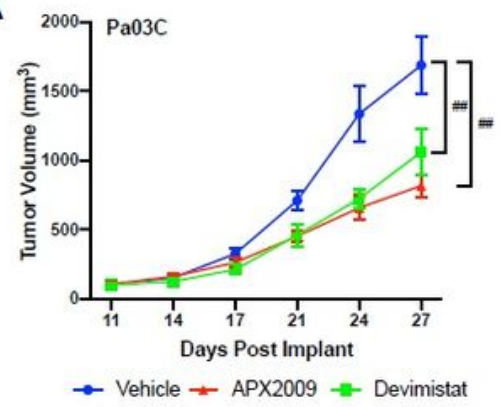

D ${ }^{1000} 7^{10.05+C A F 19}$

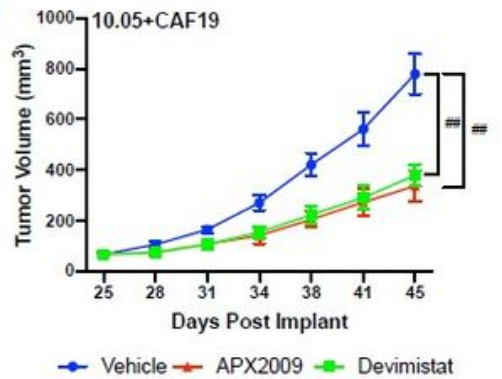

B

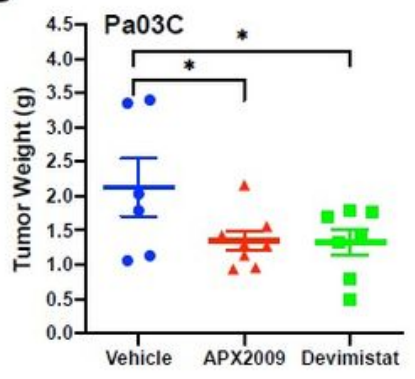

E

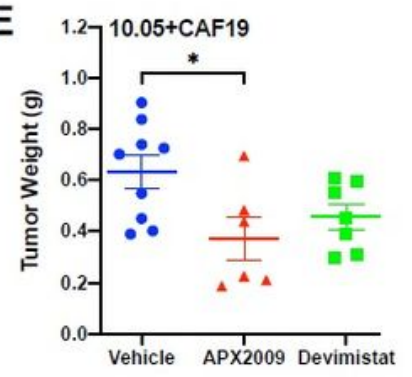

C

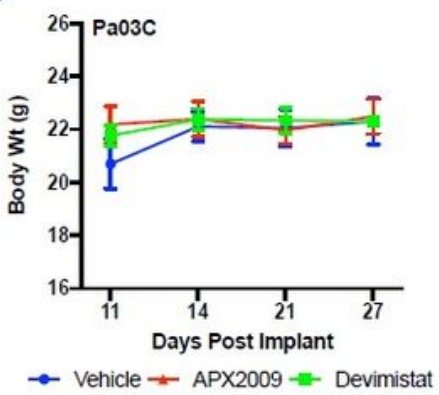

$\mathbf{F}$

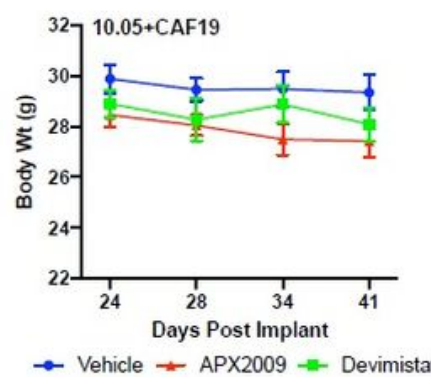

G

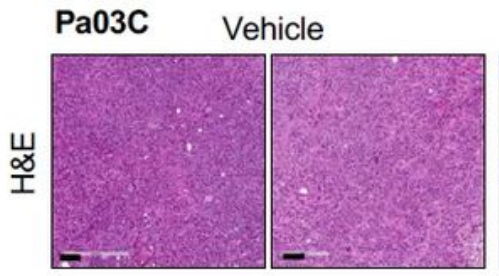

APX2009

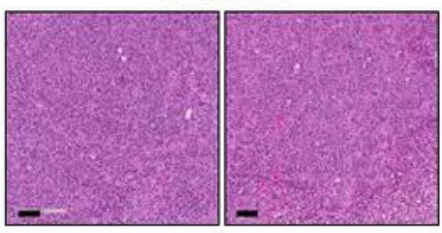

Devimistat

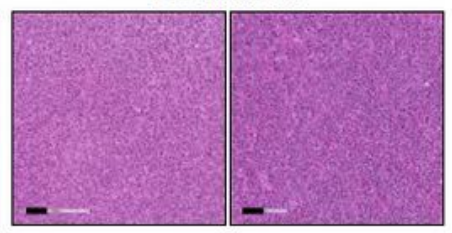

$10.05+$ CAF19
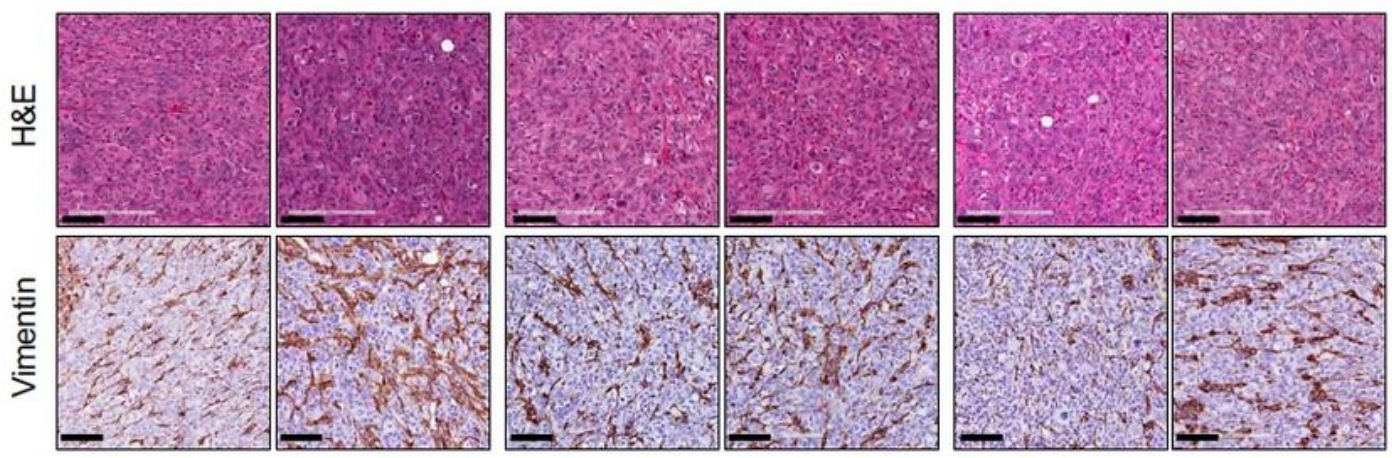

\section{Figure 5}

Ref-1 inhibition with APX2009 results in diminished tumor growth. Tumor growth of Pa03C (A) or Panc10.05+CAF19 (D) subcutaneous xenografts treated with PKT Vehicle or $35 \mathrm{mg} / \mathrm{Kg}$ APX2009 or $50 \mathrm{mg} / \mathrm{Kg}$ Devimistat (\#\#p<0.0001, compared to vehicle control) twice a day, $8 \mathrm{~h}$ apart, continuously for 15 (for Pa03C xenografts) or 20 (for Panc10.05+CAF19 xenografts) days. And corresponding tumor weights $\left(B, E,{ }^{*}<<0.05\right.$, compared to vehicle control). C\&F. Graphs representing body weights over time for 

positivity for Pa03C tumors and Panc10.05+CAF19 (G). All data are represented as Mean \pm SE.

A
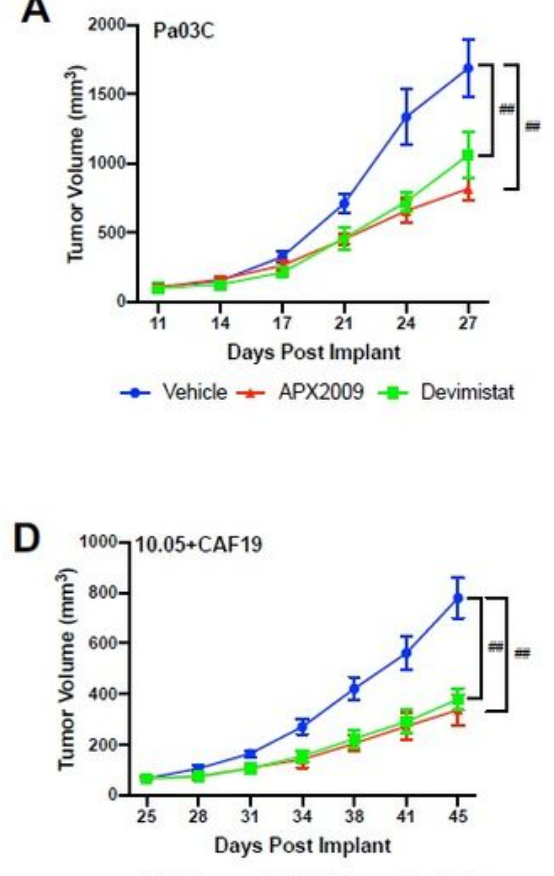

$\rightarrow$ Vehicle $\rightarrow$ APX2009 - Devimistat

G
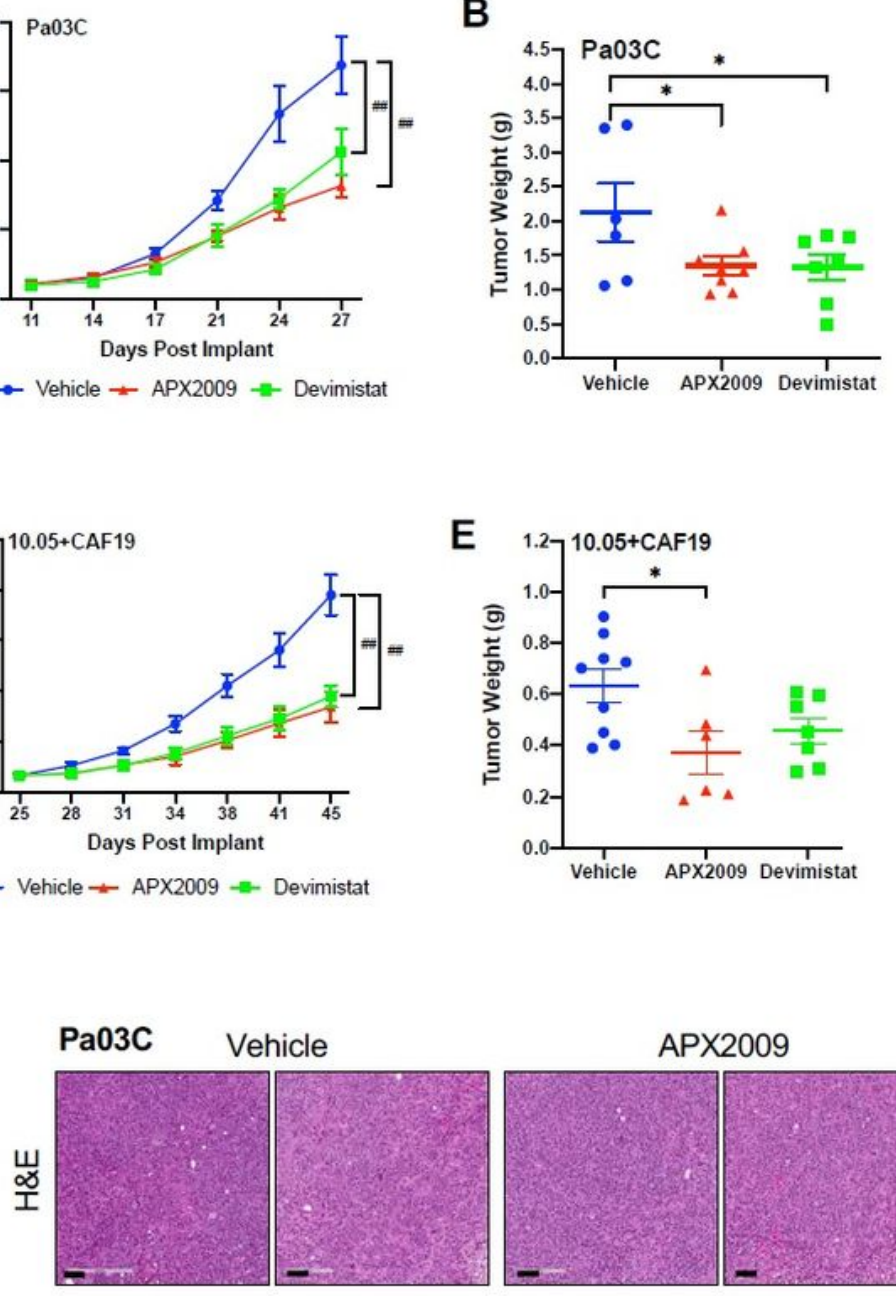

C

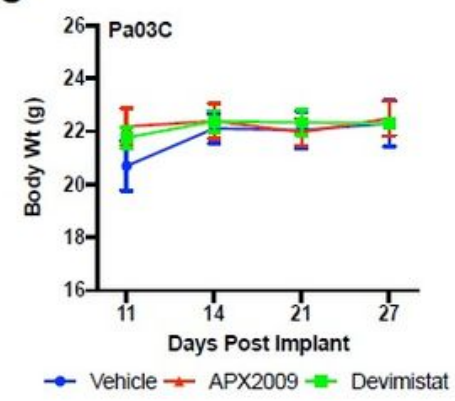

$\mathbf{F}$
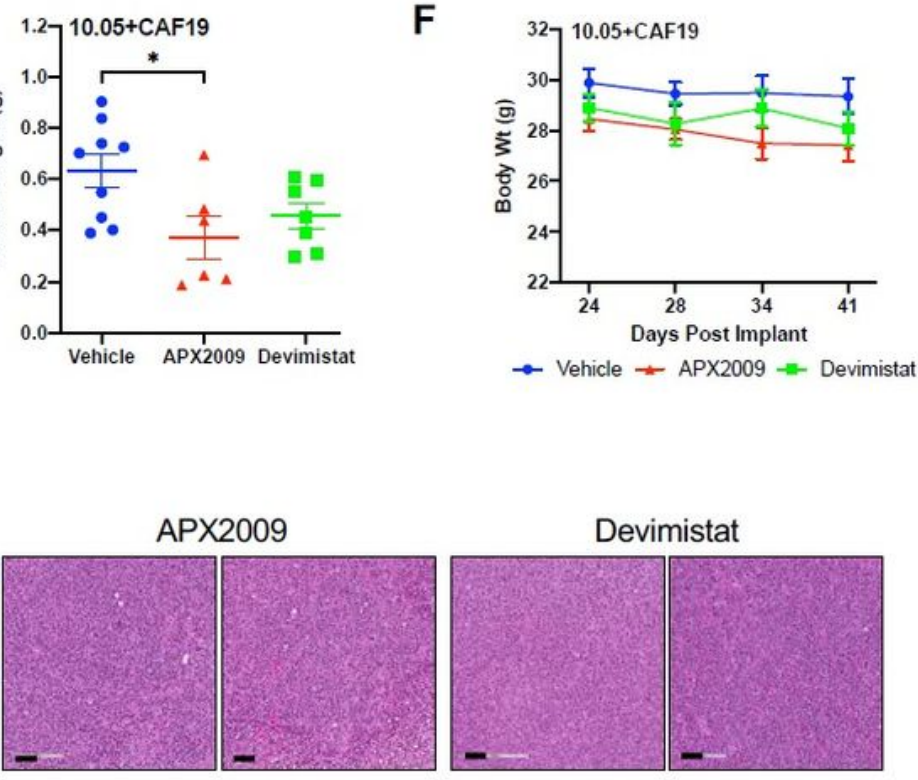

10.05+CAF19
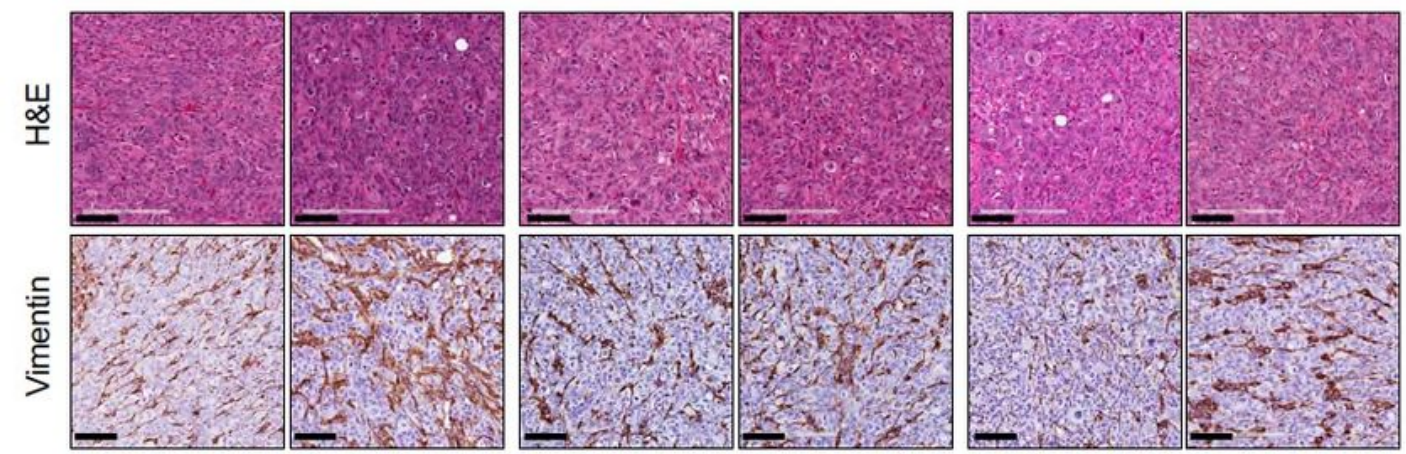

\section{Figure 5}

Ref-1 inhibition with APX2009 results in diminished tumor growth. Tumor growth of Pa03C (A) or Panc10.05+CAF19 (D) subcutaneous xenografts treated with PKT Vehicle or $35 \mathrm{mg} / \mathrm{Kg}$ APX2009 or $50 \mathrm{mg} / \mathrm{Kg}$ Devimistat (\#\#p<0.0001, compared to vehicle control) twice a day, $8 \mathrm{~h}$ apart, continuously for 
15 (for Pa03C xenografts) or 20 (for Panc10.05+CAF19 xenografts) days. And corresponding tumor weights $\left(B, E,{ }^{*} p<0.05\right.$, compared to vehicle control). C\&F. Graphs representing body weights over time for Pa03C or Panc10.05+CAF19 xenografts. Representative images for H\&E and IHC staining for vimentin positivity for Pa03C tumors and Panc10.05+CAF19 (G). All data are represented as Mean \pm SE.
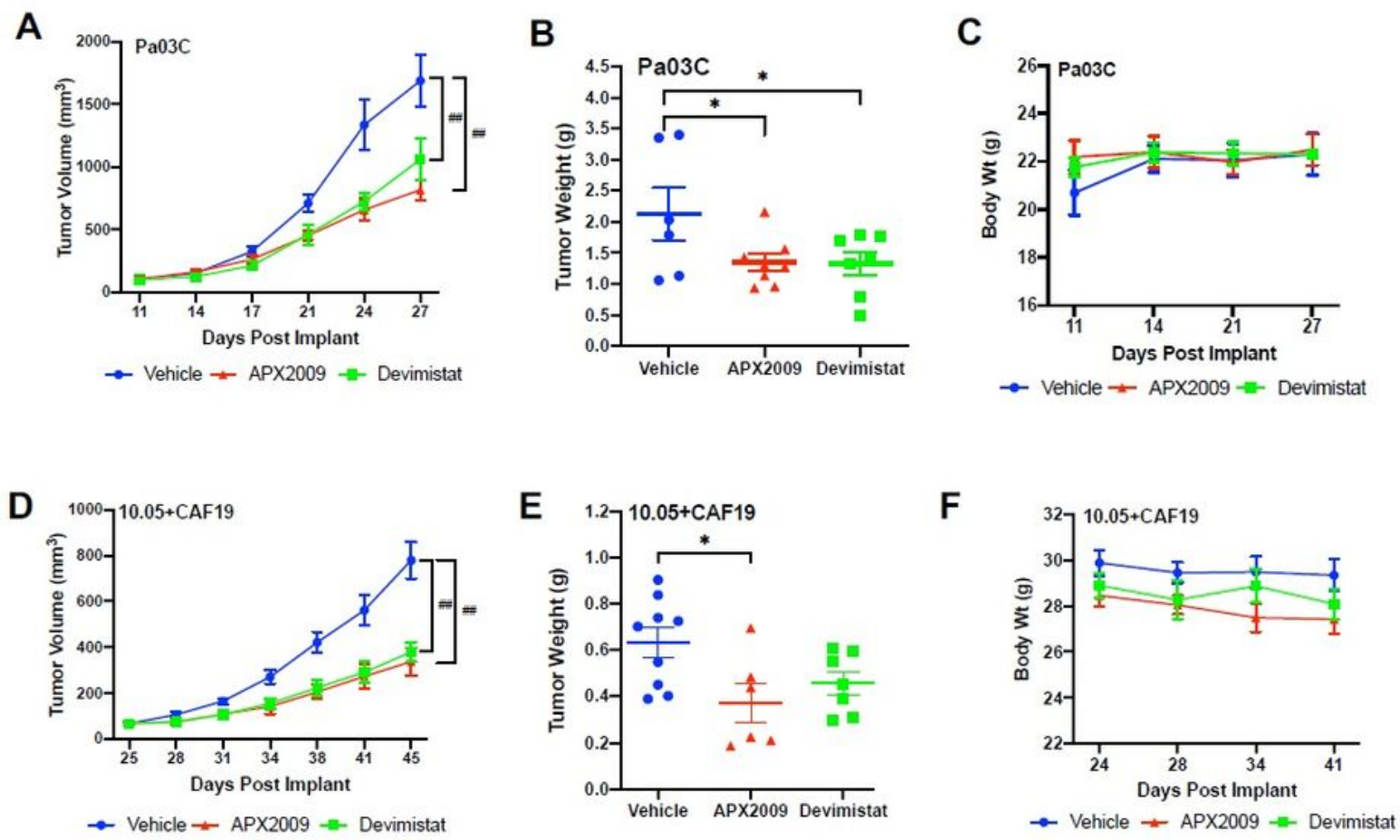

G
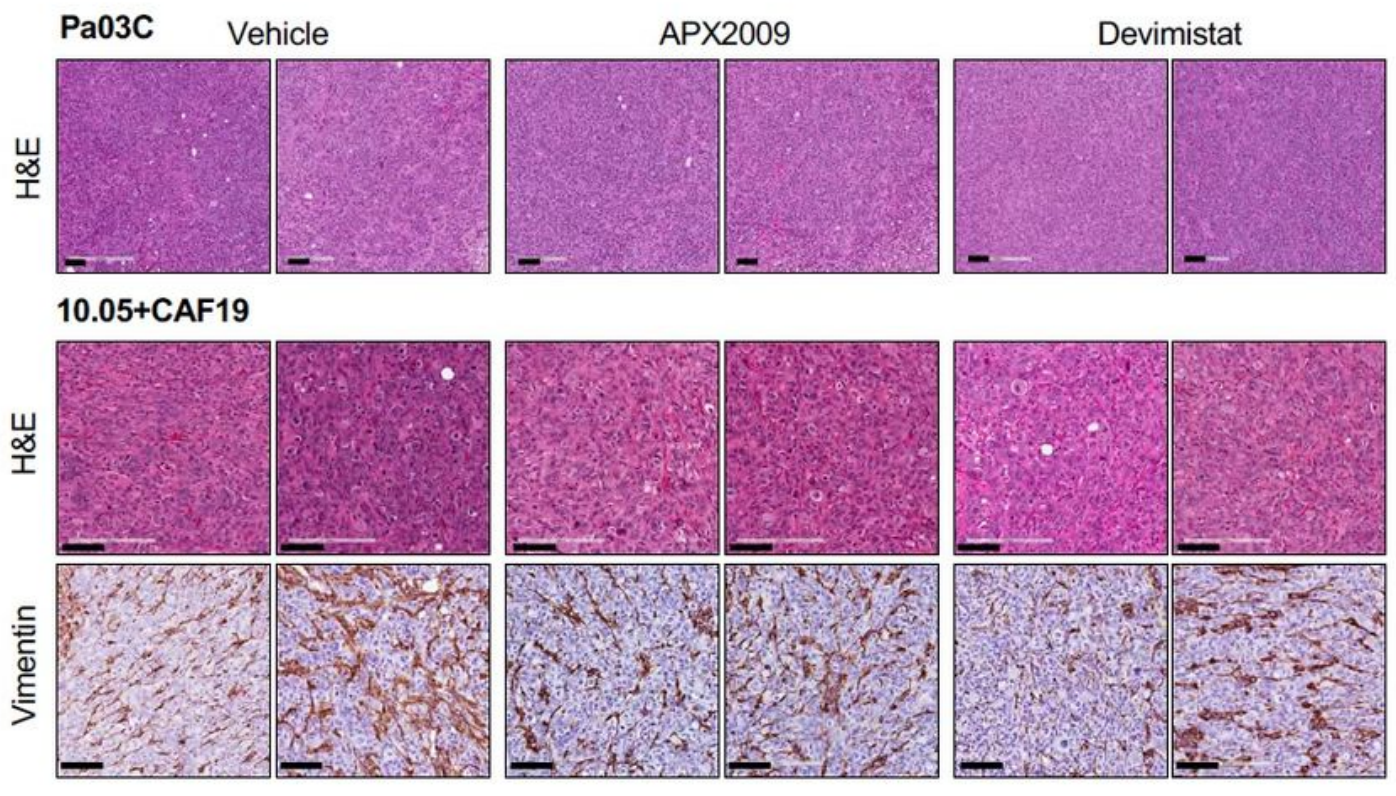

Figure 5 
Ref-1 inhibition with APX2009 results in diminished tumor growth. Tumor growth of Pa03C (A) or Panc10.05+CAF19 (D) subcutaneous xenografts treated with PKT Vehicle or $35 \mathrm{mg} / \mathrm{Kg}$ APX2009 or $50 \mathrm{mg} / \mathrm{Kg}$ Devimistat (\#\#p<0.0001, compared to vehicle control) twice a day, $8 \mathrm{~h}$ apart, continuously for 15 (for Pa03C xenografts) or 20 (for Panc10.05+CAF19 xenografts) days. And corresponding tumor weights $\left(B, E,{ }^{*} p<0.05\right.$, compared to vehicle control). C\&F. Graphs representing body weights over time for $\mathrm{Pa} 03 \mathrm{C}$ or Panc10.05+CAF19 xenografts. Representative images for H\&E and IHC staining for vimentin positivity for Pa03C tumors and Panc10.05+CAF19 (G). All data are represented as Mean \pm SE.

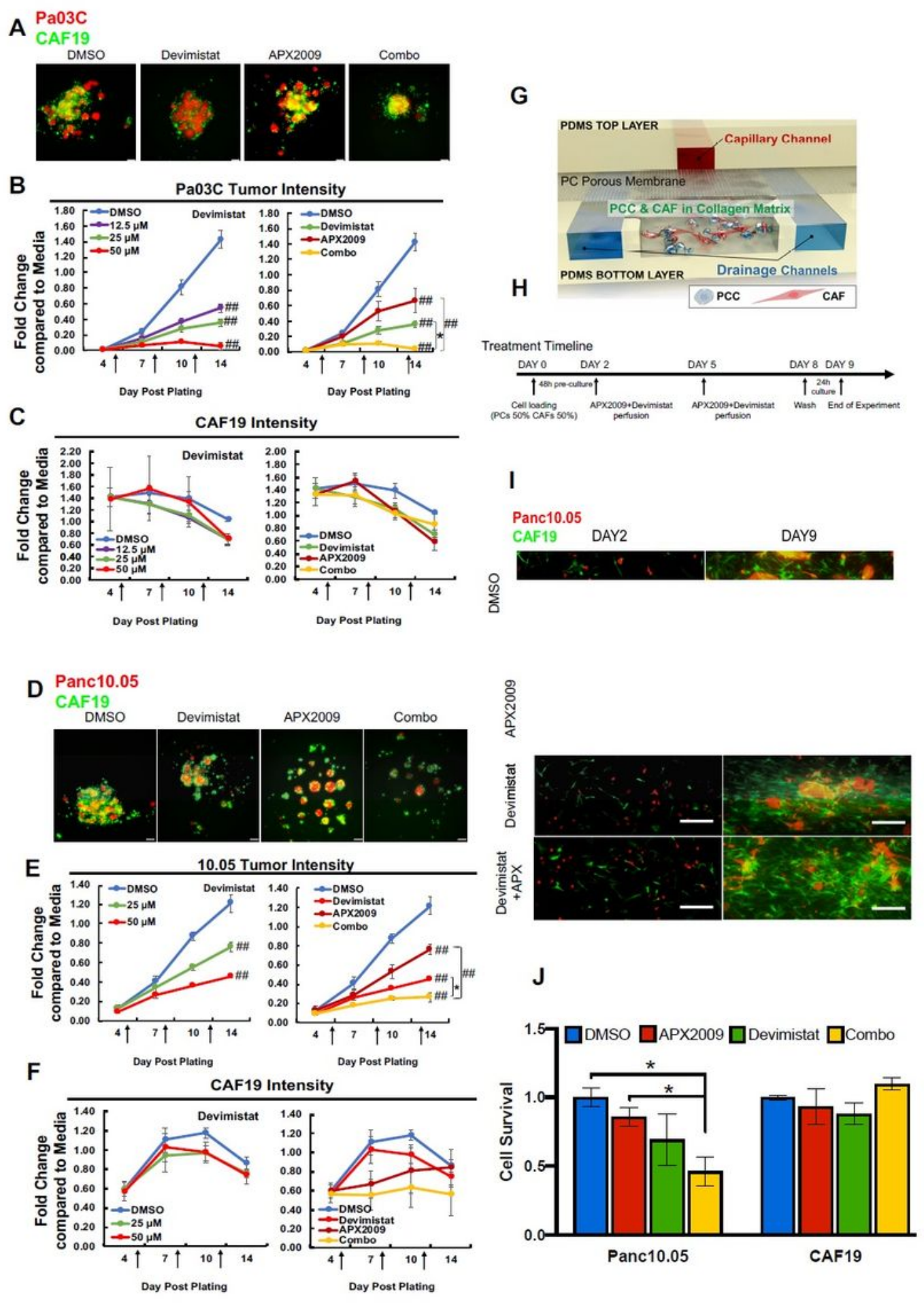




\section{Figure 6}

Ref-1 inhibition in combination with Devimistat attenuates growth in two co-culture models of pancreatic cancer: 3D spheroids and i-TMOC. Representative pictures of two low passage patient-derived low passage cell lines, Pa03C (A) and Panc10.05 (D) plated as 3D co-cultures with CAF19 cells at a ratio of 1:4. These co-cultures were treated with increasing concentrations of Devimistat (0-50 $\mu \mathrm{M})$ and in combination with APX2009 following intensity measurements on Days 4, 7, 10, and 14. For combination

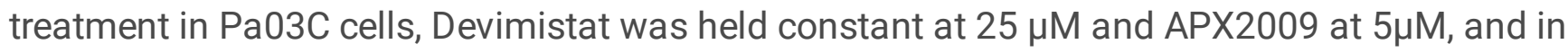
Panc10.05, Devimistat was held constant at $50 \mu \mathrm{M}$ and APX2009 at $10 \mu \mathrm{M}\left({ }^{*} \mathrm{p}<0.05, \# \# 0.0001\right)$. Intensity of the tumor cells (red, B,E) as well as the CAFs (green, C,F) are represented as fluorescence intensity data normalized to Day 14 media control. Graphs are means with standard error of $n=3-4$ and arrows correspond to treatment times. G. Schematic of functional structure of PDAC iT-MOC and experimental timeline in H. I. Fluorescent microscopic observation of Panc10.05 (red) and CAF19 (green) in PDAC iTMOC on Day 2 and Day 9. J. Quantitation of cell survival in the iT-MOC system with single agent (APX2009 $-30 \mu \mathrm{M} /$ Devimistat $-25 \mu \mathrm{M})$ and combination treatment $\left(n \geq 3\right.$, Mean \pm S.E. $\left.{ }^{*} p<0.05\right)$. 


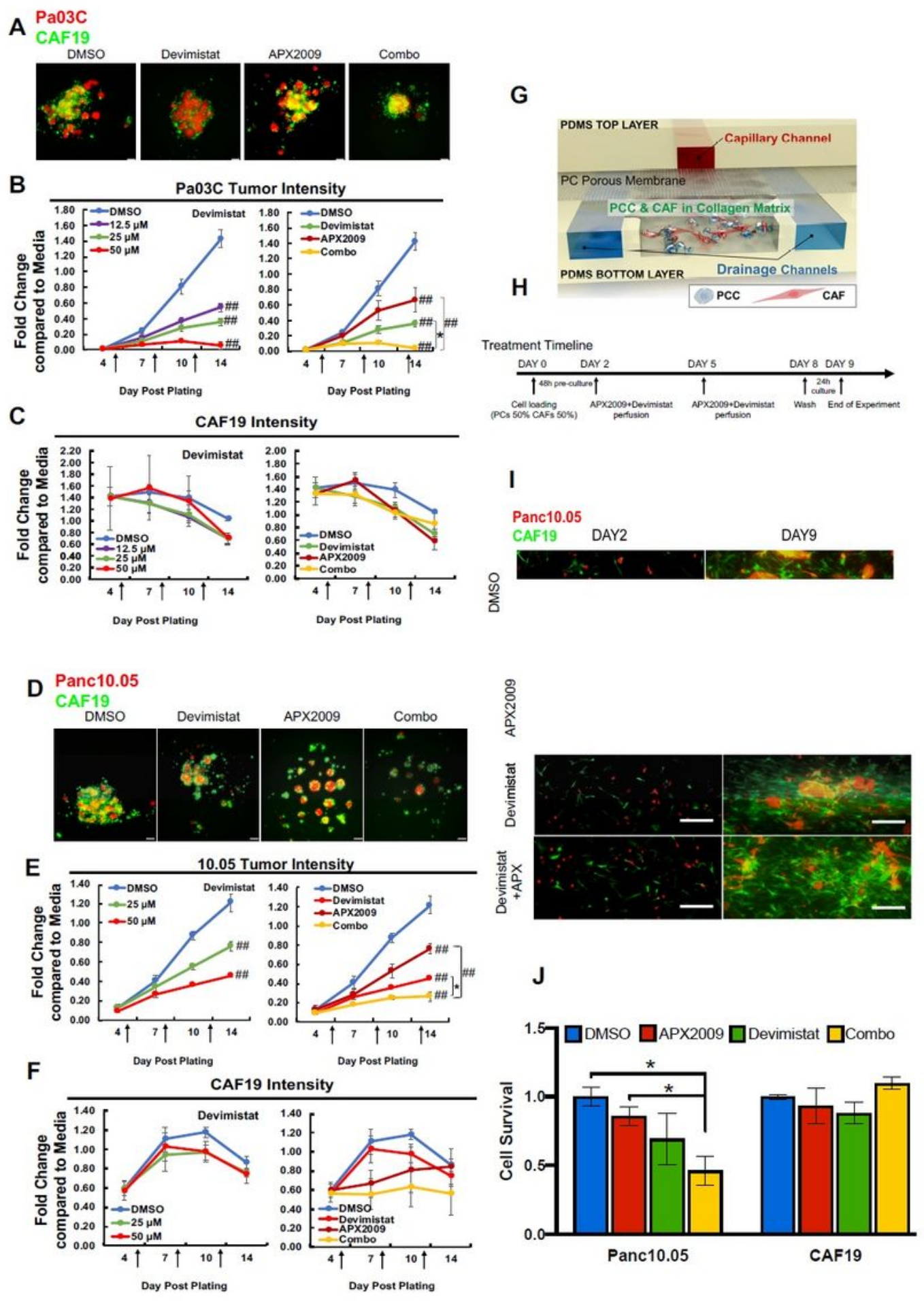

\section{Figure 6}

Ref-1 inhibition in combination with Devimistat attenuates growth in two co-culture models of pancreatic cancer: 3D spheroids and i-TMOC. Representative pictures of two low passage patient-derived low passage cell lines, Pa03C (A) and Panc10.05 (D) plated as 3D co-cultures with CAF19 cells at a ratio of 1:4. These co-cultures were treated with increasing concentrations of Devimistat $(0-50 \mu \mathrm{M})$ and in combination with APX2009 following intensity measurements on Days 4, 7, 10, and 14. For combination 
treatment in Pa03C cells, Devimistat was held constant at $25 \mu \mathrm{M}$ and APX2009 at $5 \mu \mathrm{M}$, and in Panc10.05, Devimistat was held constant at $50 \mu \mathrm{M}$ and APX2009 at $10 \mu \mathrm{M}\left({ }^{*} \mathrm{p}<0.05, \# \# 0.0001\right)$. Intensity of the tumor cells (red, B,E) as well as the CAFs (green, C,F) are represented as fluorescence intensity data normalized to Day 14 media control. Graphs are means with standard error of $n=3-4$ and arrows correspond to treatment times. G. Schematic of functional structure of PDAC IT-MOC and experimental timeline in H. I. Fluorescent microscopic observation of Panc10.05 (red) and CAF19 (green) in PDAC iTMOC on Day 2 and Day 9. J. Quantitation of cell survival in the iT-MOC system with single agent (APX2009 $-30 \mu \mathrm{M} /$ Devimistat $-25 \mu \mathrm{M})$ and combination treatment $\left(n \geq 3\right.$, Mean \pm S.E. $\left.{ }^{*} p<0.05\right)$. 


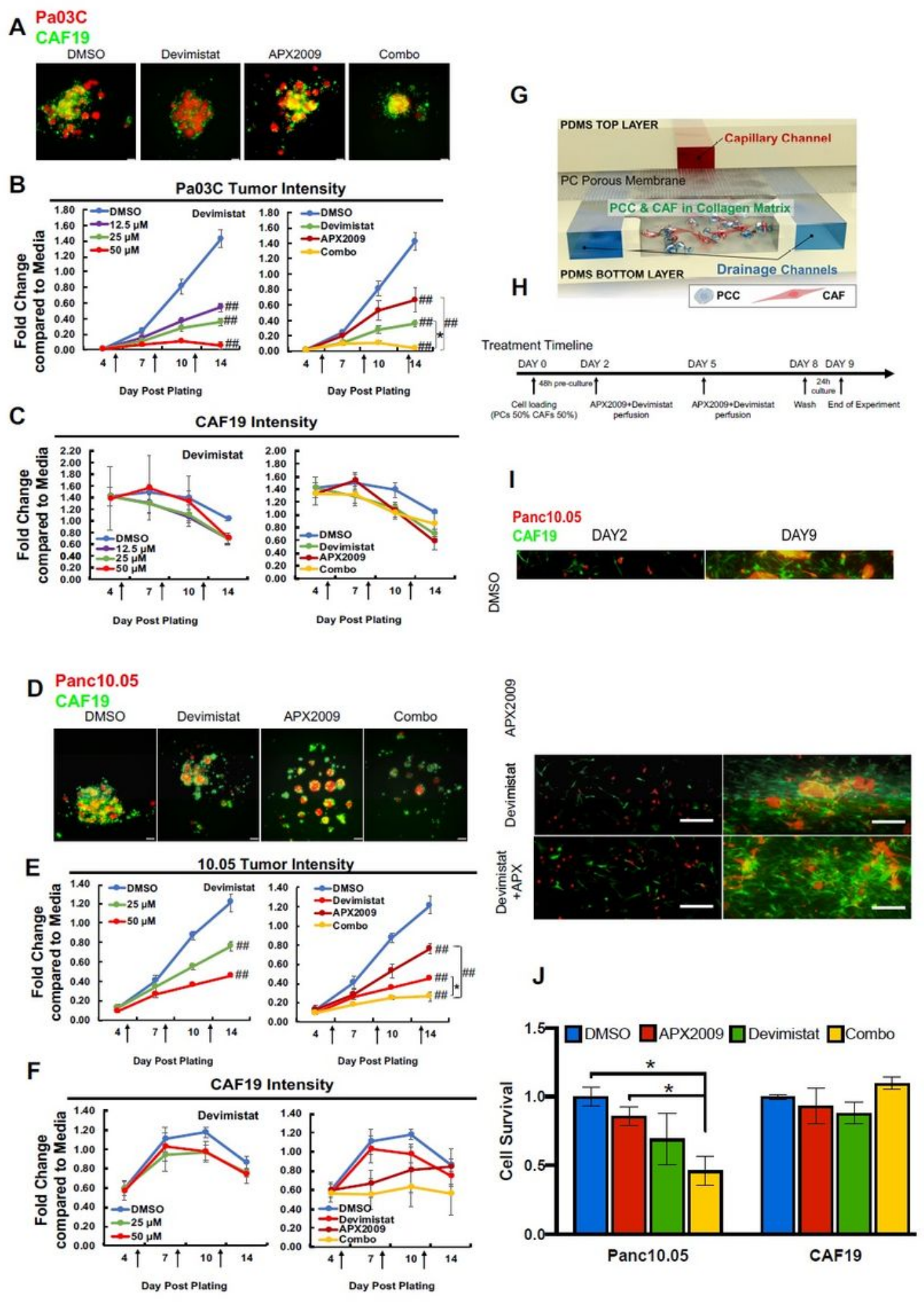

\section{Figure 6}

Ref-1 inhibition in combination with Devimistat attenuates growth in two co-culture models of pancreatic cancer: 3D spheroids and i-TMOC. Representative pictures of two low passage patient-derived low passage cell lines, Pa03C (A) and Panc10.05 (D) plated as 3D co-cultures with CAF19 cells at a ratio of 1:4. These co-cultures were treated with increasing concentrations of Devimistat $(0-50 \mu \mathrm{M})$ and in combination with APX2009 following intensity measurements on Days 4, 7, 10, and 14. For combination 
treatment in Pa03C cells, Devimistat was held constant at $25 \mu \mathrm{M}$ and APX2009 at $5 \mu \mathrm{M}$, and in Panc10.05, Devimistat was held constant at $50 \mu \mathrm{M}$ and APX2009 at $10 \mu \mathrm{M}\left({ }^{*} \mathrm{p}<0.05\right.$, \#\#0.0001). Intensity of the tumor cells (red, B,E) as well as the CAFs (green, C,F) are represented as fluorescence intensity data normalized to Day 14 media control. Graphs are means with standard error of $n=3-4$ and arrows correspond to treatment times. G. Schematic of functional structure of PDAC iT-MOC and experimental timeline in H. I. Fluorescent microscopic observation of Panc10.05 (red) and CAF19 (green) in PDAC iTMOC on Day 2 and Day 9. J. Quantitation of cell survival in the iT-MOC system with single agent (APX2009 $-30 \mu \mathrm{M} /$ Devimistat $-25 \mu \mathrm{M}$ ) and combination treatment $\left(n \geq 3\right.$, Mean \pm S.E. $\left.{ }^{*} p<0.05\right)$.

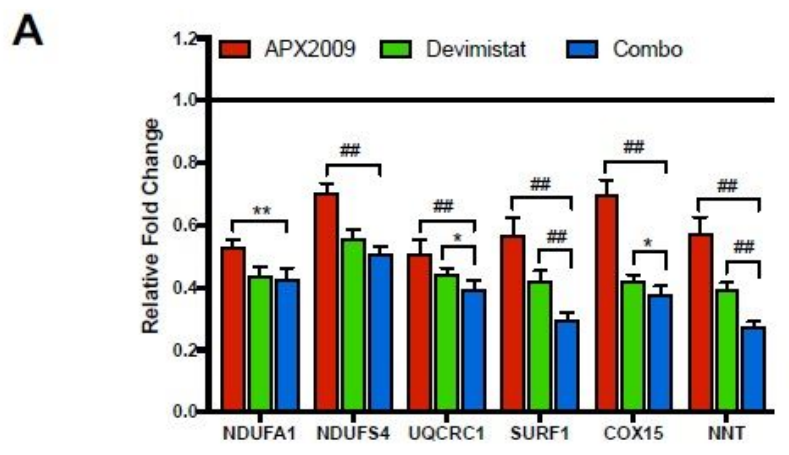

B
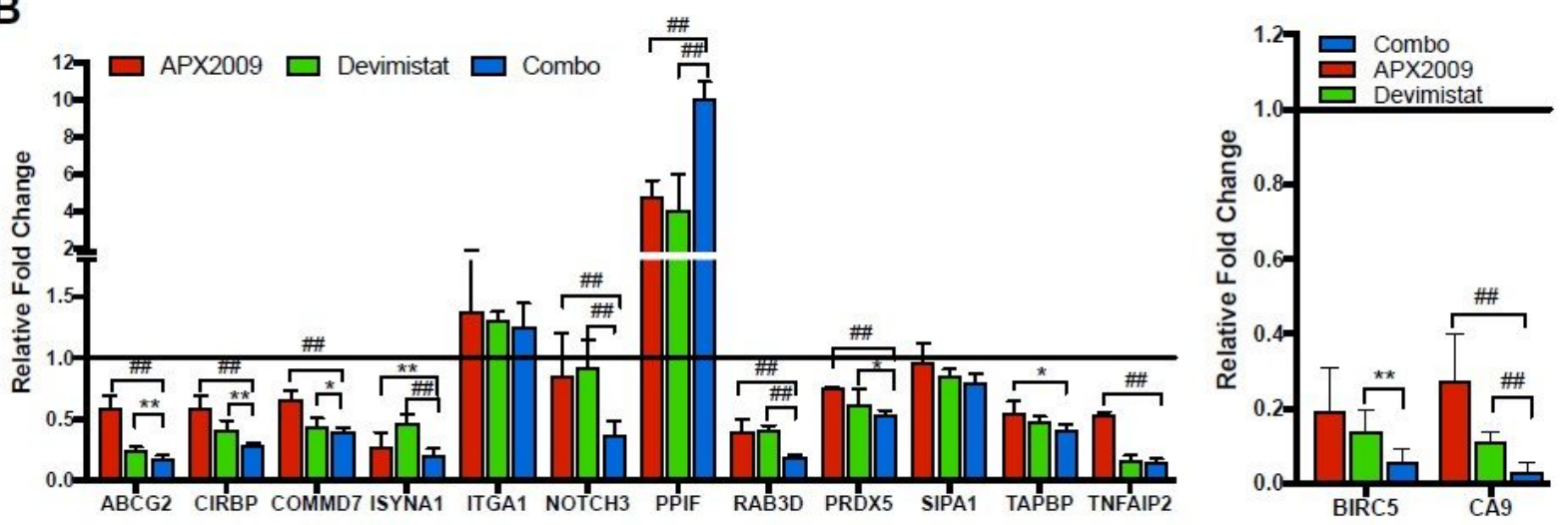

C

D
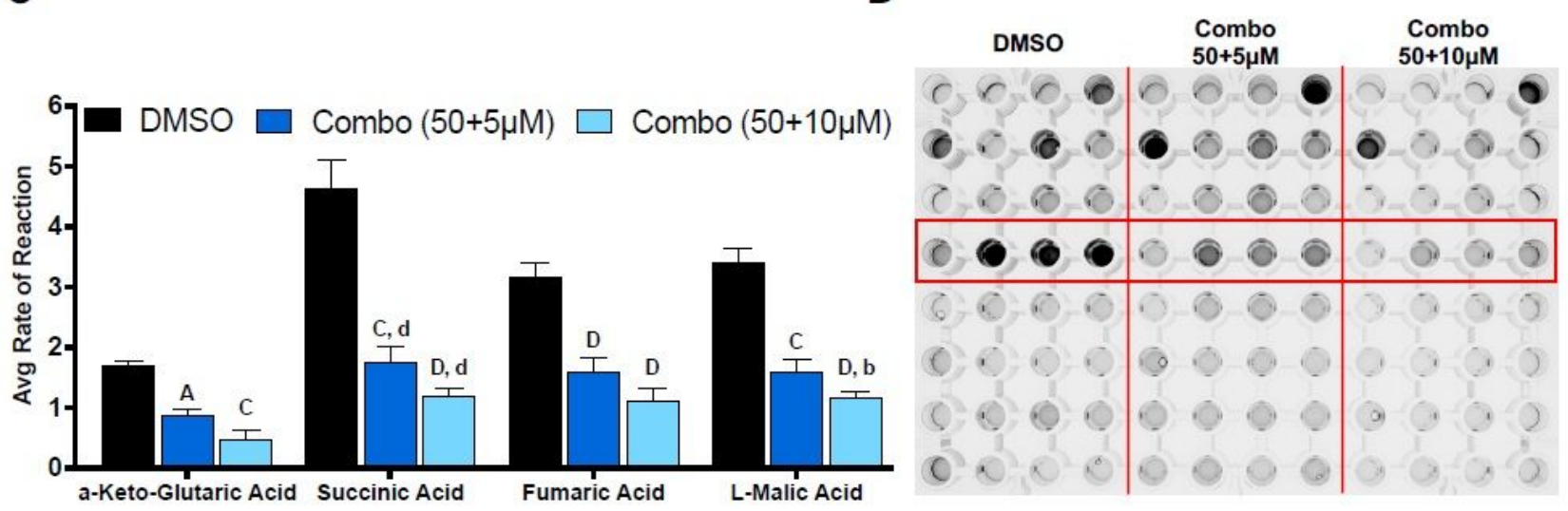


\section{Figure 7}

Ref-1 inhibition in combination with Devimistat downregulates gene expression of the mitochondrial complex genes as well as mitochondrial function. Expression of mitochondrial genes (A) and Ref-1 regulated genes (B) via qPCR in Pa03C 3D spheroids treated with DMSO or a combination of APX2009 (5 $\mu \mathrm{M})$ and Devimistat $(50 \mu \mathrm{M})(n=3, p<0.05-0.0001)$. The data with single agents is also in Figure $3 E$ and $3 \mathrm{~F}$ and provided here for comparison to combination. C. Mitochondrial functional assay in $\mathrm{PaO} \mathrm{C}$ with DMSO or a combination of APX2009 $(5 \& 10 \mu \mathrm{M}, 24 \mathrm{~h})$ and Deviminstat $(50 \mu \mathrm{M}, 24 \mathrm{~h})(\mathrm{n}=3, \mathrm{p}<0.01$, $0.0001)$ and the representative image of the plate following the reaction (D). Data represent Mean $\pm S E$; Uppercase letters denote statistical significance of combination treatment to APX2009 (5 $\mu \mathrm{M})$ and lowercase letters denote statistical significance of combination treatment to Devimistat $(50 \mu \mathrm{M})$. 

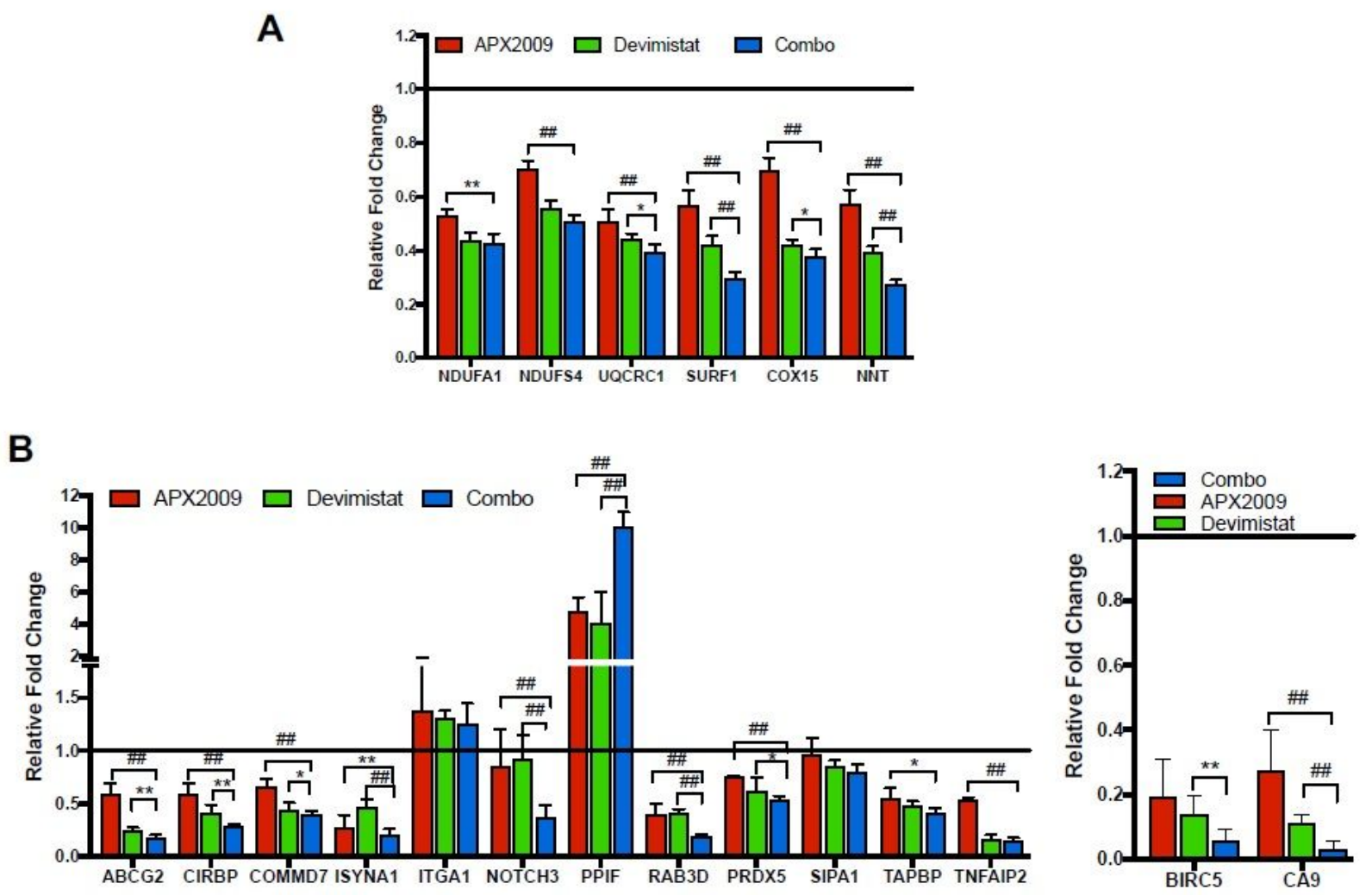

\section{C}

D
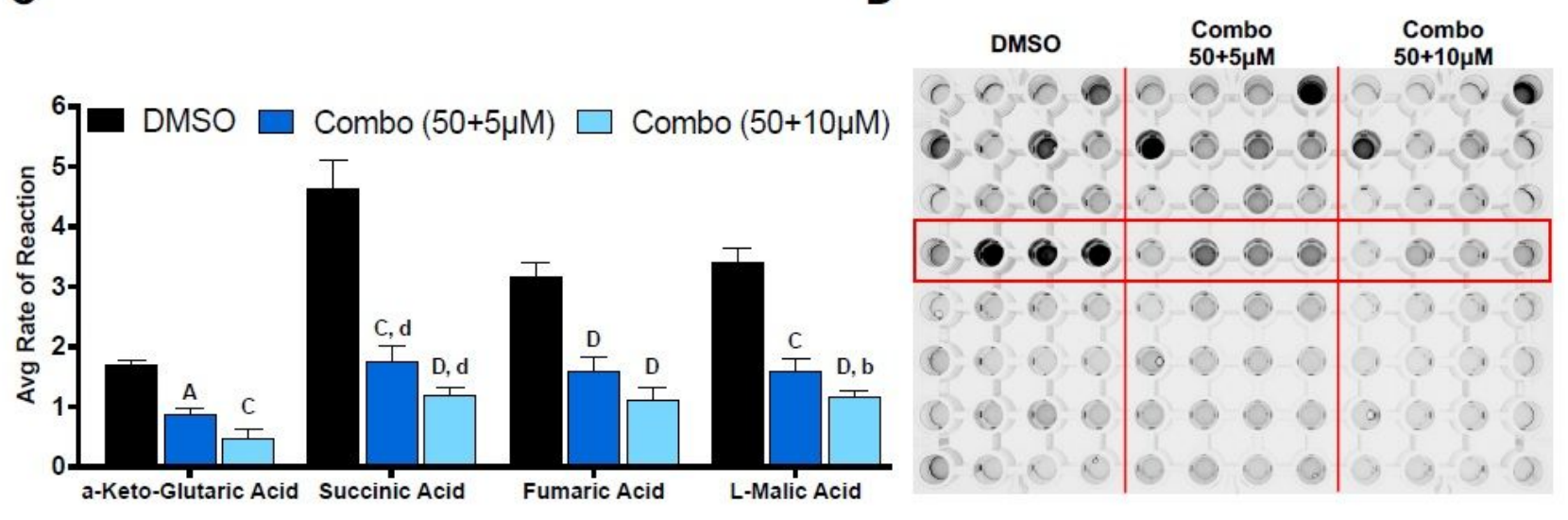

Figure 7

Ref-1 inhibition in combination with Devimistat downregulates gene expression of the mitochondrial complex genes as well as mitochondrial function. Expression of mitochondrial genes (A) and Ref-1 regulated genes (B) via qPCR in Pa03C 3D spheroids treated with DMSO or a combination of APX2009 (5 $\mu M)$ and Devimistat $(50 \mu M)(n=3, p<0.05-0.0001)$. The data with single agents is also in Figure $3 E$ and $3 \mathrm{~F}$ and provided here for comparison to combination. C. Mitochondrial functional assay in $\mathrm{Pa} 03 \mathrm{C}$ with DMSO or a combination of APX2009 $(5 \& 10 \mu \mathrm{M}, 24 \mathrm{~h})$ and Deviminstat $(50 \mu \mathrm{M}, 24 \mathrm{~h})(\mathrm{n}=3, \mathrm{p}<0.01$, 
$0.0001)$ and the representative image of the plate following the reaction (D). Data represent Mean $\pm S E$; Uppercase letters denote statistical significance of combination treatment to APX2009 (5 $\mu \mathrm{M})$ and lowercase letters denote statistical significance of combination treatment to Devimistat $(50 \mu \mathrm{M})$.
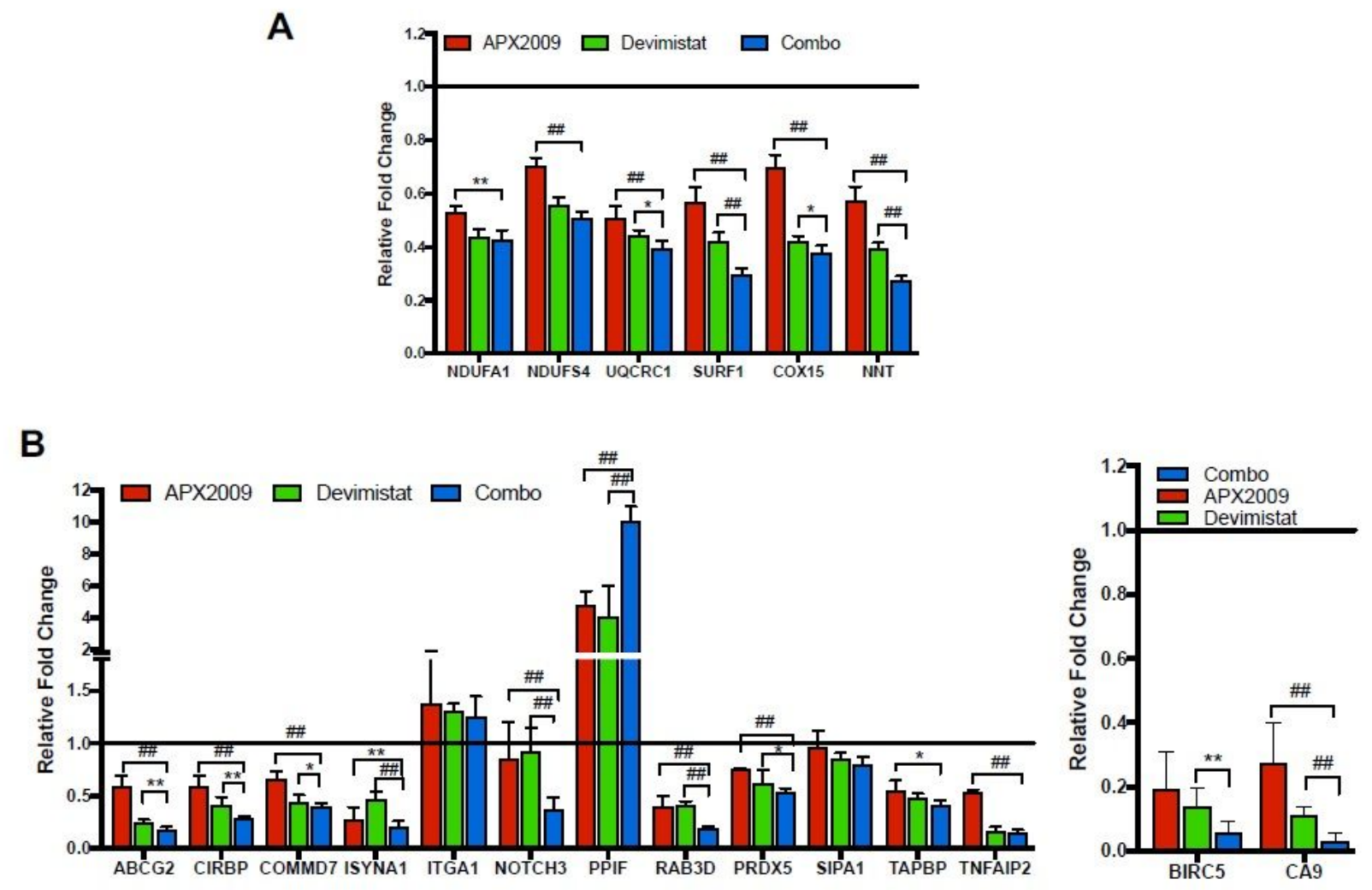

C

D

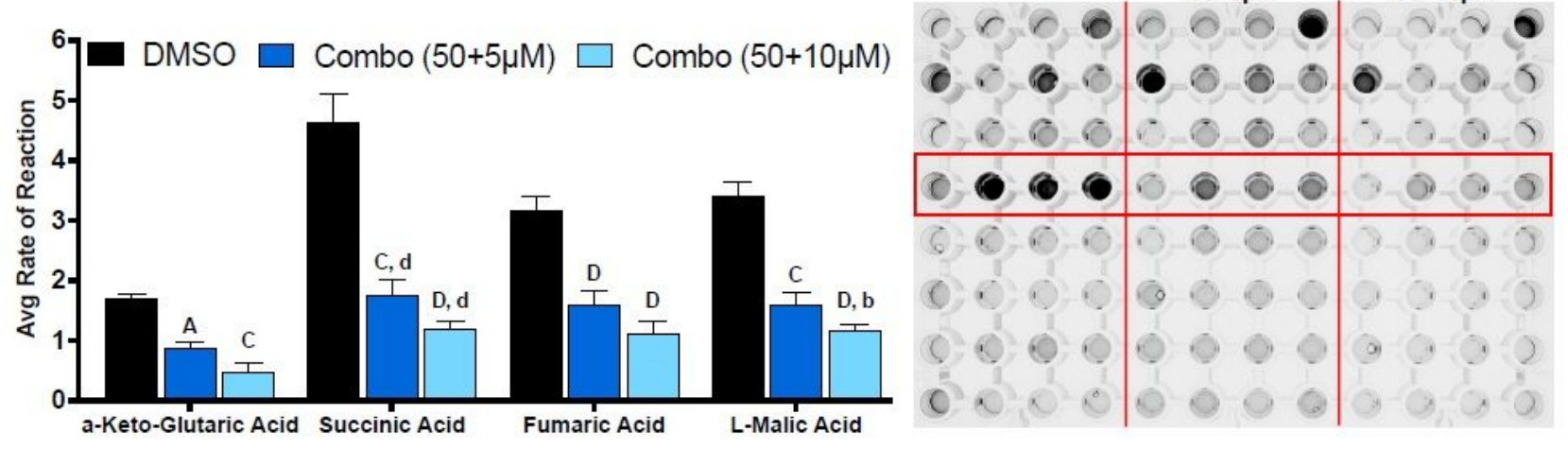

Figure 7

Ref-1 inhibition in combination with Devimistat downregulates gene expression of the mitochondrial complex genes as well as mitochondrial function. Expression of mitochondrial genes (A) and Ref-1 regulated genes (B) via qPCR in Pa03C 3D spheroids treated with DMSO or a combination of APX2009 (5 
$\mu \mathrm{M})$ and Devimistat $(50 \mu \mathrm{M})(\mathrm{n}=3, \mathrm{p}<0.05-0.0001)$. The data with single agents is also in Figure $3 \mathrm{E}$ and $3 \mathrm{~F}$ and provided here for comparison to combination. C. Mitochondrial functional assay in $\mathrm{Pa} 03 \mathrm{C}$ with DMSO or a combination of APX2009 $(5 \& 10 \mu \mathrm{M}, 24 \mathrm{~h})$ and Deviminstat $(50 \mu \mathrm{M}, 24 \mathrm{~h})(\mathrm{n}=3, \mathrm{p}<0.01$, 0.0001 ) and the representative image of the plate following the reaction (D). Data represent Mean $\pm S E$; Uppercase letters denote statistical significance of combination treatment to APX2009 (5 $\mu \mathrm{M})$ and lowercase letters denote statistical significance of combination treatment to Devimistat (50 $\mu \mathrm{M})$.

\section{Supplementary Files}

This is a list of supplementary files associated with this preprint. Click to download.

- SupptableS3DEGtable.xlsx

- SupptableS3DEGtable.xIsx

- SupptableS3DEGtable.xlsx

- SupptableS4PE.xIsx

- SupptableS4PE.xIsx

- SupptableS4PE.xIsx

- Supptables5coregulationmoduleinfo.csv

- Supptables5coregulationmoduleinfo.csv

- Supptables5coregulationmoduleinfo.csv

- SupplementalFigureLegends.docx

- SupplementalFigureLegends.docx

- SupplementalFigureLegends.docx

- SupplementalFigures112820.pptx

- SupplementalFigures112820.pptx

- SupplementalFigures112820.pptx

- SupplementalTables112820.pdf

- SupplementalTables112820.pdf

- SupplementalTables112820.pdf

- Supplementalinformation112820.docx

- Supplementalinformation112820.docx

- Supplementalinformation112820.docx 